\title{
Iterates of differential operators and vector valued functions on non quasi analytic classes
}
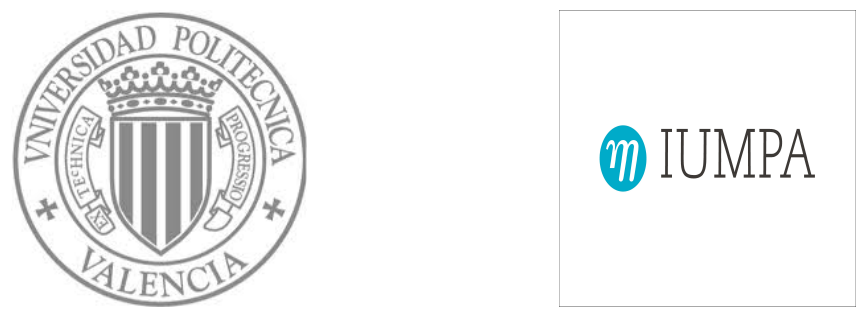

Jordi Juan Huguet

jorjuahu@upvnet.upv.es

Institut Universitari de Matemàtica Pura i Aplicada.

Departament de Matemàtica Aplicada.

Universidad Politècnica de València.

Camí de Vera S/N. 46022 València.

Memoria presentada por Jordi Juan Huguet para optar al grado de Doctor. Trabajo dirigido por los Dres.

Don José Bonet Solves y Don Antonio Galbis Verdú.

Valencia, 2011 

Don José Bonet Solves, Catedrático de Universidad en el Departamento de Matemática Aplicada de la Universidad Politécnica de Valencia y Don Antonio Galbis Verdú, Profesor Titular de Universidad en el Departamento de Análisis Matemático de la Universidad de Valencia

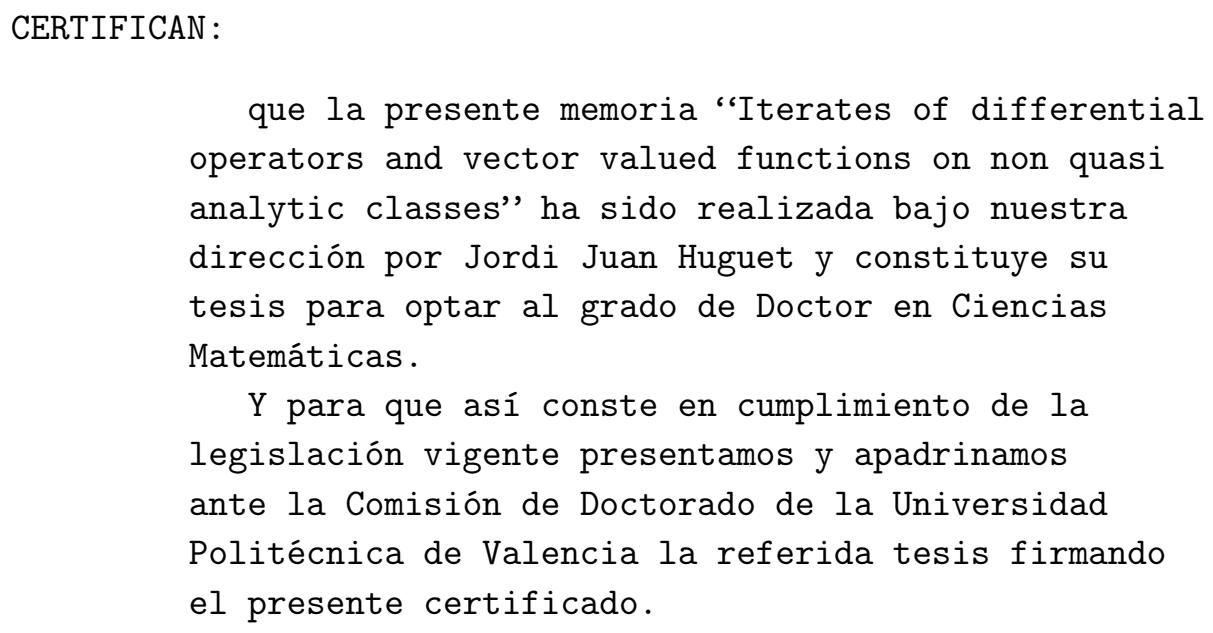

Valencia, diciembre 2010

Los directores:

José Bonet Solves y Antonio Galbis Verdú 



\section{Agraïments}

Des que estava cursant els primers anys de la Llicenciatura en Matemàtiques he tingut la il.lusió de fer una tesi doctoral en aquesta disciplina, com deia Gauss, la reina de les ciències. Segurament aquesta il.lusió ha sorgit de la curiositat que les Matemàtiques i la ciència han despertat sempre en mi i pel fet que durant els meus anys com a estudiant vaig tindre el privilegi de poder contemplar el procés de creació matemàtica al despatx del professor Adolfo Ballester, quan ell investigava amb els membres del seu grup de Teoria de Grups, junt a destacats investigadors estrangers. Sembla que aquesta il.lusió, després d'aquests anys d'aprenentatge, esforç, interessants reptes $i$ algunes nits sense dormir, serà prompte una realitat. Crec que tinc la sort de poder dir que, si el temps tornara enrere, decidiria de nou fer una tesi doctoral en Matemàtiques. Per tant, sent que aquest viatge ha valgut la pena. Sense dubte aquest fet ha sigut gràcies a certes persones que m'han ajudat d'una manera o d'una altra; per això, ara és un bon moment per a mostrar-los els meus més que sincers agraïments.

En primer lloc, vull donar les gràcies a dos matemàtics a qui admire $i$ estime. Són els meus directors de tesi: els professors Pepe Bonet i Antonio Galbis. Agraïsc el temps que han dedicat a la meua formació, que hagen confiat en mi $i$ que m'hagen donat l'oportunitat de doctorar-me $i$ aprendre tantes coses. Sense la seua ajuda i la seua direcció, aquest treball no hauria vist mai la llum i, menys encara, hauria esdevingut en un seguit d'articles d'investigació. He gaudit dels temes de recerca que han sel-leccionat, que, a la vegada, m'han permés conéixer diverses línies $i$ tendències de l'Anàlisi Funcional.

El quart capitol d'aquesta tesi presenta els resultats d'un article conjunt amb el meu director Antonio Galbis i la professora Carmina Fernández sobre espais de Fréchet d'ultradistribucions invariants per derivació. Vull agrair la generositat de Carmina Fernández, qui em va animar a participar en aquesta col.laboració quan estàvem a Poznan (Polònia). Els seus consells $i$ suggeriments han estat sempre molt encertats.

I am very indebted to Professors Leonhard Frerick and Jochen Wengenroth for their help, advice, suggestions and inspiring discussions during my stay in the University of Trier in autumn 2009. Definitely, those three months in Trier 
were a very interesting experience. It would be a pleasure to visit their department again: there is a fantastic atmosphere to work and to learn mathematics.

La ricerca del professore Luigi Rodino sugli iterati di operatori ha motivato una parte importante di questa tesi. Grazie mille per accettare la mia visita al Dipartimento di Matematica all' Università di Torino. Purtroppo non ho potuto realizzare questa visita perche ho ottenuto un posto all'Universitat de Valencia. Spero di fare questa visita prossimamente.

El meus amics i germans científics, David Jornet i Alberto Conejero, sempre han exercit com a tals: com a germans majors acadèmicament parlant. Les seues recomanacions $i$ recolzament han sigut molt importants per a mi des de l'inici d'aquest treball.

Un agraïment molt especial a Adolfo Ballester, pel que he dit abans i per moltres altres coses. Ell va ser qui em va recomanar el meu actual grup d'investigació, un dels millors consells que m'han donat.

Per últim vull mencionar la meua família, perquè m'ha ajudat a deixar l'abstracció $i$ a xafar terra. Sobretot vull destacar mon pare i ma mare, que m'han animat sempre a seguir amb els meus projectes $i$ han creat a casa un ambient on l'estudi i la cultura són essencials. Gràcies a ells he pogut créixer entre llibres. Un record per al meus germans, Miquel i Josep, i per a Jim, a qui va dedicada aquesta tesi. 
A Jim, tot un senyor. 
VI

Esta memoria ha sido elaborada en el Instituto Universitario de Matemática Pura y Aplicada de la Universidad Politécnica de Valencia durante el período de disfrute de una Beca de Predoctoral (referencia AP-200604678) del Programa Nacional de Formación de Profesorado Universitario del Ministerio de Educación y Ciencia. Una ayuda complementaria de dicho programa ha permitido al autor realizar una estancia de investigación en el Departamento de Matemáticas de la Universidad de Trier, Alemania (septiembre-diciembre de 2009) bajo la dirección del profesor Leonhard Frerick.

Esta investigación también ha sido financiada parcialmente por los proyectos: "Espacios de Fréchet, Operadores de Convolución y Operadores Hipercíclicos" (MTM2004-02262, MEC y FEDER), "Métodos de análisis funcional para el análisis complejo y las ecuaciones en derivadas parciales" (MTM2007-62643, MEC y FEDER), “Operadores de extensión de Whitney, curvas ultradiferenciables, iterados de operadores diferenciales y frente de ondas " (Project GV/2010/040, Conselleria d'Educació de la GVA) y la red "Variable Compleja, espacios de funciones y operadores entre ellos" (MTM2006-26627-E). 




\section{Contents}

Resum $\quad x$

Resumen $\quad$ XIII

Summary $\quad$ XV

Introduction and motivation $\quad$ XVII

1. Preliminaries: non-quasianalytic classes 1

2. Generalized non-quasianalytic classes $\quad 11$

2.1. The class $\mathcal{E}_{P, \omega}(\Omega) \ldots \ldots \ldots \ldots \ldots \ldots$

2.2. The completeness of $\mathcal{E}_{P, \omega}(\Omega) \ldots \ldots \ldots \ldots$

2.3. A Paley-Wiener type theorem and its consequences . . . . . 29

3. The problem of iterates on non-quasianalytic classes 45

3.1. The growth of $\mathcal{E}_{P, \omega}(\Omega) \ldots \ldots \ldots \ldots \ldots$

3.2. Results on the problem of iterates . . . . . . . . . . 55

4. Fréchet spaces invariant under differentiation 59

4.1. Preliminaries: ultradifferential operators . . . . . . . . . . 60

4.2. Fréchet spaces invariant under ultradifferential operators . . 61

4.3. Iterates and Fréchet spaces invariant under partial differential operators . . . . . . . . . . . . . . . . 65

5. Vector valued ultradifferentiable functions of Roumieu type in $\begin{array}{ll}\text { Fréchet spaces } & 77\end{array}$

5.1. Definitions and properties . . . . . . . . . . . 77

5.2. Main results . . . . . . . . . . . . . . . . . . 84

$\begin{array}{ll}\text { References } & 86\end{array}$ 



\section{Resum}

L'any 1960 Komatsu va introduir certes classes de funcions $\mathcal{C}^{\infty}$ definides per estimacions del creixement dels successius iterats d'un operador en derivades parcials quan estudiava propietats de regularitat de certes equacions en derivades parcials. Aquesta línia d'investigació ha estat molt activa fins a l'actualitat amb els treballs de molts autors: destaquem, entre altres, Bolley, Camus, Kotake, Langenbruch, Métivier, Narasimhan, Newberger, Rodino, Zanghirati i Zielezny. Tota aquesta bibliografia està relacionada amb l'anomenat problema dels iterats que consisteix, parlant sense presició, en caracteritzar les funcions d'una certa classe mitjançant el comportament dels iterats d'un operador prèviament donat.

En la primera part d'aquesta tesi continuem la investigació descrita anteriorment en un context més general: classes no quasi analítiques segons la teoria de Braun, Meise i Taylor. L'estudi d'aquestes classes és actualment una àrea d'investigació molt activa per les seues aplicacions a la teoria d'operadors diferencials en derivades parcials: destaquem entre altres el treball de Bonet, Braun, Domański, Fernández, Frerick, Galbis, Taylor i Vogt. En el Capítol 1 introduïm aquestes classes i enunciem les propietats que utilitzarem al llarg d'aquesta tesi.

En el Capítol 2 definim les classes no quasi analítiques respecte dels iterats d'un operador en derivades parcials $P(D)$ i estudiem les seues propietats topològiques com la completesa i la nuclearitat. En particular, demostrem que estes classes són un espai localment convex complet si i sols si $P(D)$ és hipoel-líptic i veiem que en aquest cas són també un espai nuclear. Tot seguit, provem per aquestes classes un teorema de tipus PaleyWiener.

El nostre objectiu en el Capítol 3 és donar resultats sobre el problema dels iterats en classes no quasi analítiques. Així, generalitzem resultats de Newberger, Zielezny, Métivier i Komatsu i donem caracteritzacions perquè una classe no quasi analítica definida pels iterats d'un operador coincidesca amb una classe no quasi analítica en el sentit de Braun, Meise i Taylor. 
Tota la investigació feta sobre espais de funcions definits per iterats d'operadors s'havia centrat en classes de tipus Roumieu. Tanmateix, demostrem que els resultats dels capítols 2 i 3 també són certs per a classes de tipus Beurling.

En l'any 1990, Langenbruch i Voigt demostraren que tot espai de Fréchet format per distribucions invariant sota l'acció d'un operador hipoel-líptic està inclòs contínuament en $\mathcal{C}^{\infty}$. En el capítol 4 introduïm els operadors ultradiferencials i investiguem extensions del resultat de Langenbruch i Voigt al context ultradiferenciable. El nou concepte d'espai de Fréchet $(\omega, P(D))$-estable imposa als iterats de l'operador $P(D)$ una condició d'equicontinuitat i ens permet mostrar la relació d'aquest tipus de resultats amb el problema dels iterats.

La segona part d'aquesta tesi se centra en l'estudi de funcions vectorials en un espai localment convex. Motivats per les investigacions anteriors de Bonet, Domański, Komatsu, Kriegl, Michor, Rainer i Schwartz, en el Capítol 5 donem diverses definicions de funció ultradiferenciable amb valors vectorials i demostrem que tota funció dèbilment ultradiferenciable en valors en un espai de Fréchet $E$ és fortament ultradiferenciable si i sols si $E$ té la propietat (DN) de Vogt. D'aquesta manera, resolem un problema proposat per Kriegl i Michor en un congrés que va tindre lloc a Paderborn (Alemanya) en novembre de l'any 2008. 


\section{Resumen}

En el año 1960 Komatsu introdujo ciertas clases de funciones $\mathcal{C}^{\infty}$ definidas mediante estimaciones del crecimiento de los sucesivos iterados de un operador en derivadas parciales cuando estudiaba propiedades de regularidad de las soluciones de ciertas ecuaciones en derivadas parciales. Esta línea de investigación ha sido muy activa hasta la actualidad a través de los trabajos de muchos autores. Destacamos, entre otros, Bolley, Camus, Kotake, Langenbruch, Métivier, Narasimhan, Newberger, Rodino, Zanghirati y Zielezny. Toda esta bibliografía involucra el llamado problema de los iterados que consiste, grosso modo, en caracterizar las funciones de una cierta clase en términos del comportamiento de los iterados de un operador previamente fijado.

En la primera parte de esta tesis seguimos con la investigación mencionada antes en un contexto más general: clases no casi analíticas de funciones ultradiferenciables en el sentido de Braun, Meise y Taylor. El estudio de estas clases no casi analíticas es una área de investigación muy activa debido a sus aplicaciones a la teoría de operadores en derivadas parciales: destacamos entre otros el trabajo de Bonet, Braun, Domański, Fernández, Frerick, Galbis, Taylor y Vogt. En el Capítulo 1 introducimos estas clases y enunciamos las propiedados que utilizaremos a lo largo de esta tesis.

En el Capítulo 2 definimos las clases no casi analíticas con respecto a los iterados de un operador en derivadas parciales $P(D)$ y estudiamos sus propiedades topológicas como la completitud y la nuclearidad. En particular, demostramos que estas clases son un espacio localmente convexo completo si y sólo si el operador $P(D)$ es hipoelíptico y vemos que en tal caso son además un espacio nuclear. A continuación, demostramos que estas clases verifican un teorema de tipo Paley-Wiener.

En el Capítulo 3 tenemos como objetivo obtener resultados sobre el problema de los iterados en clases no casi analíticas. Generalizamos varios resultados de Newberger, Zielezny, Métivier y Komatsu y damos caracterizaciones de cuándo una clase no casi analítica definida en términos de 
los iterados de un operador coincide con una clase no casi analítica según Braun, Meise y Taylor.

Toda la investigación que se había hecho sobre espacios de funciones definidos por iterados de operadores se había centrado en clases de tipo Roumieu. Sin embargo, demostramos que los resultados dados en los Capítulos 2 y 3 también son válidos para clases de tipo Beurling.

En el año 1990, Langenbruch y Voigt demostraron que todo espacio de Fréchet formado por distribuciones que sea invariante bajo la acción de un operador hipoelíptico está continuamente incluido en $\mathcal{C}^{\infty}$. En el capítulo 4 introducimos los operadores ultradiferenciales e investigamos extensiones del resultado de Langenbruch y Voigt al contexto ultradiferenciable. El nuevo concepto de espacio de Fréchet $(\omega, P(D))$-estable involucra a los iterados de $P(D)$ mediante una condición de equicontinuidad y nos permite mostrar la relación de este tipo de resultados con el problema de los iterados.

La segunda parte de esta tesis se centra en el estudio de funciones con valores vectoriales en un espacio localmente convexo. Motivados por investigaciones previas de Bonet, Domański, Komatsu, Kriegl, Michor, Rainer y Schwartz, en el Capítulo 5 damos varias definiciones de función ultradiferenciable con valores vectoriales y demostramos que cada función débilmente ultradiferenciable con valores en un espacio de Fréchet $E$ es fuertemente ultradiferenciable si y sólo si $E$ tiene la propiedad (DN) de Vogt. De este modo, resolvemos un problema propuesto por Kriegl y Michor en un congreso celebrado en Paderborn (Alemania) en noviembre del año 2008. 


\section{Summary}

Classes of smooth functions defined by estimates on the growth of the successive iterates of a partial differential operator were introduced by Komatsu in order to study regularity properties of certain partial differential equations. This research was continued until recent days by several authors like Bolley, Camus, Kotake, Langenbruch, Métivier, Narasimhan, Newberger, Rodino, Zanghirati, Zielezny and others. All this work is related to the problem of iterates which consists, roughly speaking, in characterizing the functions in a given class of functions in terms of the behavior of the iterates of a fixed differential operator.

In the first part of this thesis we continue the research described above in a more general setting: non quasi analytic classes of ultradifferentiable functions as defined by Braun, Meise and Taylor. The study of these non quasianalytic classes of ultradifferentiable functions has received much attention in recent years due to its applications to the theory of partial differential operators: we refer to the work of Bonet, Braun, Domański, Fernández, Frerick, Galbis, Taylor and Vogt. In Chapter 1 we introduce these classes and give the properties that will be used throughout this memoir.

In the second chapter we define non quasianalytic classes with respect to the iterates of a partial differential operator $P(D)$ and study their locally convex properties like completeness and nuclearity. In fact, we prove that theses classes are a complete locally convex space if and only if the operator $P(D)$ is hypoelliptic and that, in this case, such spaces are nuclear. After that, it is proved that these classes verify a Paley-Wiener type theorem.

The aim of Chapter 3 is to obtain results concerning the problem of iterates on non quasianalytic classes. We extend results by Newberger, Zielezny, Métivier and Komatsu and give characterizations in order that a non quasianalytic class defined with respect to the iterates of a partial differential operator coincides with a class of ultradifferentiable functions in the sense of Braun, Meise and Taylor.

We want to emphasize that the previous literature on iterates of diffe- 
rential operators only deals with Roumieu classes. However, all the results presented in Chapters 2 and 3 remain true in the Beurling case.

In 1990, Langenbruch and Voigt proved that a Fréchet space of distributions which is stable under a single hypoelliptic differential operator is continuously included in $\mathcal{C}^{\infty}$. In Chapter 4 we introduce ultradifferential operators and give extensions of the result of Langenbruch and Voigt to the ultradifferential setting. The new notion of $(\omega, P(D))$-stable Fréchet space imposes an equicontinuity condition on the successive iterates of $P(D)$ and allows us to show the connections of this topic with the problem of iterates.

In the second part of this thesis we investigate vector valued functions in a locally convex space. Motivated by previous work by Bonet, Domański, Komatsu, Kriegl, Michor, Rainer and Schwartz, we introduce in Chapter 5 several notions of vector valued ultradifferentiable function and we prove that every weakly non-quasianalytic ultradifferentiable function with values in a Fréchet space $E$ is topologically (or strongly) ultradifferentiable if and only if the space $E$ satisfies the topological invariant (DN) of Vogt. Thus, we solve a problem posed by Kriegl and Michor in a meeting celebrated in Paderborn (Germany) in November 2008. 


\section{Introduction and motivation}

The aim of this thesis is to investigate some topics related to non quasianalytic classes. This research is concerned with the theory of functional analysis and more particularly, with the area of Fréchet spaces and its applications to linear partial differential operators. The main purpose of this dissertation is to present some results on:

- Spaces defined in terms of the successive iterates of a differential operator

- Fréchet spaces invariant under differential operators

- Vector valued functions

in the setting of non quasianalytic classes.

The study of several non quasianalytic classes of ultradifferentiable functions, their topological duals (ultradistributions) and convolution operators and linear partial differential operators between these classes has been a very active area of research since the last century. In 1908 Holmgren, when studying regularity properties of solutions of the homogeneous heat equation, introduced certain non quasi analytic classes. More general classes, the so called Gevrey classes, were introduced in 1918 by Gevrey [30]. In the 1960's Roumieu extended the Schwartz's theory of distributions using non quasianalytic classes of ultradifferentiable functions as test spaces. Non quasianalytic classes of ultradifferentiable functions are intermediate classes between real analytic functions and the class of all $\mathcal{C}^{\infty}$-functions containing non trivial elements with compact support. There are essentially two ways to introduce these classes: the theory of Carleman and Komatsu, in which one looks at the growth of the derivatives on compact sets (see the paper by Komatsu [38] published in 1973), and the theory developed by Björck [3] in 1966 following the ideas previously announced by Beurling in 1961, in which one pays attention to the growth of the Fourier transform of test functions. The point of view of Braun, Meise and Taylor [22] permits a unified treatment of both theories: it is 
strictly broader than Bjork's theory and contains the most relevant cases of Komatsu's theory, in particular, the Gevrey classes. In general, classes defined in one way cannot be defined in another way; see the article of Bonet, Meise and Melikhov [17] for a complete study of the comparison of these two approaches. The theorem of Denjoy-Carleman characterizes non quasianalytic classes versus the quasianalytic case (classes without functions with compact support) in the sense of Carleman and Komatsu.

The idea of Beurling and Björk is the following. Let $\varphi$ be a continuous function with compact support. The function $\varphi$ belongs to $\mathcal{D}\left(\mathbb{R}^{N}\right)$ if and only if the function

$$
\hat{\varphi}(x) \exp \{k \log (1+|x|)\}
$$

is integrable for all $k \in \mathbb{N}_{0}$. In Beurling-Björk's theory the function $\zeta \mapsto$ $\log (1+|\zeta|)$ is replaced by a subadditive weight function $\omega$ which is bigger than $\zeta \mapsto \log (1+|\zeta|)$ in some sense. In this way, the new test space defined by $\omega$ is included in $\mathcal{D}\left(\mathbb{R}^{N}\right)$ and hence its dual (ultradistributions) contains the space of Schwartz distributions. In 1990, Braun, Meise and Taylor replace the subadditivity of the weight by a weaker condition. More precisely, an increasing and continuous function $\omega:[0, \infty[\rightarrow[0, \infty[$ is said to be a weight function if is satisfies the following conditions

$$
\begin{aligned}
& (\alpha) \exists K \geq 1: \omega(2 t) \leq K(1+\omega(t)), \quad(\beta) \quad \int_{1}^{\infty} \frac{\omega(t)}{t^{2}} d t<\infty, \\
& (\gamma) \lim _{t \rightarrow \infty} \frac{\log (1+t)}{\omega(t)}=0, \quad(\delta) \quad \varphi: t \mapsto \omega\left(e^{t}\right) \text { is convex. }
\end{aligned}
$$

Let $\omega$ be a weight function and let $\Omega$ be a non empty open subset of $\mathbb{R}^{N}$. The corresponding test spaces are defined as

$$
\mathcal{D}_{(\omega)}(\Omega):=\left\{f \in \mathcal{D}(\Omega): \int_{\mathbb{R}^{N}}|\hat{f}(t)| e^{k \omega(t)} d t<\infty \text { for all } k>0\right\},
$$

and

$$
\mathcal{D}_{\{\omega\}}(\Omega):=\left\{f \in \mathcal{D}(\Omega): \int_{\mathbb{R}^{N}}|\hat{f}(t)| e^{\varepsilon \omega(t)} d t<\infty \text { for some } \varepsilon>0\right\},
$$

and they are called test spaces of Beurling type and Roumieu type, respectively. Condition $(\beta)$ is called non quasianalytic condition and guarantees that $\mathcal{D}_{(\omega)}(\Omega)$ and $\mathcal{D}_{\{\omega\}}(\Omega)$ are non trivial. Condition $(\delta)$ allows to describe the test spaces by controlling the growth of the derivatives with the Young conjugate as follows,

$$
\mathcal{D}_{(\omega)}(\Omega):=\{f \in \mathcal{D}(\Omega): \forall k \in \mathbb{N},
$$




$$
\left.\sup _{x \in \Omega} \sup _{\alpha \in \mathbb{N}_{0}^{N}}\left|f^{(\alpha)}(x)\right| \exp \left(-k \varphi^{*}\left(\frac{|\alpha|}{k}\right)\right)<\infty\right\}
$$

and

$$
\begin{gathered}
\mathcal{D}_{\{\omega\}}(\Omega):=\{f \in \mathcal{D}(\Omega): \exists k \in \mathbb{N}, \\
\left.\sup _{x \in \Omega} \sup _{\alpha \in \mathbb{N}_{0}^{N}}\left|f^{(\alpha)}(x)\right| \exp \left(-\frac{1}{k} \varphi^{*}(|\alpha| k)\right)<\infty\right\},
\end{gathered}
$$

where $\varphi^{*}$ is the Young conjugate of the convex function $\varphi$. These test spaces induce non quasianalytic classes of ultradifferentiable functions $\mathcal{E}_{(\omega)}(\Omega)$ (Beurling) and $\mathcal{E}_{\{\omega\}}(\Omega)$ (Roumieu) in a similar way as $\mathcal{C}^{\infty}(\Omega)$ is obtained from $\mathcal{D}(\Omega)$.

Given $0<d<1$, the Gevrey class with exponent $s=\frac{1}{d}>1$ is denoted by $\mathcal{G}^{s}(\Omega)$ and it is defined as the space of all smooth functions $f \in \mathcal{C}^{\infty}(\Omega)$ such that for each compact subset $K \subset \subset \Omega$ there is a constant $C>0$ satisfying for each multiindex $\alpha \in \mathbb{N}_{0}^{N}$ the following estimate

$$
\sup _{x \in K}\left|f^{(\alpha)}(x)\right| \leq C^{|\alpha|+1}(\alpha !)^{s} .
$$

Note that for $s=1$, the last estimate characterizes the real analytic functions. If $\omega(t)=t^{d}, 0<d<1$, the space of ultradifferentiable functions of Roumieu type $\mathcal{E}_{\{\omega\}}(\Omega)$ coincides with the classical Gevrey class of exponent $s=\frac{1}{d}>1$. Gevrey classes play an important role in the theory of partial differential operators. It is well known that the heat operator in $\mathbb{R}^{N}, N \geq 2$,

$$
L:=\frac{\partial}{\partial x_{N}}-\sum_{j=1}^{N-1} \frac{\partial^{2}}{\partial x_{j}^{2}},
$$

has a fundamental solution which is smooth in $\mathbb{R}^{N} \backslash\{0\}$ but not real analytic in $x_{N}=0$. However, this fundamental solution belongs to the Gevrey class when $\Omega=\mathbb{R}^{N} \backslash\{0\}$ and $0<d \leq 1 / 2$. Thus, it is natural to consider intermediate classes between $\mathcal{C}^{\infty}(\Omega)$ and the real analytic functions (and their dual spaces) and operators between them.

In this work we consider non quasi analytic classes in the sense of Braun, Meise and Taylor. This point of view and its applications to partial differential operators has received much attention in recent years in the following directions: (1) surjectivity of the operator, i.e., solvability of the underlying equation, (2) existence of a continuous and linear solution operator, (3) study of the range of the operator in case it is not surjective, (4) structure of the kernel of the operator and regularity of the solutions and (5) parameter dependence. We refer [13], [14], [15], [29], [53] and the 
recent paper [11]. The first two volumes of Hörmander [33] constitute a basic reference in these topics.

In the first chapter non quasi analytic classes in the sense of Braun, Meise and Taylor are introduced. We give some notation and background which will be necessary in the sequel.

Classes of $\mathcal{C}^{\infty}$-functions defined in terms of the successive iterates of a partial differential operator appeared in 1960, when Komatsu [37], using tools introduced by Hörmander [32], characterized when a smooth function $f \in \mathcal{C}^{\infty}(\Omega)$ in an open subset $\Omega \subset \mathbb{R}^{N}$ is real analytic in terms of the successive iterates of an elliptic partial differential operator $P(D)$. In particular, given an elliptic differential operator $P(D)$ of order $m$, a function $f \in \mathcal{C}^{\infty}(\Omega)$ is real analytic if and only if for each compact subset $K \subset \subset \Omega$ there exists a constant $C>0$ such that for each $j \in \mathbb{N}_{0}$

$$
\left\|P^{j}(D) f\right\|_{2, K} \leq C^{j+1}(j !)^{m},
$$

where $P^{j}(D)$ is the $j$-th iterate of $P(D)$, i.e., $P^{j}(D)=P(D) \underbrace{\cdots \cdots}_{j} P(D)$ and $\|\cdot\|_{2, K}$ denotes the $\mathcal{L}^{2}$-norm on the compact $K$.

Later, Kotake and Narasimhan gave this result using elliptic operators with analytic coefficients. See [40, Theorem 1].

In 1973, Newberger and Zielezny [57] treated this problem in the setting of the Gevrey classes: let $\mathcal{G}^{s}(\Omega)$ be the Gevrey class of exponent $s>1$ and let $\mathcal{G}_{P}^{s}(\Omega)$ be the class of smooth functions in $\Omega$ such that for each $K \subset \subset \Omega$ there exists a constant $C>0$ such that $\forall j \in \mathbb{N}_{0}$,

$$
\left\|P^{j}(D) f\right\|_{2, K} \leq C^{j+1}(j !)^{s}
$$

then

$$
\mathcal{G}^{s}(\Omega)=\mathcal{G}_{P}^{m s}(\Omega)
$$

whenever $P$ is an elliptic polynomial with degree $m$.

Moreover, in case $P$ and $Q$ are hypoelliptic polynomials, it is proved the equivalence between the inequality $|Q(\xi)|^{2} \leq C\left(1+|P(\xi)|^{2}\right)^{h}, \forall \xi \in$ $\mathbb{R}^{N}$ and the inclusion $\mathcal{G}_{P}^{s}(\Omega) \subset \mathcal{G}_{Q}^{\text {sh }}(\Omega)$ for $s$ large enough.

Classes $\mathcal{G}_{P}^{\text {s }}(\Omega)$ are called generalized Gevrey classes. Métivier [55] proved the converse of the result by Newberger and Zielezny, that is, the equality $\mathcal{G}^{s}(\Omega)=\mathcal{G}_{P}^{m s}(\Omega)$ implies the ellipticity of $P$.

This research is continued by several authors like Bolley, Camus, Rodino $[4,6]$ and Zanghirati $[65,66,67,68]$. We also mention the recent contributions by Bouzar and Chaili [18], Calvo and Hakobyan [23] and Calvo and Rodino [24] where these authors characterize certain Gelfand-Shilov 
spaces in terms of the iterates of a globally elliptic operator $P$ with polynomial coefficients. Langenbruch utilized generalized Gevrey classes in connection with different problems, like boundary values of zero solutions of hypoelliptic differential operators $[45,46]$, diametral dimension of solution spaces [47] and isomorphic classification [48].

Microlocal versions of the problem of iterates have been given by Bolley, Camus and Mattera [5], Zanghirati [68], Bouzar and Chaili [20] in 2006 and others.

The problem of iterates consists in giving conditions on $P$ in order to guarantee the equality $\mathcal{G}^{d}(\Omega)=\mathcal{G}_{P}^{m d}(\Omega)$ or more general in characterizing the functions in a given class of functions in terms of the behavior of the iterates of a fixed differential operator. See [18], [55], [37], [57] and also [19], [24], [40] and [67].

Let $P$ be a linear partial differential operator with constant coefficients. In the second chapter, we introduce classes of smooth functions $\mathcal{E}_{P,(\omega)}(\Omega)$ and $\mathcal{E}_{P,\{\omega\}}(\Omega)$ of Beurling and Romieu type involving the successive iterates of the operator $P$, that is, satisfying for each $j \in \mathbb{N}_{0}$ the following type estimates

$$
\left\|P^{j}(D) f\right\|_{2, K} \leq C \exp \left(-\lambda \varphi^{*}\left(\frac{j}{\lambda}\right)\right)
$$

where $K$ is a compact subset in $\Omega$ and $\lambda$ a positive number. We call $\mathcal{E}_{P,(\omega)}(\Omega)$ and $\mathcal{E}_{P,\{\omega\}}(\Omega)$ generalized non quasianalytic classes. The completeness of these spaces is characterized in terms of the hypoellipticity of $P$. For this class of polynomials the nuclearity of $\mathcal{E}_{P,(\omega)}(\Omega)$ and $\mathcal{E}_{P,\{\omega\}}(\Omega)$ is proved. After that, we establish a Paley-Wiener type theorem for $\mathcal{E}_{P,(\omega)}(\Omega)$ and $\mathcal{E}_{P,\{\omega\}}(\Omega)$. As a consequence, we show that the test functions of $\mathcal{E}_{P,(\omega)}(\Omega)$ and $\mathcal{E}_{P,\{\omega\}}(\Omega)$ are nuclear spaces and give a sufficient condition to ensure that these test functions are an algebra.

Chapter 3 is devoted to extend results of Komatsu [37] and NewbergerZielezny [57] to the ultradifferentiable setting. Moreover, we treat the problem of iterates on non quasianalytic classes. In fact, for weights $\omega$ satisfying a certain growth condition, we show that the non quasianalytic class of ultradifferentiable functions $\mathcal{E}_{(\omega)}(\Omega)$ (or $\mathcal{E}_{\{\omega\}}(\Omega)$ ) coincides with a generalized non quasianalytic class defined by the polinomial $P$ if and only if $P$ is elliptic, thus extending a result by Métivier [55]. If the weight function is a power of the logarithm, we show that the equality between the corresponding class of ultradifferentiable functions and the generalized non quasianalytic class defined by $P$ is characterized by the hypoellipticity of $P$, thus showing that the growth condition above is needed to get the ellipticity of $P$. 
In 2000, Langenbruch and Voigt proved the following result: a Fréchet space of distributions which is stable under differential operators is continuously included in $\mathcal{C}^{\infty}$. These authors proved that in order to guarantee this continuous inclusion it is enough to assume that the Fréchet space is stable under a single hypoelliptic differential operator. In Chapter 4 we give extensions of this result to the ultradifferentiable setting using strongly $(\omega)$-hypoelliptic operators. We also show the connections of this topic with the problem of iterates of differential operators. For instance, we introduce the notion of Fréchet space $(\omega, P(D))$-stable in order to prove that a Fréchet space of ultradistributions invariant under some class of hypoelliptic, semi-elliptic or elliptic differential operators is formed by ultradifferentiable functions. The notion of $(\omega, P(D))$-stability involves an equicontinuity condition on the successive iterates of $P(D)$.

Vector valued distributions and vector valued ultradifferentiable functions also have received much attention in recent papers. This research began in 1957 with the work by Schwartz [60, 61]. Komatsu studied in 1982 [39] classes of ultradifferentiable functions or ultradistributions with values in a sequentially complete topological vector space, thus extending the previous work of Schwartz.

In 1990, Kriegl and Michor introduced several notions of real analytic curves in a locally convex space $E$, i.e, vector valued maps $f: \mathbb{R} \rightarrow E$ which are real analytic (see [42]). This research was motivated by applications to differential and analytic manifolds; see [43]. Real analytic curves with values in Fréchet or (LB)-spaces were studied by Bonet and Domanski in several papers since 1998 [9, 10,12]. In particular, these authors clarified the relation between various notions of real analyticity introduced by Kriegl and Michor [9]. Their main tool is the modern theory of Fréchet and LB-spaces. Moreover, Bonet and Domanski have applied their results to investigate surjective linear partial differential operators between spaces of vector valued real analytic functions and parameter dependence of the solutions of these operators $[9,10,11,12]$.

Kriegl, Michor and Rainer [44] have studied recently non-quasianalytic curves $f: \mathbb{R} \rightarrow E$ with values in a locally convex space $E$ in the setting of non-quasianalytic classes of Denjoy-Carleman type.

Let $\omega$ be a weight function in the sense of Brau, Meise and Taylor. In the last chapter, vector valued $\omega$-ultradifferentiable functions $f: \Omega \rightarrow E$ with values in a locally convex space $E$ are considered. As in the cases of real analytic or non-quasianalytic curves treated by Kriegl, Michor and Rainer, three possible notions are given. Solving a problem posed by Kriegl and Michor in a meeting celebrated in Paderborn (Germany) in November 2008, it is proved that every weakly non-quasianalytic ultra- 
differentiable function with values in a Fréchet space $E$ is topologically (or strongly) ultradifferentiable if and only if the space $E$ satisfies the topological invariant $(\mathrm{DN})$ of Vogt. We also treat the case that $E$ is a complete $L B$-space. 



\section{Chapter 1}

\section{Preliminaries: non-quasianalytic classes}

In this chapter, non quasianalytic classes in the sense of Braun, Meise and Taylor are introduced. We establish the definitions and the fundamental properties that we shall need through this thesis. We follow [22].

Definition 1.0.1. A non-quasi-analytic weight function is an increasing continuous function $\omega:[0, \infty[\rightarrow[0, \infty$ [ with the following properties:

$(\alpha)$ there exists $L \geq 0$ with $\omega(2 t) \leq L(\omega(t)+1)$ for all $t \geq 0$,

( $\beta) \int_{1}^{\infty} \frac{\omega(t)}{t^{2}} d t<\infty$,

$(\gamma) \log (t)=o(\omega(t))$ as $t$ tends to $\infty$, that is, $\lim _{t \rightarrow \infty} \frac{\log (t)}{\omega(t)}=0$.

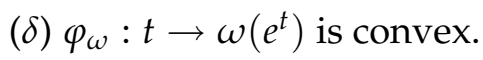

Normally, we will denote $\varphi_{\omega}$ simply by $\varphi$.

For a weight function $\omega$ we define $\bar{\omega}: \mathbb{C}^{N} \rightarrow[0,+\infty[$ by $\bar{\omega}(z):=\omega(|z|)$ and again we denote this function by $\omega$.

The condition $(\beta)$ is called non-quasianalytic condition and it implies $\omega(t)=o(t)$. Moreover, this condition implies the existence of functions with compact support in the class of ultradifferentiable functions that we will define later. 
Definition 1.0.2. The Young conjugate $\varphi^{*}:[0, \infty[\rightarrow \mathbb{R}$ of an increasing and convex function $\varphi$ with $\varphi(0)=0$ and $\lim _{x \rightarrow \infty} \frac{x}{\varphi(x)}=0$ is given by

$$
\varphi^{*}(s):=\sup \{s t-\varphi(t), t \geq 0\} .
$$

Given a weight function $\omega$, there exists another weight function $\sigma$ such that $\sigma(t)=\omega(t)$ for $t>0$ large enough and $\sigma(t)=0$ if $t \in[0,1]$. Therefore, there is no loss of generality to assume that $\omega$ vanishes on $[0,1]$. Then $\varphi^{*}$ has only non-negative values, it is convex and $\varphi^{*}(t) / t$ is increasing and tends to $\infty$ as $t \rightarrow \infty$ and $\varphi^{* *}=\varphi$.

Lemma 1.0.3. Given $\varphi$ as above, by condition $(\alpha)$ of weight function, there exists $L \geq 0$ such that

$$
\varphi(x+1) \leq L(1+\varphi(x))
$$

for all $x \in\left[0, \infty\left[\right.\right.$. Then, there exists $y_{0}>0$ such that

$$
\varphi^{*}(y)-y \geq L \varphi^{*}\left(\frac{y}{L}\right)-L
$$

for all $y \geq y_{0}$.

Lemma 1.0.4. Given $\lambda>0$ there exists a constant $C>0$ (depending on $\lambda)$ such that

$$
\exp \left(2 \lambda \varphi^{*}\left(\frac{x+1}{2 \lambda}\right)\right) \leq C \exp \left(\lambda \varphi^{*}\left(\frac{x}{\lambda}\right)\right) \forall x>0 .
$$

Proof. From the convexity of $\varphi^{*}$ we obtain

$$
\varphi^{*}\left(\frac{x}{2 \lambda}+\frac{1}{2 \lambda}\right) \leq \frac{1}{2} \varphi^{*}\left(\frac{x}{\lambda}\right)+\frac{1}{2} \varphi^{*}\left(\frac{1}{\lambda}\right) .
$$

The conclusion follows with $C=\exp \left(\lambda \varphi^{*}\left(\frac{1}{\lambda}\right)\right)$.

Example 1.0.5. The following functions are, after a change in some interval $[0, M]$, examples of weight functions:

(i) $\omega(t)=t^{d}$ for $0<d<1$.

(ii) $\omega(t)=(\log (1+t))^{s}, s>1$.

(iii) $\omega(t)=t(\log (e+t))^{-\beta}, \beta>1$.

(iv) $\omega(t)=\exp \left(\beta(\log (1+t))^{\alpha}\right), 0<\alpha<1$.

Let $\mathbb{N}_{0}=\mathbb{N} \cup\{0\}$ be the set of non negative integers. Then, let us denote by $\mathbb{N}_{0}^{N}$ the set of $N$-tuples. For each $\alpha=\left(\alpha_{1}, \ldots, \alpha_{N}\right) \in \mathbb{N}_{0}^{N}$ we write 


$$
f^{(\alpha)}=\left(\partial / \partial x_{1}\right)^{\alpha_{1}} \cdots\left(\partial / \partial x_{N}\right)^{\alpha_{N}} f
$$

and using the notation $D_{x_{j}}=-i \partial / \partial x_{j}$, where $i$ is the imaginary unit, we write

$$
D^{\alpha} f=D_{x_{1}}^{\alpha_{1}} \cdots D_{x_{N}}^{\alpha_{N}} f .
$$

In what follows, $\Omega$ denotes an arbitrary subset of $\mathbb{R}^{N}$ and $K \subset \subset \Omega$ means that $K$ is a compact subset in $\Omega$.

Definition 1.0.6. Let $\omega$ be a weight function.

(a) For a compact subset $K$ in $\mathbb{R}^{N}$ which coincides with the closure of its interior and $\lambda>0$, define the seminorm

$$
p_{K, \lambda}(f):=\sup _{x \in K} \sup _{\alpha \in \mathbb{N}_{0}^{N}}\left|f^{(\alpha)}(x)\right| \exp \left(-\lambda \varphi^{*}\left(\frac{|\alpha|}{\lambda}\right)\right),
$$

and set

$$
\mathcal{E}_{\omega}^{\lambda}(K):=\left\{f \in C^{\infty}(K): p_{K, \lambda}(f)<\infty\right\},
$$

which is a Banach space endowed with the $p_{K, \lambda}(\cdot)$-topology.

(b) For an open subset $\Omega$ in $\mathbb{R}^{N}$, the class of $\omega$-ultradifferentiable functions of Beurling type is defined by:

$\mathcal{E}_{(\omega)}(\Omega):=\left\{f \in C^{\infty}(\Omega): p_{K, \lambda}(f)<\infty\right.$, for every $K \subset \subset \Omega$ and every $\left.\lambda>0\right\}$.

The topology of this space is

$$
\mathcal{E}_{(\omega)}(\Omega)=\underset{K \subset \subset \Omega}{\operatorname{proj}} \underset{\lambda>0}{\operatorname{proj}} \mathcal{E}_{\omega}^{\lambda}(K)
$$

This topology is also described by the fundamental system of seminorms given by $\left\{p_{K_{n}, n}\right\}_{n \in \mathbb{N}}$ where $\left\{K_{n}\right\}_{n \in \mathbb{N}}$ is a compact exhaustion of $\Omega$. One can show that $\mathcal{E}_{(\omega)}(\Omega)$ is a Fréchet space, that is, a complete and metrizable locally convex space. Moreover, $\mathcal{E}_{(\omega)}(\Omega)$ is reflexive and nuclear. We denote by $\mathcal{E}_{(\omega)}(\Omega)_{\beta}^{\prime}$ the strong dual of $\mathcal{E}_{(\omega)}(\Omega)$.

(c) For a compact subset $K$ in $\mathbb{R}^{N}$ which coincides with the closure of its interior and $\lambda>0$, set

$$
\mathcal{E}_{\{\omega\}}(K)=\left\{f \in C^{\infty}(K): \text { there exists } m \in \mathbb{N} \text { such that } p_{K, \frac{1}{m}}(f)<\infty\right\}
$$


This space is the strong dual of a nuclear Fréchet space (i.e, a (DFN)-space) if it is endowed with its natural inductive limit topology, that is,

$$
\mathcal{E}_{\{\omega\}}(K)=\underset{m \in \mathbb{N}}{\underset{m}{\longrightarrow}} \mathcal{E}_{\omega}^{\frac{1}{m}}(K) .
$$

(d) For an open subset $\Omega$ in $\mathbb{R}^{N}$, the class of $\omega$-ultradifferentiable functions of Roumieu type is defined by:

$$
\mathcal{E}_{\{\omega\}}(\Omega):=\left\{f \in C^{\infty}(\Omega): \forall K \subset \subset \Omega \exists \lambda>0 \text { such that } p_{K, \lambda}(f)<\infty\right\} .
$$

Its topology is the following

$$
\mathcal{E}_{\{\omega\}}(\Omega)=\underset{K \subset \subset \Omega}{\operatorname{proj}} \mathcal{E}_{\{\omega\}}(K)
$$

that is, it is endowed with the topology of the projective limit of the spaces $\mathcal{E}_{\{\omega\}}(K)$ when $K$ runs the compact subsets of $\Omega$. This is a complete PLSspace, that is, a complete space which is a projective limit of LB-spaces (i.e., a countable inductive limit of Banach spaces) with compact linking maps in the (LB)-steps. Moreover, $\mathcal{E}_{\{\omega\}}(\Omega)$ is also a nuclear and reflexive locally convex space. In particular, $\mathcal{E}_{\{\omega\}}(\Omega)$ is an ultrabornological (hence barrelled and bornological) space. We denote by $\mathcal{E}_{\{\omega\}}(\Omega)_{\beta}^{\prime}$ the strong dual of $\mathcal{E}_{\{\omega\}}(\Omega)$.

The elements of $\mathcal{E}_{(\omega)}(\Omega)$ (resp. $\mathcal{E}_{\{\omega\}}(\Omega)$ ) are called ultradifferentiable functions of Beurling type (resp. Roumieu type) in $\Omega$.

In the case that $\omega(t):=t^{d}(0<d<1)$, the corresponding Roumieu class is the Gevrey class with exponent $1 / d$. In the limit case $d=1$, not included in our setting, the corresponding Roumieu class $\mathcal{E}_{\{\omega\}}(\Omega)$ is the space of real analytic functions on $\Omega$ whereas the Beurling class $\mathcal{E}_{(\omega)}\left(\mathbb{R}^{N}\right)$ gives the entire functions.

If a statement holds in the Beurling and the Roumieu case then we will use the notation $\mathcal{E}_{*}(\Omega)$. It means that in all cases * can be replaced either by $(\omega)$ or $\{\omega\}$.

Definition 1.0.7. Two weight functions $\omega$ and $\sigma$ are said to be equivalent if $\omega=O(\sigma)$ and $\sigma=O(\omega)$. 
Remark 1.0.8. Let us consider $\omega$ and $\sigma$ two equivalent weight functions and let us denote $\varphi(x)=\omega\left(e^{x}\right)$ and $\psi(x)=\sigma\left(e^{x}\right)$. If $\omega \leq \sigma \leq C \omega$ for some constant $C>0$, then

$$
C \varphi^{*}(y / C) \leq \psi^{*}(y) \leq \varphi^{*}(y), \quad y \geq 0 .
$$

As a consequence $\omega$ and $\sigma$ define the same class of ultradifferentiable functions.

The corresponding classes of test functions are defined as follows:

Definition 1.0.9. For a compact set $K$ in $\mathbb{R}^{N}$, define

$$
\mathcal{D}_{*}(K):=\left\{f \in \mathcal{E}_{*}\left(\mathbb{R}^{N}\right): \operatorname{supp} f \subset K\right\},
$$

endowed with the induced topology. Recall that the condition $(\beta)$ guarantees that $\mathcal{D}_{*}(K) \neq\{0\}$. In fact, in [22, Remark 3.2 (1) and Corollary 3.6 (1)] it is shown that $\mathcal{D}_{*}(K) \neq\{0\}$. $\mathcal{D}_{(\omega)}(K)$ is a Fréchet space while $\mathcal{D}_{\{\omega\}}(K)$ is the strong dual of a nuclear Fréchet space (i.e., it is a (DFN)-space). For an open set $\Omega$ in $\mathbb{R}^{N}$, define

$$
\mathcal{D}_{*}(\Omega):=\underset{K \subset \subset \Omega}{\underset{\text { ind }}{\longrightarrow}} \mathcal{D}_{*}(K) .
$$

According to [22, Proposition 4.7], the following inclusion

$$
\mathcal{D}_{*}(\Omega) \hookrightarrow \mathcal{E}_{*}(\Omega)
$$

is continuous with dense range.

Next lemma is well-known, but it is not easy to find a precise reference.

Lemma 1.0.10. The spaces $\mathcal{E}_{*}(\Omega)$ and $\mathcal{D}_{*}(\Omega)$ can be described with $\mathcal{L}^{2}$-norms, i.e., we can replace $p_{K, \lambda}$ by the seminorms

$$
q_{K, \lambda}(f):=\sup _{\alpha \in \mathbb{N}_{0}^{N}}\left\|f^{(\alpha)}\right\|_{2, K} \exp \left(-\lambda \varphi^{*}\left(\frac{|\alpha|}{\lambda}\right)\right)
$$

where

$$
\|f\|_{2, K}=\left(\int_{K}|f|^{2}\right)^{\frac{1}{2}} .
$$

Proof. We only need to prove that for each compact subset $K \subset \subset \Omega$ and $\lambda>0$, there is other compact subset $L \subset \subset \Omega, \mu>0$ and a constant $D>0$ (depending only on $K$ and $\lambda$ ) such that for all $f \in \mathcal{E}_{*}(\Omega)$,

$$
p_{K, \lambda}(f) \leq D q_{L, \mu}(f)
$$


We fix $K \subset \subset \Omega$ and $\lambda>0$. We take $L \subset \subset \Omega$ such that $K \subset \stackrel{\circ}{L} \subset \Omega$, where $\stackrel{\circ}{L}$ denotes the interior set of $L$. By the Sobolev Lemma, there exists a constant $C>0$ such that

$$
\sup _{x \in K}|f(x)| \leq C \sup _{|\beta| \leq N+1} \int_{L}\left|f^{(\beta)}\right| \forall f \in \mathcal{C}^{\infty}(\Omega) .
$$

Then,

$$
\begin{gathered}
\sup _{x \in K}\left|f^{(\alpha)}(x)\right| \leq C \sup _{|\beta| \leq N+1} \int_{L}\left|f^{(\alpha+\beta)}\right|= \\
=C \sup _{|\beta| \leq N+1} \int_{L}\left|f^{(\alpha+\beta)}\right| \exp \left(-\lambda \varphi^{*}\left(\frac{|\alpha|+|\beta|}{\lambda}\right)\right) \exp \left(\lambda \varphi^{*}\left(\frac{|\alpha|+|\beta|}{\lambda}\right)\right) \leq \\
\leq C_{2} q_{L, \lambda}(f) \exp \left(\lambda \varphi^{*}\left(\frac{|\alpha|+N+1}{\lambda}\right)\right) \leq C_{3} q_{L, \lambda}(f) \exp \left(\frac{\lambda}{2^{N+1}} \varphi^{*}\left(\frac{2^{N+1}}{\lambda}|\alpha|\right)\right) .
\end{gathered}
$$

where we have applied that $\varphi^{*}$ is increasing, Lemma 1.0.4 and Hölder's inequality.

As a consequence,

$$
p_{K, \frac{\lambda}{2^{N+1}}}(f) \leq C_{3} q_{L, \lambda}(f)
$$

So, given $\lambda>0$ we take $\mu=2^{N+1} \lambda$.

The elements of the topological dual $\mathcal{D}_{(\omega)}(\Omega)^{\prime}$ (resp. $\left.\mathcal{D}_{\{\omega\}}(\Omega)^{\prime}\right)$ are called ultradistributions of Beurling type (resp. Roumieu type). Since $\mathcal{D}_{(\omega)}(\Omega) \subset \mathcal{D}_{\{\omega\}}(\Omega)$ is a continuous and dense inclusion, $\mathcal{D}_{\{\omega\}}(\Omega)^{\prime}$ is a subspace of $\mathcal{D}_{(\omega)}(\Omega)^{\prime}$.

Let $T \in \mathcal{D}_{*}(\Omega)^{\prime}$. The support of $T$ is defined as

$$
\begin{array}{ll}
\operatorname{supp}_{*} T:=\{x \in \Omega \quad & \text { for each neighborhood } U \text { of } x \\
& \text { there is } \left.\varphi \in \mathcal{D}_{*}(U) \text { such that }<T, \varphi>\neq 0\right\} .
\end{array}
$$

If $T \in \mathcal{D}_{\{\omega\}}^{\prime}(\Omega)$ then $\operatorname{supp}_{(\omega)} T=\operatorname{supp}_{\{\omega\}} T$.

Moreover, if $\omega=o(\sigma)$, then $\mathcal{D}_{\{\sigma\}}(\Omega) \subset \mathcal{D}_{(\omega)}(\Omega)$ with continuous and dense inclusion ([22, Proposition 3.9]). As a consequence, $\mathcal{D}_{(\omega)}(\Omega)^{\prime} \subset$ $\mathcal{D}_{\{\sigma\}}(\Omega)^{\prime}$. If $T \in \mathcal{D}_{\{\omega\}}^{\prime}(\Omega)$,

$$
\operatorname{supp}_{(\omega)} T=\operatorname{supp}_{\{\omega\}} T=\operatorname{supp}_{\{\sigma\}} T
$$

is fulfilled. 
An ultradistribution $\mu \in \mathcal{D}_{*}(\Omega)^{\prime}$ can be continuously extended to $\mathcal{E}_{*}(\Omega)^{\prime}$ if, and only if, $\mu$ has compact support in $\Omega$. The space of ultradistributions, having compact support, of Beurling type (resp. Roumieu type) is denoted by $\mathcal{E}_{(\omega)}(\Omega)^{\prime}$ (resp. by $\left.\mathcal{E}_{\{\omega\}}(\Omega)^{\prime}\right)$.

Now, we introduce the convolution of ultradistributions:

Definition 1.0.11. Let $\mu \in \mathcal{E}_{*}\left(\mathbb{R}^{N}\right)^{\prime}$ be given and suppose $\mu \neq 0$. Define:

$$
S_{\mu}: \mathcal{D}_{*}\left(\mathbb{R}^{N}\right)^{\prime} \longrightarrow \mathcal{D}_{*}\left(\mathbb{R}^{N}\right)^{\prime}, S_{\mu}(E):=\mu * E,
$$

where $\left\langle\mu * E, \varphi>=<E, \check{\mu} * \varphi>\right.$ and $\check{\mu} * \varphi: x \rightarrow \mu(\varphi(x+\cdot)), x \in \mathbb{R}^{N}$. Then, $S_{\mu}$ is a continuous and linear operator.

If $E \in \mathcal{E}_{*}\left(\mathbb{R}^{N}\right)$, then $\mu * E \in \mathcal{E}_{*}\left(\mathbb{R}^{N}\right)$. Let us denote by $T_{\mu}: \mathcal{E}_{*}\left(\mathbb{R}^{N}\right) \longrightarrow$ $\mathcal{E}_{*}\left(\mathbb{R}^{N}\right)$ the restriction of $S_{\mu}$ to $\mathcal{E}_{*}\left(\mathbb{R}^{N}\right)$.

The following proposition is used in the next chapter. See [22, Proposition 6.4].

Proposition 1.0.12. $\mathcal{D}_{*}\left(\mathbb{R}^{N}\right) * \mathcal{D}_{*}\left(\mathbb{R}^{N}\right)^{\prime}$ is a subset of $\mathcal{E}_{*}\left(\mathbb{R}^{N}\right)$. For $f \in \mathcal{D}_{*}\left(\mathbb{R}^{N}\right)$ and $E \in \mathcal{D}_{*}\left(\mathbb{R}^{N}\right)^{\prime}$ we have $f * E(x)=\left\langle E_{y}, f(x-y)\right\rangle$.

Definition 1.0.13. Let $f \in \mathcal{D}_{*}(\Omega)^{\prime}$. The singular support of $f$ with respect to $*$, denoted by $\sin _{*} \operatorname{supp} f$, is the complementary set of the biggest open set $A$ in $\Omega$ where $f \in \mathcal{E}_{*}(A)$.

Now, we recall Paley-Wiener type theorems in the ultradifferentiable setting: the Fourier-Laplace transform establishes an isomorphism between $\mathcal{D}_{(\omega)}(\Omega)$ and certain weighted spaces of holomorphic functions. $\mathcal{E}_{(\omega)}\left(\mathbb{R}^{N}\right)^{\prime}$ also may be interpreted as a space of holomorphic functions.

Definition 1.0.14. The Fourier-Laplace transform of a function $\varphi \in \mathcal{D}(\Omega)$ is defined as $\hat{\varphi}: \mathbb{C}^{N} \rightarrow \mathbb{C}$, where

$$
\hat{\varphi}(z):=\int_{\mathbb{R}^{N}} e^{-i x \cdot z} \varphi(x) d x, \quad z \in \mathbb{C}^{N},
$$

and $x \cdot z=x_{1} \cdot z_{1}+\cdots+x_{N} \cdot z_{N}$.

The Fourier-Laplace transform of $\varphi$ is an entire function. In fact, it is the unique entire function on $\mathbb{C}^{N}$ whose restriction to $\mathbb{R}^{N}$ coincides with the Fourier transform of $\varphi$.

Let $K \subset \mathbb{R}^{N}$ be a convex compact subset. Then,

$$
H_{K}(x):=\sup _{y \in K} x \cdot y, \quad x \in \mathbb{R}^{N},
$$


Preliminaries: non-quasianalytic classes

is called supporting function of $K$.

A Paley-Wiener type theorem in the classic case is proved in [33, I,Theorem 7.3.1]. With respect to test functions on non quasi analytic classes, the following theorem holds.

Theorem 1.0.15. Let $K \subset \mathbb{R}^{N}$ be a convex compact subset. An entire function $h \in \mathcal{H}\left(\mathbb{C}^{N}\right)$ is the Fourier-Laplace transform of $\varphi \in \mathcal{D}_{(\omega)}(\Omega)$ with supp $\varphi \subset K$ if and only if for any $n \in \mathbb{N}$ there exists a positive constant $C_{n}>0$ such that

$$
|h(z)| \leq C_{n} \exp \left(H_{K}(|\operatorname{Imz}|)-n \omega(z)\right), \quad z \in \mathbb{C}^{N} .
$$

As a consequence, the topology of the space $\mathcal{D}_{(\omega)}(K)$ introduced in the definition 1.0.9 is given by the fundamental system of seminorms

$$
\|f\|_{m}:=\int|\hat{f}(\xi)| e^{m \omega(\xi)} d \xi, \quad m \in \mathbb{N} .
$$

Definition 1.0.16. (1) For each $z \in \mathbb{C}^{N}$ let us denote

$$
v_{z}(x)=\exp (-i x \cdot z), \quad x \in \mathbb{R}^{N} .
$$

(2) We define the Fourier-Laplace transform $\hat{\mu}$ of $\mu \in \mathcal{E}_{(\omega)}\left(\mathbb{R}^{N}\right)^{\prime}$ as

$$
\hat{\mu}(z)=<\mu, v_{z}>\text {. }
$$

Theorem 1.0.17. [Paley-Wiener-Schwartz] Let $K \subset \mathbb{R}^{N}$ be a convex compact subset. For any entire function $f$ and any weight function $\omega$, the following statements are equivalent:

(1) There exists an ultradistribution $\mu \in \mathcal{E}_{(\omega)}\left(\mathbb{R}^{N}\right)^{\prime}$ with supp $\mu \subset K$ such that $\hat{\mu}=f$.

(2) There exists $\lambda>0$ such that for all $\varepsilon>0$ there exists $C>0$ in such a way

$$
|f(z)| \leq C \exp \left(H_{K}(\operatorname{Im} z)+\varepsilon|\operatorname{Im} z|+\lambda \omega(\operatorname{Rez})\right), \quad z \in \mathbb{C}^{N},
$$

is fulfilled.

(3) There exists $\lambda>0$ such that for all $\varepsilon>0$ there exists $C>0$ in such a way

$$
\int_{\mathbb{R}^{N}}|f(x+i y)| e^{-\lambda \omega(x)} d x \leq C e^{H_{K}(y)+\varepsilon|y|}, \quad y \in \mathbb{R}^{N},
$$

is fulfilled.

In this thesis, we consider non quasi analytic classes of ultradifferentiable functions in the sense of Braun, Meise and Taylor but it is convenient to have in mind classes of ultradifferentiable functions in the sense of Carleman and Komatsu [38]. These classes are defined by weight sequences. 
Definition 1.0.18. A sequence of positive numbers $\left(M_{p}\right)_{p \in \mathbb{N}_{0}}$ is called weight sequence if it satisfies the following conditions:

$\left(M_{0}\right)$ There exists $c>0$ such that $(c(p+1))^{p} \leq M_{p}, \quad p \in \mathbb{N}_{0}$.

$\left(M_{1}\right) M_{p}^{2} \leq M_{p-1} M_{p+1}$ for all $p \in \mathbb{N}_{0}$ and $M_{0}=1$.

$\left(M_{2}\right)$ / There are $A, H>1$ such that $M_{p+1} \leq A H^{p} M_{p}, \quad p \in \mathbb{N}_{0}$.

A weight sequence is called non quasianalytic if it satisfies

$$
\sum_{p=1}^{\infty} \frac{M_{p-1}}{M_{p}}<\infty
$$

otherwise it is called quasianalytic.

Definition 1.0.19. Let $\left(M_{p}\right)_{p \in \mathbb{N}_{0}}$ be a weight sequence and let $\Omega$ be an open subset in $\mathbb{R}^{N}$. For each compact subset $K$ in $\Omega$ and each positive number $h>0$, consider the seminorm

$$
p_{K, h}(f)=\sup _{x \in K} \sup _{\alpha \in \mathbb{N}_{0}^{N}} \frac{\left|f^{(\alpha)}(x)\right|}{h^{|\alpha|} M_{\alpha}} .
$$

The Carlemann class $\mathcal{E}_{\left(M_{p}\right)}(\Omega)$ of Beurling type is defined as

$$
\begin{aligned}
\mathcal{E}_{\left(M_{p}\right)}(\Omega):= & \left\{f \in C^{\infty}(\Omega): \text { for every } K \subset \subset \Omega\right. \text { and each } \\
& \left.h>0, p_{K, h}(f)<\infty\right\},
\end{aligned}
$$

and the Carlemann class of Roumieu type

$$
\begin{aligned}
\mathcal{E}_{\left\{M_{p}\right\}}(\Omega):= & \left\{f \in C^{\infty}(\Omega): \text { for every } K \subset \subset \Omega\right. \text { there exists } \\
& \left.h>0 \text { such that } p_{K, h}(f)<\infty\right\} .
\end{aligned}
$$

Bonet, Meise and Melikhov characterize in [17] the weight sequences such that the corresponding class of ultradifferentiable functions coincide with a class of ultradifferentiable functions in the sense of Braun, Meise and Taylor. They also characterize those weight functions $\omega$ for which there exists a weight sequence $\left(M_{p}\right)_{p \in \mathbb{N}_{0}}$ such that the classes defined by $\omega$ and by $\left(M_{p}\right)_{p \in \mathbb{N}_{0}}$, respectively, coincide. 



\section{Chapter 2}

\section{Generalized non-quasianalytic classes}

Let $P$ be a linear partial differential operator with constant coefficients. In this chapter, we introduce classes of smooth functions $\mathcal{E}_{P,(\omega)}(\Omega)$ and $\mathcal{E}_{P,\{\omega\}}(\Omega)$ of Beurling and Romieu type involving the successive iterates of the operator $P$. This classes have nice topological properties when the polynomial $P$ is hypoelliptic. In Theorem 2.2 .5 we prove that $\mathcal{E}_{P,(\omega)}(\Omega)$ and $\mathcal{E}_{P,\{\omega\}}(\Omega)$ are complete if and only if $P$ is hypoelliptic. Moreover, if $P$ is hypoelliptic we prove that $\mathcal{E}_{P,(\omega)}(\Omega)$ and $\mathcal{E}_{P,\{\omega\}}(\Omega)$ are nuclear and a Paley-Wiener type theorem for these generalized non quasi analytic classes is established. These are the contents of Theorem 2.1.11 and Theorem 2.3.10.

\subsection{The class $\mathcal{E}_{P, \omega}(\Omega)$}

We consider smooth functions in an open set $\Omega$ such that $\exists C>0$ verifying for each $j \in \mathbb{N}_{0}$

$$
\left\|P^{j}(D) f\right\|_{2, K} \leq C \exp \left(\lambda \varphi^{*}\left(\frac{j}{\lambda}\right)\right),
$$

where $K$ is a compact subset in $\Omega$ and $P^{j}(D)$ is the $j$-th iterate of the partial differential operator $P(D)$, i.e,

$$
P^{j}(D)=P(D) \underbrace{\rho \cdots \circ}_{j} P(D) .
$$

If $j=0$, then

$$
P^{0}(D) f=f .
$$


Given a polynomial $P \in \mathbb{C}\left[z_{1}, \ldots, z_{N}\right]$ with degree $m$,

$$
P(z)=\sum_{|\alpha| \leq m} a_{\alpha} z^{\alpha}
$$

the partial differential operator $P(D)$ is the following:

$$
P(D)=\sum_{|\alpha| \leq m} a_{\alpha} D^{\alpha}, \text { where } D^{\alpha}=\frac{1}{i} \partial^{\alpha} .
$$

The spaces of ultradifferentiable functions with respect to the successive iterates of $P$ are defined as follows:

Let $\omega$ be a weight function. Given a polynomial $P$, an open set $\Omega$ of $\mathbb{R}^{N}$, a compact subset $K \subset \subset \Omega$ and $\lambda>0$, we define the seminorm

$$
\|f\|_{K, \lambda}:=\sup _{j \in \mathbb{N}_{0}}\left\|P^{j}(D) f\right\|_{2, K} \exp \left(-\lambda \varphi^{*}\left(\frac{j}{\lambda}\right)\right)
$$

and set

$$
\mathcal{E}_{P, \omega}^{\lambda}(K)=\left\{f \in \mathcal{C}^{\infty}(K):\|f\|_{K, \lambda}<+\infty\right\} .
$$

$\mathcal{E}_{P, \omega}^{\lambda}(K)$ is a Banach space endowed with the $\|\cdot\|_{K, \lambda}$-norm.

The space of ultradifferentiable functions of $P$ of Beurling type with respect to the iterates is:

$$
\mathcal{E}_{P,(\omega)}(\Omega)=\left\{f \in \mathcal{C}^{\infty}(\Omega):\|f\|_{K, \lambda}<+\infty \text { for each } K \subset \subset \Omega \text { and } \lambda>0\right\} .
$$

It is endowed with the topology given by

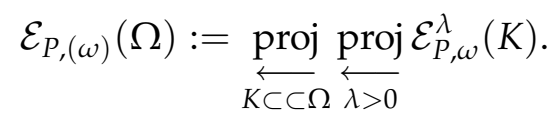

If $\left\{K_{n}\right\}_{n \in \mathbb{N}}$ is a compact exhaustion of $\Omega$ we have

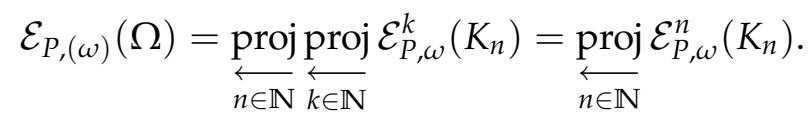

This metrizable locally convex topology is defined by the fundamental system of seminorms $\left\{\|\cdot\|_{K_{n}, n}\right\}_{n \in \mathbb{N}}$.

The space of ultradifferentiable functions of Roumieu type with respect to the iterates of $P$ is defined by:

$$
\mathcal{E}_{P,\{\omega\}}(\Omega)=\left\{f \in \mathcal{C}^{\infty}(\Omega): \forall K \subset \subset \Omega \exists \lambda>0 \text { such that }\|f\|_{K, \lambda}<+\infty\right\} .
$$


Its topology is defined by

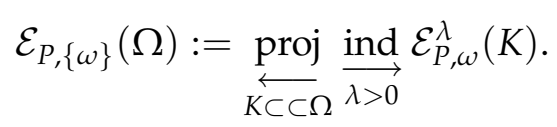

As in the Gevrey case, we call theses classes generalized non quasi analytic classes. Inspired by previous papers we have defined the generalized non quasi analytic classes in terms of $\mathcal{L}^{2}$-norms. Now we show in case that the polynomial $P$ is hypoelliptic, we can replace the $\mathcal{L}^{2}$-norms by $\mathcal{L}^{p}$. norms where $1 \leq p \leq \infty$. Previously, we need some preliminaries on Hörmander's $\mathcal{B}_{p, k}$ spaces. We follow the chapters $\mathrm{X}$ and XI of [33, II].

Definition 2.1.1. A positive function $k$ defined in $\mathbb{R}^{N}$ is said to be a temperate weight function if there exist positive constants $C>0$ and $c>0$ such that

$$
k(\xi+\mu) \leq(1+C|\xi|)^{c} k(\mu), \quad \xi, \mu \in \mathbb{R}^{N} .
$$

Let $P$ be a polynomial. Let us denote $P^{(\alpha)}=\partial^{(\alpha)} P$. The basic example of temperate weight function is the function $\widetilde{P}$ defined as

$$
\widetilde{P}(\xi)=\left(\sum_{\alpha}\left|P^{(\alpha)}(\xi)\right|^{2}\right)^{\frac{1}{2}}, \xi \in \mathbb{R}^{N} .
$$

Note that the sum is finite.

Definition 2.1.2. Let $k$ be a temperate weight function. Given $1 \leq p \leq \infty$, we denote by $\mathcal{B}_{p, k}$ the set of all tempered distributions $u \in \mathcal{S}\left(\mathbb{R}^{N}\right)^{\prime}$ such that the Fourier transform $\widehat{u}$ is a function and the norm

$$
\|u\|_{p, k}:=\left(\frac{1}{(2 \pi)^{N}} \int_{\mathbb{R}^{N}}|k(\xi) \widehat{u}(\xi)|^{p} d \xi\right)^{\frac{1}{p}}
$$

is finite.

When $p=\infty$, the norm $\|u\|_{p, k}$ shall be interpreted as the supremum of $|k(\xi) \widehat{u}(\xi)|$ in $\mathbb{R}^{N}$.

The space $\mathcal{B}_{p, k}$ is a Banach space with the norm $\|\cdot\|_{p, k}$.

Example 2.1.3. In case the function $k$ is given by $\widetilde{P}$ (for some polynomial $P$ ) and $p=2$, according to Parseval's formula we have

$$
\|u\|_{2, \widetilde{P}}=\left(\sum_{\alpha}\left\|P^{(\alpha)}(D) u\right\|_{2, \mathbb{R}^{N}}^{2}\right)^{\frac{1}{2}} .
$$

If $k(\xi)=\left(1+|\xi|^{2}\right)^{\frac{s}{2}}$ for some real number $s$ and $p=2$ we recover the well known Sobolev spaces $\mathcal{H}^{s}$. 
Let $\Omega$ be an open subset in $\mathbb{R}^{N}$. We denote $\mathcal{B}_{p, k}^{\text {loc }}(\Omega)$ the space of those distributions $u \in \mathcal{D}(\Omega)^{\prime}$ such that for all $\phi \in \mathcal{D}(\Omega)$, $\phi u \in \mathcal{B}_{p, k}$. Let $\left\{K_{n}\right\}_{n \in \mathbb{N}}$ be a compact exhaustion of $\Omega$ and take a sequence $\left\{\phi_{n}\right\}_{n \in \mathbb{N}}$ in $\mathcal{D}(\Omega)$ such that $\phi_{n}=1$ on $K_{n}$ and $\operatorname{supp} \phi_{n} \subset K_{n+1}$. Then, $\mathcal{B}_{p, k}^{\text {loc }}(\Omega)$ is a Fréchet space with the seminorms $u \rightarrow\left\|u \phi_{n}\right\|_{p, k}$.

Take $k=1$ and $p=2$. Observe that $\mathcal{B}_{2,1}^{\text {loc }}(\Omega)$ is the space of the functions locally in $\mathcal{L}^{2}(\Omega)$, that is, $\mathcal{L}_{\text {loc }}^{2}(\Omega)$.

The following results are useful to pass results involving spaces $\mathcal{B}_{p, k}^{\text {loc }}(\Omega)$ to statements in a classical form. See [33, II, Theorem 10.1.25 and Theorem 11.1.8].

Theorem 2.1.4. Let $j \in \mathbb{N}_{0}$ be given. The space $\mathcal{B}_{p, k}^{\text {loc }}(\Omega)$ is included in the space $\mathcal{C}^{j}(\Omega)$ of all functions $j$ times differentiable with continuous derivatives if, and only if,

$$
\frac{(1+|\xi|)^{j}}{k(\xi)} \in \mathcal{L}^{p^{\prime}} \text {, where } \frac{1}{p}+\frac{1}{p^{\prime}}=1 \text {. }
$$

Definition 2.1.5. A polynomial $P$ is called hypoelliptic if the associated differential operator $P(D)$ has the following regularity property: if $u \in$ $\mathcal{D}(\Omega)^{\prime}$ verifies that $P(D) u \in \mathcal{C}^{\infty}(\Omega)$ then $u \in \mathcal{C}^{\infty}(\Omega)$.

A complete study of this class of polynomials is given in the Chapter XI of [33, II]. The following property is useful: a polynomial $P$ is hypoelliptic if and only if there exist positive constants $c, C>0$ such that

$$
\frac{\left|P^{(\alpha)}(\xi)\right|}{|P(\xi)|} \leq C|\xi|^{-|\alpha| c}
$$

if $\xi \in \mathbb{R}^{N}$ and $|\xi|$ is large enough. We refer this condition as Condition $\mathrm{Ilb}$ of [33, II, Theorem 11.1.3].

From now on, we will suppose that the hypoelliptic polynomials in our results are non constant. Otherwise, the results will be trivial.

Theorem 2.1.6. Let $P$ be a hypoelliptic polynomial and let $u \in \mathcal{D}(\Omega)^{\prime}$ be given. If $P(D) u \in \mathcal{B}_{p, k}^{\text {loc }}(\Omega)$, then $u \in \mathcal{B}_{p, \widetilde{P} k}^{\text {loc }}(\Omega)$.

Proposition 2.1.7. Let $P$ be a hypoelliptic polynomial. Then, $\bigcap_{j \in \mathbb{N}_{0}} \mathcal{B}_{p, \widetilde{P}^{j}}^{l o c}(\Omega)=$ $\mathcal{C}^{\infty}(\Omega)$ as Fréchet spaces.

Proof. Note that $\bigcap_{j \in \mathbb{N}_{0}} \mathcal{B}_{p, \widetilde{P}^{j}}^{\text {loc }}(\Omega)$ is a Fréchet space. In order to prove $\bigcap_{j \in \mathbb{N}_{0}} \mathcal{B}_{p, \widetilde{P}^{j}}^{l o c}(\Omega) \subset \mathcal{C}^{\infty}(\Omega)$ we use Theorem 2.1.4. Let us denote

$$
\widetilde{P}^{\prime}(\xi)=\left(\sum_{\alpha \neq 0}\left|P^{(\alpha)}(\xi)\right|^{2}\right)^{\frac{1}{2}}
$$


It follows from condition IIb of [33, II, Theorem 11.1.3] that there are $c, C>$ 0 positive constants such that for all $\xi \in \mathbb{R}^{N}$,

$$
(1+|\xi|)^{c} \leq C \frac{\widetilde{P}(\xi)}{\widetilde{P}^{\prime}(\xi)} .
$$

Note that $\widetilde{P}, \widetilde{P}^{\prime}>0$. In fact, in virtue of Taylor's Theorem we have $\widetilde{P}\left(\xi_{0}\right)=$ 0 for some $\xi_{0} \in \mathbb{R}^{N}$ (respect. $\widetilde{P}^{\prime}\left(\xi_{0}\right)=0$ for some $\xi_{0} \in \mathbb{R}^{N}$ ) if and only if $P=0$ (respect. $P$ is constant). Then for all $j \in \mathbb{N}_{0}$,

$$
\frac{1}{\widetilde{P}(\xi)^{j}} \leq C^{j} \frac{1}{\widetilde{P}^{\prime}(\xi)^{j}(1+|\xi|)^{c j}} .
$$

As a consequence, given $m \in \mathbb{N}_{0}$ there is $j \in \mathbb{N}_{0}$ such that

$$
\frac{(1+|\xi|)^{m}}{\widetilde{P}(\xi)^{j}} \in \mathcal{L}^{p^{\prime}}
$$

Therefore, given $m \in \mathbb{N}_{0}$ there is $j \in \mathbb{N}_{0}$ such that

$$
\mathcal{B}_{p, \widetilde{P}^{j}}^{\text {loc }}(\Omega) \subset \mathcal{C}^{m}(\Omega)
$$

To conclude, we apply the Closed Graph Theorem.

Let $P$ be a polynomial and let $\Omega$ be an open subset of $\mathbb{R}^{N}$. Consider the space

$$
\mathcal{L}_{P}(\Omega)=\left\{f \in \mathcal{L}_{\text {loc }}^{1}(\Omega): \forall j \in \mathbb{N}_{0}, P^{j}(D) f \in \mathcal{L}_{\text {loc }}^{1}(\Omega)\right\} .
$$

Denote $\||f|\|_{L, j}:=\sup _{0 \leq k \leq j}\left\|P^{k}(D) f\right\|_{1, L}$ and endow $\mathcal{L}_{P}(\Omega)$ with the fundamental system of seminorms $\left\{\left.|||\cdot|\right|_{L, j}\right\}_{L \subset \subset \Omega, j \in \mathbb{N}_{0}}$. Then $\mathcal{L}_{P}(\Omega)$ is a Fréchet space. In fact, let $\left\{f_{n}\right\}$ be a Cauchy sequence in $\mathcal{L}_{P}(\Omega)$. Then, for each $j \in \mathbb{N}_{0}$, there is $g_{j} \in \mathcal{L}_{\text {loc }}^{1}(\Omega)$ such that $\left\{P^{j}(D) f_{n}\right\}$ converges to $g_{j}$ in $\mathcal{L}_{\text {loc }}^{1}(\Omega)$. Since $P(D)$ is continuous on $\mathcal{D}^{\prime}(\Omega)$ it follows that $g_{j}=P^{j}(D) g_{0}$. Hence, $g_{0} \in \mathcal{L}_{P}(\Omega)$ and $\left\{f_{n}\right\}$ converges to $g_{0}$ in $\mathcal{L}_{P}(\Omega)$.

Lemma 2.1.8. If $P$ is hypoelliptic, then $\mathcal{L}_{P}(\Omega)=\mathcal{C}^{\infty}(\Omega)$ as Fréchet spaces. As a consequence, for each $m \in \mathbb{N}_{0}$ and for each $K$ compact subset in $\Omega$ there are $a$ constant $C>0$, a natural number $j \in \mathbb{N}_{0}$ and a compact subset $L$ in $\Omega$ such that for all $f \in \mathcal{C}^{\infty}(\Omega)$,

$$
\sup _{|\alpha| \leq m} \sup _{x \in K}\left|f^{(\alpha)}(x)\right| \leq C \sup _{0 \leq k \leq j}\left\|P^{k}(D) f\right\|_{1, L} .
$$


Proof. Let $f \in \mathcal{L}_{P}(\Omega)$ be given and fix $j \in \mathbb{N}_{0}$. Note that the Fourier Transform of a function in $\mathcal{L}^{1}$ in bounded. Then $P(D)^{j} f \in \mathcal{L}_{\text {loc }}^{1}(\Omega) \subset$ $\mathcal{B}_{\infty, 1}^{\text {loc }}(\Omega)$ and in view of Theorem 2.1.6 we have $f \in \mathcal{B}_{\infty, \widetilde{P}^{j}}^{\text {loc }}(\Omega)$. Hence, by Proposition 2.1.7, $f \in \bigcap_{j \in \mathbb{N}_{0}} \mathcal{B}_{\infty, \widetilde{P}^{j}}^{\text {loc }}(\Omega)=\mathcal{C}^{\infty}(\Omega)$. Using the Closed Graph Theorem it follows the inclusion $\mathcal{L}_{P}(\Omega) \hookrightarrow \mathcal{C}^{\infty}(\Omega)$ is continuous.

Corollary 2.1.9. Suppose P hypoelliptic. On $\mathcal{E}_{P, *}(\Omega)$ we can replace the seminorms

$$
\|f\|_{K, \lambda}=\sup _{j \in \mathbb{N}_{0}}\left\|P^{j}(D) f\right\|_{2, K} \exp \left(-\lambda \varphi^{*}\left(\frac{j}{\lambda}\right)\right),
$$

by the seminorms

$$
\|f\|_{K, \lambda}^{p}=\sup _{j \in \mathbb{N}_{0}}\left\|P^{j}(D) f\right\|_{p, K} \exp \left(-\lambda \varphi^{*}\left(\frac{j}{\lambda}\right)\right), \quad p \geq 1,
$$

and also by the seminorms

$$
\|f\|_{K, \lambda}^{\infty}=\sup _{j \in \mathbb{N}_{0}} \sup _{x \in K}\left|P^{j}(D) f(x)\right| \exp \left(-\lambda \varphi^{*}\left(\frac{j}{\lambda}\right)\right) .
$$

Proof. Fix $1 \leq p<\infty$. We will prove that the fundamental systems of seminorms given by $\|\cdot\|_{K, \lambda}^{p}$ is equivalent to the system of seminorms given by $\|\cdot\|_{K, \lambda}^{\infty}$.

In view of the previous Lemma, for each $K$ compact subset in $\Omega$ there are a constant $C>0$, a natural number $j \in \mathbb{N}_{0}$ and $L$ compact subset in $\Omega$ such that for all $f \in \mathcal{C}^{\infty}(\Omega)$,

$$
\sup _{x \in K}|f(x)| \leq C \sup _{0 \leq k \leq j}\left\|P^{k}(D) f\right\|_{1, L} .
$$

Fix $l \in \mathbb{N}_{0}$. Applying this inequality to the function $P^{l}(D) f$ we have

$$
\sup _{x \in K}\left|P^{l}(D) f(x)\right| \leq C \sup _{0 \leq k \leq j}\left\|P^{k+l}(D) f\right\|_{1, L}
$$

for all $l \in \mathbb{N}_{0}$ and for all $f \in \mathcal{C}^{\infty}(\Omega)$. Now, proceeding as in Lemma 1.0.10 we conclude that for each compact subset $K$ in $\Omega$ and $\lambda>0$ there are $L$ compact subset and a positive constant $C^{\prime}>0$ depending on $K$ and $\lambda$ such that $\|f\|_{K, \lambda}^{\infty} \leq C^{\prime}\|f\|_{L, \mu}^{1}$ in view of Lemma 1.0.4. Moreover, the Hölder's inequality guarantees that $\|f\|_{K, \lambda}^{1} \leq C^{\prime \prime}\|f\|_{K, \lambda}^{p}$ for some positive constants $C^{\prime \prime}>0$.

Obviously, $\|f\|_{K, \lambda}^{p} \leq C^{\prime \prime \prime}\|f\|_{K, \lambda}^{\infty}$ for some positive constant $C^{\prime \prime \prime}>0$ holds. 
Assume that $P$ is hypoelliptic. Inspired by Lemma 2.1.8 and Corollary 2.1.9 we study the nuclearity of the spaces $\mathcal{E}_{P,(\omega)}(\Omega)$ and $\mathcal{E}_{P,\{\omega\}}(\Omega)$.

Definition 2.1.10. A locally convex space $E$ is said to be nuclear, if for each absolutely convex zero neighborhood $U$ in $E$ there exists an absolutely convex zero neighborhood $V$ and a measure $\mu$ on the polar $\sigma^{*}$-compact $V^{\circ}$, so that

$$
\|x\|_{U} \leq \int_{V^{\circ}}|y(x)| d \mu(y), \text { for all } x \in E .
$$

A projective limit of nuclear spaces is nuclear. The nuclearity is also an hereditary property under countable inductive limits. In order to see that an inductive limit $\operatorname{ind}_{n} X_{n}$ is nuclear it is enough to prove that for all $n$ there exists $m>n$ such that the inclusion $X_{n} \hookrightarrow X_{m}$ is a nuclear map. The composition of two absolutely summing maps is nuclear. Hence in order to prove that an inductive limit is $\operatorname{ind}_{n} X_{n}$ nuclear it is enough to prove that for all $n$ there exists $m>n$ such that the inclusion $X_{n} \hookrightarrow X_{m}$ is absolutely summing.

Let $E$ and $F$ be Banach spaces. Denote by $B_{E}$ the unit ball of $E$. Recall that a sufficient condition in order that a linear map $T: E \rightarrow F$ be absolutely summing is that there is a positive measure $\mu$ on $B_{E}^{\circ}$ such that

$$
\|T x\| \leq \int_{B_{E}^{\circ}}|y(x)| d \mu(y), \text { for all } x \in E .
$$

See the Chapter 28 of [54] and the books [34] and [41, II] for more details.

Theorem 2.1.11. Suppose $P$ is hypoelliptic. Then, the spaces $\mathcal{E}_{P,(\omega)}(\Omega)$ and $\mathcal{E}_{P,\{\omega\}}(\Omega)$ are nuclear.

Proof. Beurling case:

First, observe that Lemma 1.0.3 allows replace the seminorms

$$
\|f\|_{K, \lambda}^{p}=\sup _{j \in \mathbb{N}_{0}}\left\|P^{j}(D) f\right\|_{p, K} \exp \left(-\lambda \varphi^{*}\left(\frac{j}{\lambda}\right)\right), \quad 1 \leq p \leq \infty,
$$

by the seminorms

$$
\sum_{j \in \mathbb{N}_{0}}\left\|P^{j}(D) f\right\|_{p, K} \exp \left(-\lambda \varphi^{*}\left(\frac{j}{\lambda}\right)\right) .
$$

In fact, this Lemma and the condition $(\alpha)$ of weight function give a natural number $L$ and $y_{0}>0$ such that

$$
\varphi^{*}(y)-y \geq L \varphi^{*}\left(\frac{y}{L}\right)-L \text { for each } y \geq y_{0} .
$$


Take $y=\frac{j}{\lambda}$. For $j$ large enough, the last inequality implies

$$
\exp \left(-\lambda \varphi^{*}\left(\frac{j}{\lambda}\right)\right) \leq \exp (L \lambda) \exp (-j) \exp \left(-L \lambda \varphi^{*}\left(\frac{j}{L \lambda}\right)\right) \text {. }
$$

Thus, fixed $K \subset \subset \Omega$ and $\lambda>0$ there is a constant $C>0$ such that for all $f \in \mathcal{E}_{P,(\omega)}(\Omega)$

$$
\|f\|_{K, \lambda}^{p} \leq \sum_{j \in \mathbb{N}_{0}}\left\|P^{j}(D) f\right\|_{p, K} \exp \left(-\lambda \varphi^{*}\left(\frac{j}{\lambda}\right)\right) \leq C\|f\|_{K, L \lambda}^{p} .
$$

We consider $\mathcal{E}_{P,(\omega)}(\Omega)$ endowed with the seminorms

$$
\sum_{j \in \mathbb{N}_{0}} \int_{K}\left|P^{j}(D) f(x)\right| d x \exp \left(-\lambda \varphi^{*}\left(\frac{j}{\lambda}\right)\right) .
$$

Fix $\lambda>0$ and $K$ compact subset. By the inequality (2.2) we have

$$
\begin{gathered}
\sum_{j \in \mathbb{N}_{0}} \int_{K}\left|P^{j}(D) f(x)\right| d x \exp \left(-\lambda \varphi^{*}\left(\frac{j}{\lambda}\right)\right) \leq \\
C \sum_{j \in \mathbb{N}_{0}} \int_{K}\left|P^{j}(D) f(x)\right| d x \exp \left(-L \lambda \varphi^{*}\left(\frac{j}{L \lambda}\right)\right) \exp (-j)
\end{gathered}
$$

for some positive constant $C>0$. Define

$$
\Delta_{j}: K \rightarrow\left(\mathcal{E}_{P,(\omega)}(\Omega)^{\prime}, \sigma\left(\mathcal{E}_{P,(\omega)}(\Omega)^{\prime}, \mathcal{E}_{P,(\omega)}(\Omega)\right)\right)
$$

such that

$$
\Delta_{j}(x)[f]:=P^{j}(D) f(x) \exp \left(-L \lambda \varphi^{*}\left(\frac{j}{L \lambda}\right)\right)
$$

Then,

$$
\left|\Delta_{j}(x)[f]\right| \leq\|f\|_{K, L \lambda}^{\infty} .
$$

Hence, $\Delta_{j}(x) \in \mathcal{E}_{P,(\omega)}(\Omega)^{\prime}$ and then $\Delta_{j}$ is a well defined and continuous map. Moreover $\Delta_{j}(K) \subseteq V^{\circ}$ where $V$ is the absolutely convex zero neighborhood defined by

$$
V:=\left\{f \in \mathcal{E}_{P,(\omega)}(\Omega) \text { such that }\|f\|_{K, L \lambda}^{\infty} \leq 1\right\} .
$$

Now, consider the following map

$$
\mu_{j}: \mathcal{C}\left(V^{\circ}\right) \rightarrow \mathbb{R}, \mu_{j}(g):=C \int_{K} g\left(\Delta_{j}(x)\right) \exp (-j) d x
$$


Denote $m(K)$ the Lebesgue measure of the compact $K$. If $g: V^{\circ} \rightarrow \mathbb{R}$ is a continuous function on $V^{\circ}$, it is clear that

$$
\left|\mu_{j}(g)\right| \leq C \exp (-j) m(K) \sup _{f \in V^{\circ}}|g(f)| .
$$

This fact implies that $\mu_{j}$ is a continuous linear map which is positive, i.e, $\mu_{j}(g) \geq 0$ whenever $g \geq 0$. So, $\mu_{j}$ defines a measure on $\left(V^{\circ}, \sigma^{*}\right)$. We consider now

$$
\mu:=\sum_{j \in \mathbb{N}_{0}} \mu_{j}
$$

which is a measure on $\left(V^{\circ}, \sigma^{*}\right)$. Then,

$$
\begin{aligned}
& \sum_{j \in \mathbb{N}_{0}} \int_{K}\left|P^{j}(D) f(x)\right| d x \exp \left(-\lambda \varphi^{*}\left(\frac{j}{\lambda}\right)\right) \leq \\
& C \sum_{j \in \mathbb{N}_{0}} \int_{K}\left|P^{j}(D) f(x)\right| d x \exp \left(-L \lambda \varphi^{*}\left(\frac{j}{L \lambda}\right)\right) \exp (-j) \leq \\
& \leq C \sum_{j \in \mathbb{N}_{0}} \int_{K}\left|\Delta_{j}(x)[f]\right| d x \exp (-j)=\sum_{j \in \mathbb{N}_{0}} \int_{V^{\circ}}|y(f)| d \mu_{j}(y) \\
& =\int_{V^{\circ}}|y(f)| d \mu(y) \text {. }
\end{aligned}
$$

and the nuclearity in the Beurling case follows.

\section{Roumieu case:}

To see that

$$
\mathcal{E}_{P,\{\omega\}}(\Omega):=\underset{K \subset \subset \Omega}{\stackrel{\text { proj }}{\longleftarrow}} \underset{\lambda>0}{\text { ind }} \mathcal{E}_{P, \omega}^{\lambda}(K)
$$

is nuclear it is enough to see that

$$
\underset{n}{\stackrel{\text { ind }}{\longrightarrow}} \mathcal{E}_{P, \omega}^{\frac{1}{n}}(K)
$$

is nuclear for each compact subset $K$. We will see that the map

$$
\mathcal{E}_{P, \omega}^{\frac{1}{n}}(K) \hookrightarrow \mathcal{E}_{P, \omega}^{\frac{1}{n L}}(K)
$$

is absolutely summing. $\mathcal{E}_{P, \omega}^{\frac{1}{n}}(K)$ and $\mathcal{E}_{P, \omega}^{\frac{1}{n}}(K)$ are Banach spaces endowed with the norms $\|\cdot\|_{K, \frac{1}{L n}}^{\infty}$ and $\|\cdot\|_{K, \frac{1}{n}}^{\infty}$, respectively. Note that the inequality (2.2) applied to $\lambda=\frac{1}{n L}$ gives, for $j$ large enough,

$$
\exp \left(-\frac{1}{n L} \varphi^{*}(L n j)\right) \leq \exp \left(\frac{1}{n}\right) \exp (-j) \exp \left(-\frac{1}{n} \varphi^{*}(n j)\right) .
$$


Then, for some constant $C>0$, we have

$$
\begin{aligned}
\|f\|_{K, \frac{1}{L n}}^{\infty} & \leq \sum_{j \in \mathbb{N}_{0}}\left\|P^{j}(D) f\right\|_{p, K} \exp \left(-\frac{1}{L n} \varphi^{*}(L n j)\right) \\
& \leq C \exp \left(\frac{1}{n}\right) \sum_{j \in \mathbb{N}_{0}}\left\|P^{j}(D) f\right\|_{p, K} \exp \left(-\frac{1}{n} \varphi^{*}(n j)\right) \exp (-j) .
\end{aligned}
$$

Define

$$
\Delta_{j}: K \rightarrow\left(\mathcal{E}_{P, \omega}^{\frac{1}{n}}(K)^{\prime}, \sigma\left(\mathcal{E}_{P, \omega}^{\frac{1}{n}}(K)^{\prime}, \mathcal{E}_{P, \omega}^{\frac{1}{n}}(K)\right)\right)
$$

such that

$$
\Delta_{j}(x)[f]:=P^{j}(D) f(x) \exp \left(-\frac{1}{n} \varphi^{*}(n j)\right)
$$

Denote by $U$ the unit ball of $\mathcal{E}_{P, \omega}^{\frac{1}{n}}(K)$. Proceeding as in the Beurling case we can define a measure on $U^{\circ}$ such that

$$
\|f\|_{K, \frac{1}{L n}}^{\infty} \leq \int_{U^{\circ}}|y(f)| d \mu(y)
$$

\subsection{The completeness of $\mathcal{E}_{P, \omega}(\Omega)$}

In order to extend the results by Newberger-Zielezny [57] we need to apply the Closed Graph Theorem and the Grothendieck's Factorization Theorem. So, it is important to know whether the spaces $\mathcal{E}_{P, *}(\Omega)$ are complete or not. In this section we show that the spaces $\mathcal{E}_{P, *}(\Omega)$ are not necessarily complete spaces. In fact, completeness is characterized in terms of the hypoellipticity of the polynomial $P$. Moreover, in case completeness fails, a finer topology on $\mathcal{E}_{P, *}(\Omega)$ is introduced so that the space becomes complete. This topology will be considered in Theorem 3.1.2. In spite of the importance of the completeness when dealing with functional analytic tools, as far as we know this is the first time that the completeness of these spaces is discussed.

We start with an example:

Example 2.2.1. The space $\mathcal{E}_{P,(\omega)}\left(\mathbb{R}^{2}\right)$ is not complete for the polynomial $P(x, y)=x$. 
Proof. Let $\left\{\rho_{m}\right\}_{m \in \mathbb{N}}$ be a regularizing sequence on the real line and take a function $g(y) \in \mathcal{C}(\mathbb{R}) \backslash \mathcal{C}^{\infty}(\mathbb{R})$. We define $f_{m}(x, y):=\left(\rho_{m} * g\right)(y), m \in \mathbb{N}$, a sequence of functions in $\mathbb{R}^{2}$. It is well known that $f_{m} \in \mathcal{C}^{\infty}\left(\mathbb{R}^{2}\right)$ and, for $K_{n}=[-n, n]^{2}$,

$$
\begin{aligned}
q_{K_{n}, n}\left(f_{m}\right) & =\left\|\frac{\partial^{0}}{\partial x^{0}} f_{m}(x, y)\right\|_{2, K_{n}} \exp \left(-n \varphi^{*}\left(\frac{0}{n}\right)\right) \\
& =(2 n)^{\frac{1}{2}}\left\|\left(\rho_{m} * g\right)(y)\right\|_{2,[-n, n]}<+\infty .
\end{aligned}
$$

Therefore, $\left\{f_{m}\right\}_{m \in \mathbb{N}}$ is a sequence in $\mathcal{E}_{P,(\omega)}\left(\mathbb{R}^{2}\right)$. Moreover, since for each $n \in \mathbb{N}$ we have,

$$
q_{K_{n}, n}\left(f_{m}-f_{l}\right) \leq 2 n \sup _{[-n, n]}\left|\left(\rho_{m} * g\right)(y)-\left(\rho_{l} * g\right)(y)\right|,
$$

and $\left\{\rho_{m} * g\right\}_{m \in \mathbb{N}}$ converges uniformly to $g$ on the compact subsets of the real line, it is clear that $\left\{f_{m}\right\}_{m \in \mathbb{N}}$ is a Cauchy sequence in $\mathcal{E}_{P,(\omega)}\left(\mathbb{R}^{2}\right)$. However, $\left\{f_{m}\right\}_{m \in \mathbb{N}}$ is not convergent. Otherwise, let $f$ denote the corresponding limit. Then $\left\{f_{m}(x, y)\right\}_{m \in \mathbb{N}}$ converges to $f(x, y)$ for all $(x, y) \in \mathbb{R}^{2}$, from where it follows that $f(x, y)=g(y)$. This is a contradiction because $g$ is not a $\mathcal{C}^{\infty}$ function.

The previous example shows that in order to have completeness for the space $\mathcal{E}_{P,(\omega)}(\Omega)$ it is necessary to obtain $\mathcal{C}^{\infty}(\Omega)$ functions from Cauchy sequences in $\mathcal{E}_{P,(\omega)}(\Omega)$. According to [33, II, 11.1.5], if the solutions of $P(D) u=0, u \in \mathcal{B}_{p, k}^{\text {loc }}(\Omega)$, are all in $C^{\infty}(\Omega)$ then $P(D)$ has to be hypoelliptic. Therefore, if $P(D)$ is not hypoelliptic we can find $u \in \mathcal{B}_{p, k}^{\text {loc }}(\Omega) \backslash C^{\infty}(\Omega)$ such that $P(D) u=0$. A suitable election of $p$ and $k$ (see Theorem 2.1.4 and Proposition 2.1.7) allows us to assume that this $u$ belonging to the kernel of $P(D)$ is a continuous function or even, a $\mathcal{C}^{m}$-function. As a consequence, we need the hypoellipticity of $P$ in order that $\mathcal{E}_{P, *}(\Omega)$ becomes complete.

Proposition 2.2.2. Let $\Omega$ be an open subset of $\mathbb{R}^{N}$. If the space $\mathcal{E}_{P, *}(\Omega)$ is complete, then $P$ is hypoelliptic.

Proof. Proceeding by contradiction we assume that $P$ is not hypoelliptic. We first analyze the case that $\Omega=\mathbb{R}^{N}$. Since $P$ is not hypoelliptic, there exists a continuous function

$$
u \in C\left(\mathbb{R}^{N}\right) \backslash C^{\infty}\left(\mathbb{R}^{N}\right) \text { such that } P(D) u=0 .
$$

Beurling case. We take a regularizing sequence $\left\{\rho_{n}\right\}$ with supp $\rho_{n}=\overline{B\left(0, \frac{1}{n}\right)}$ and we show that $\left\{u * \rho_{n}\right\}$ is a Cauchy sequence in $\mathcal{E}_{P,(\omega)}\left(\mathbb{R}^{N}\right)$ which is not convergent. 
It is clear that $u * \rho_{n} \in \mathcal{C}^{\infty}\left(\mathbb{R}^{N}\right)$ for all $n \in \mathbb{N}$. Moreover, $P(D)(u *$ $\left.\rho_{n}\right)=P(D) u * \rho_{n}=0$. As a consequence, $P^{j}(D)\left(u * \rho_{n}\right)=0$ if $j \neq 0$. Therefore,

$$
\left\|u * \rho_{n}\right\|_{K, \lambda} \leq(m(K))^{\frac{1}{2}} \sup _{x \in K}\left|u * \rho_{n}(x)\right|<+\infty,
$$

for all $K \subset \subset \mathbb{R}^{N}$ and for all $\lambda>0$, i.e, $u * \rho_{n} \in \mathcal{E}_{P,(\omega)}\left(\mathbb{R}^{N}\right)$ for each $n \in \mathbb{N}$. In a similar way,

$$
\left\|u * \rho_{n}-u * \rho_{l}\right\|_{K, \lambda} \leq(m(K))^{\frac{1}{2}} \sup _{x \in K}\left|u *\left(\rho_{n}-\rho_{l}\right)(x)\right|
$$

which implies that $\left\{u * \rho_{n}\right\}$ is a Cauchy sequence in $\mathcal{E}_{P,(\omega)}\left(\mathbb{R}^{N}\right)$. If $\{u *$ $\left.\rho_{n}\right\}$ converges to $f \in \mathcal{E}_{P,(\omega)}\left(\mathbb{R}^{N}\right)$, then $\left\{u * \rho_{n}\right\}$ converges to $f$ uniformly on the compact sets, hence $f=u$. This is a contradiction since $u$ is not infinitely differentiable.

Roumieu case. The sequence $\left\{u * \rho_{n}\right\}$ constructed in the Beurling case is a Cauchy sequence in $\mathcal{E}_{P,\{\omega\}}\left(\mathbb{R}^{N}\right)$ since the inclusion map

$$
\mathcal{E}_{P,(\omega)}\left(\mathbb{R}^{N}\right) \hookrightarrow \mathcal{E}_{P,\{\omega\}}\left(\mathbb{R}^{N}\right)
$$

is continuous. We see that $\left\{u * \rho_{n}\right\}$ does not have limit in $\mathcal{E}_{P,\{\omega\}}\left(\mathbb{R}^{N}\right)$. We recall that

$$
\mathcal{L}_{\text {loc }}^{2}\left(\mathbb{R}^{N}\right)=\underset{K \subset \subset \mathbb{R}^{N}}{\operatorname{proj}}\left\{f \text { mesurable }:\|f\|_{2, K}<\infty\right\} .
$$

Then, the inclusion map

$$
\mathcal{E}_{P,\{\omega\}}\left(\mathbb{R}^{N}\right) \hookrightarrow \mathcal{L}_{l o c}^{2}\left(\mathbb{R}^{N}\right)
$$

is continuous. If $\left\{u * \rho_{n}\right\}$ converges to $f$ in $\mathcal{E}_{P,\{\omega\}}\left(\mathbb{R}^{N}\right)$, then $\left\{u * \rho_{n}\right\}$ converges to $f$ in $\mathcal{L}_{\text {loc }}^{2}\left(\mathbb{R}^{N}\right)$. However, for each $K \subset \subset \mathbb{R}^{N}$

$$
\left\|u * \rho_{n}-u\right\|_{2, K} \leq(m(K))^{\frac{1}{2}} \sup _{x \in K}\left|u * \rho_{n}(x)-u(x)\right| \rightarrow 0 \text { as } n \rightarrow+\infty .
$$

Then $f=u$, which is a contradiction since $\mathrm{u}$ is not $\mathcal{C}^{\infty}$.

In the case that $\Omega$ is an arbitrary open subset of $\mathbb{R}^{N}$, we can assume (after a suitable translation if necessary) that

$$
\exists u \in C(\Omega+B(0,1)) \backslash C^{\infty}(\Omega) \text { such that } P(D) u=0 .
$$

Then the convolutions $u * \rho_{n}$ are defined on $\Omega$ and we can proceed as above. 
In order to prove the converse of Proposition 2.2.2, we introduce the following spaces. Let $\omega$ be a weight function. Given a polynomial $P$, an open set $\Omega$ of $\mathbb{R}^{N}$, a compact subset $K \subset \subset \Omega$ and $\lambda>0$, define

$$
r_{K, \lambda}(f):=\sup _{j \in \mathbb{N}_{0}}\left\|P^{j}(D) f\right\|_{1, K} \exp \left(-\lambda \varphi^{*}\left(\frac{j}{\lambda}\right)\right)
$$

and

$$
\mathcal{L}_{P, \omega}^{\lambda}(K):=\left\{f \in \mathcal{L}^{1}(K): P^{j}(D) f \in \mathcal{L}^{1}(K) \forall j \in \mathbb{N}_{0}, r_{K, \lambda}(f)<+\infty\right\} .
$$

Beurling case:

$$
\mathcal{L}_{P,(\omega)}(\Omega)=\left\{f \in \mathcal{L}_{\text {loc }}^{1}(\Omega): f \in \mathcal{L}_{P, \omega}^{\lambda}(K) \text { for each } K \subset \subset \Omega \text { and } \lambda>0\right\} .
$$

If $\left\{K_{n}\right\}_{n \in \mathbb{N}}$ is a compact exhaustion of $\Omega$ this space is endowed with the topology given by

$$
\mathcal{L}_{P,(\omega)}(\Omega)=\underbrace{\text { proj }}_{n \in \mathbb{N}} \underset{k \in \mathbb{N}}{\overleftarrow{\operatorname{proj}}} \mathcal{L}_{P, \omega}^{k}\left(K_{n}\right)=\underset{n \in \mathbb{N}}{\operatorname{proj}} \mathcal{L}_{P, \omega}^{n}\left(K_{n}\right) .
$$

This metrizable locally convex topology is defined by the fundamental system of seminorms $\left\{r_{K_{n}, n}(\cdot)\right\}_{n \in \mathbb{N}}$. Using standard arguments it follows that $\mathcal{L}_{P,(\omega)}(\Omega)$ is a Fréchet space.

Theorem 2.2.3. Let $\Omega$ be an open subset of $\mathbb{R}^{N}$. For a weight function $\omega$ and a polynomial $P, \mathcal{L}_{P,(\omega)}(\Omega)$ is a Fréchet space if it is endowed with the topology defined above.

Proof. Let $\left\{f_{k}\right\}_{k \in \mathbb{N}}$ be a Cauchy sequence in $\mathcal{L}_{P,(\omega)}(\Omega)$. We fix $n \in \mathbb{N}$ and $\varepsilon>0$, then $\exists l_{0} \in \mathbb{N}$ such that

$$
\left\|P^{j}(D)\left(f_{l}-f_{k}\right)\right\|_{1, K_{n}} \exp \left(-n \varphi^{*}\left(\frac{j}{n}\right)\right)<\varepsilon \text { if } k, l \geq l_{0},
$$

for all $j \in \mathbb{N}_{0}$.

On the other hand, for each $j \in \mathbb{N}_{0}$ there exist $\left(n_{k}\right)$ and $h_{j} \in \mathcal{L}^{1}\left(K_{n}\right)$ such that $P^{j}(D) f_{n_{k}}$ converges to $h_{j}$ a.e and $\left\|P^{j}(D)\left(f_{l}\right)-h_{j}\right\|_{1, K_{n}} \exp \left(-n \varphi^{*}\left(\frac{j}{n}\right)\right)$ converges to 0 if $l \rightarrow+\infty$. Since $P(D)$ is continuous on $\mathcal{D}(\Omega)^{\prime}$ we have $h_{j}=P^{j}(D) f \in \mathcal{L}^{1}\left(K_{n}\right)$.

In (2.4) we take $l=l_{0}, k \rightarrow+\infty$ and we have

$$
\left\|P^{j}(D)\left(f_{l_{0}}-f\right)\right\|_{1, K_{n}} \exp \left(-n \varphi^{*}\left(\frac{j}{n}\right)\right) \leq \varepsilon .
$$


In particular, for all $j \in \mathbb{N}$,

$$
\left\|P^{j}(D) f\right\|_{1, K_{n}} \exp \left(-n \varphi^{*}\left(\frac{j}{n}\right)\right) \mid \leq \varepsilon+\left\|f_{l_{0}}\right\|_{n},
$$

which shows that

$$
r_{K_{n}, n}(f)<\varepsilon+r_{K_{n}, n}\left(f_{l_{0}}\right)<+\infty
$$

and $f \in \mathcal{L}_{P,(\omega)}(\Omega)$. Moreover, from (2.4) proceeding as above we obtain $r_{K_{n}, n}\left(f_{k}-f\right) \rightarrow 0$ as $k \rightarrow \infty$ and the proof is finished.

Roumieu case:

$$
\mathcal{L}_{P,\{\omega\}}(\Omega)=\left\{f \in \mathcal{L}_{l o c}^{1}(\Omega): \forall K \subset \subset \Omega \exists \lambda>0 \text { such that } f \in \mathcal{L}_{P, \omega}^{\lambda}(K)\right\} .
$$

This space is endowed with the following locally convex topology:

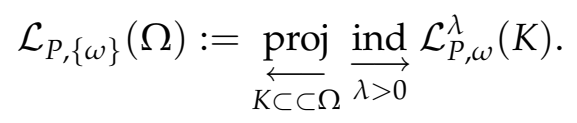

Proposition 2.2.4. The space of Roumieu type $\mathcal{L}_{P,\{\omega\}}(\Omega)$ is complete.

Proof. We fix a compact subset $K$ of $\Omega$. If suffices to prove that the countable inductive limit of Banach spaces

$$
X=\underset{n \in \mathbb{N}}{\operatorname{ind}} \mathcal{L}_{P, \omega}^{n}(K)
$$

is complete. According to a Theorem of Mujica [56] (see also [58, Corollary 8.5.22 (ii)]), we only need to check that there is a Hausdorff locally convex topology $s$ on $X$ such that the unit ball of each $\mathcal{L}_{P, \omega}^{n}(K)$ is compact in $(X, s)$. To do this it is enough to apply the Banach-Alaoglu Theorem (see [54, Theorem 23.5]): consider $E:=\prod_{j \in \mathbb{N}_{0}}\left(\mathcal{L}^{1}\left(K_{n}\right), t_{n}\right)$, where $t_{n}$ denotes the weak topology, and define $s$ as the topology induced on $X$ by the injective map

$$
X \rightarrow E, f \mapsto\left\{P^{j}(D) f\right\}_{j \in \mathbb{N}_{0}}
$$

Theorem 2.2.5. The space $\mathcal{E}_{P, *}(\Omega)$ is complete if and only if $P$ is a hypoelliptic polynomial. 
Proof. Let us assume that $P$ is hypoelliptic. In order to show that $\mathcal{E}_{P, *}(\Omega)$ is complete it suffices to prove that the spaces $\mathcal{E}_{P, *}(\Omega)$ and $\mathcal{L}_{P, *}(\Omega)$ coincide algebraically and topologically. Fix $\Omega^{\prime}$ a relatively compact open subset in $\Omega$. Let $f \in \mathcal{L}_{P, *}\left(\Omega^{\prime}\right)$ be given. Since $P(D)^{j} f \in \mathcal{L}_{\text {loc }}^{1}\left(\Omega^{\prime}\right) \subset \mathcal{B}_{\infty, 1}^{\text {loc }}\left(\Omega^{\prime}\right)$, in view of Theorem 2.1.6 we have $f \in \mathcal{B}_{\infty, \widetilde{P}^{j}}^{\text {loc }}\left(\Omega^{\prime}\right)$. Hence, by Proposition 2.1.7, $f \in \bigcap_{j \in \mathbb{N}_{0}} \mathcal{B}_{\infty, \widetilde{P}^{j}}^{\text {loc }}\left(\Omega^{\prime}\right)=\mathcal{C}^{\infty}\left(\Omega^{\prime}\right)$. Denote by $\left(K_{j}\right)_{j}$ a fundamental sequence of compact sets in $\Omega$ such that each $K_{j}$ is contained in the interior of $K_{j+1}$. Our argument above shows that the restriction maps $\mathcal{L}_{P, \omega}^{\lambda}\left(K_{j+1}\right)$ into $\mathcal{E}_{P, \omega}^{\lambda}\left(K_{j}\right)$ continuously, from where the conclusion follows.

In order to construct a complete finer locally convex topology on $\mathcal{E}_{P, *}(\Omega)$ in the general case, we fix a compact exhaustion $\left\{K_{n}\right\}$ of $\Omega$ and we consider the following system of seminorms on $\mathcal{E}_{P,(\omega)}(\Omega)$ :

$$
\left\{\|\cdot\|_{n}\right\}_{n \in \mathbb{N}} \cup\left\{p_{n}\right\}_{n \in \mathbb{N}}
$$

where

$$
\|f\|_{n}:=\|f\|_{K_{n}, n}=\sup _{j \in \mathbb{N}_{0}}\left\|P^{j}(D) f\right\|_{2, K_{n}} \exp \left(-n \varphi^{*}\left(\frac{j}{n}\right)\right)
$$

and $\left\{p_{n}\right\}_{n \in \mathbb{N}}$ is a fundamental system of seminorms of $\mathcal{E}(\Omega)$, i.e,

$$
p_{n}(f):=\sup _{|\alpha| \leq n} \sup _{x \in K_{n}}\left|f^{(\alpha)}(x)\right| .
$$

Then, $\left\{\max \left(\|\cdot\|_{n}, p_{n}\right)\right\}_{n \in \mathbb{N}}$ is a fundamental system of seminorms of a locally convex topology $\tau_{(\omega), \infty}$ on $\mathcal{E}_{P,(\omega)}(\Omega)$. The proof of the following result is standard. One can adapt the proof of Theorem 2.2.3.

Proposition 2.2.6. Let $\Omega$ be an open subset of $\mathbb{R}^{N}$. For a weight function $\omega$ and a polynomial $P$, the space $\left(\mathcal{E}_{P,(\omega)}(\Omega), \tau_{(\omega), \infty}\right)$ is a Fréchet space.

Proof. Let $\left\{f_{k}\right\}_{k \in \mathbb{N}}$ be a Cauchy sequence in $\mathcal{E}_{P,(\omega)}(\Omega)$. In particular, $\left\{f_{k}\right\}_{k \in \mathbb{N}}$ is a Cauchy sequence in $\mathcal{E}(\Omega)$, which is complete. Hence there exists $f \in \mathcal{E}(\Omega)$ such that for each $n \in \mathbb{N}, p_{n}\left(f_{k}-f\right) \rightarrow 0$ as $k \rightarrow+\infty$. Now, we see that $\left\{f_{k}\right\}_{k \in \mathbb{N}} \rightarrow f$ in $\mathcal{E}_{P,(\omega)}(\Omega)$. We only need to prove that for each $n \in \mathbb{N}$,

$$
\|f\|_{n}<+\infty
$$

and

$$
\left\|f_{k}-f\right\|_{n} \rightarrow 0 \text { as } k \rightarrow+\infty
$$


We fix $n \in \mathbb{N}$ and $\varepsilon>0$, then

$$
\exists l_{0} \in \mathbb{N} \text { such that }\left\|P^{j}(D)\left(f_{l}-f_{k}\right)\right\|_{2, K_{n}} \exp \left(-n \varphi^{*}\left(\frac{j}{n}\right)\right)<\varepsilon \text { if } k, l \geq l_{0},
$$

for all $j \in \mathbb{N}_{0}$.

On the other hand, for each $j \in \mathbb{N}_{0}$ there exist $\left(n_{k}\right)$ and $h_{j} \in \mathcal{L}^{2}\left(K_{n}\right)$ such that $P^{j}(D) f_{n_{k}}$ converges to $h_{j}$ a.e and $\left\|P^{j}(D)\left(f_{l}\right)-h_{j}\right\|_{2, K_{n}} \exp \left(-n \varphi^{*}\left(\frac{j}{n}\right)\right)$ converges to 0 if $l \rightarrow+\infty$. Since $P(D)$ is continuous in $\mathcal{E}(\Omega)$ we have $h_{j}=P^{j}(D) f$.

In (2.5) we take $l=l_{0}, k \rightarrow+\infty$ and we have

$$
\left\|P^{j}(D)\left(f_{l_{0}}-f\right)\right\|_{2, K_{n}} \exp \left(-n \varphi^{*}\left(\frac{j}{n}\right)\right) \leq \varepsilon .
$$

In particular, for all $j \in \mathbb{N}$,

$$
\left\|P^{j}(D) f\right\|_{2, K_{n}} \exp \left(-n \varphi^{*}\left(\frac{j}{n}\right)\right) \leq \varepsilon+\left\|f_{l_{0}}\right\|_{n},
$$

which shows that

$$
\|f\|_{n}<\varepsilon+\left\|f_{l_{0}}\right\|_{n}<+\infty
$$

and $f \in \mathcal{E}_{P,(\omega)}(\Omega)$. Moreover, from (2.5) proceeding as above we obtain $\left\|f_{k}-f\right\|_{n} \rightarrow 0$ as $k \rightarrow \infty$ and the proof is finished.

In the Roumieu case, we consider the following topology: for $n \in \mathbb{N}$ and $K \subset \subset \Omega$, we endow $\mathcal{E}_{P, \omega}^{\frac{1}{n}}(K)$ with the fundamental system of seminorms

$$
\left\{\max \left(\|\cdot\|_{K, \frac{1}{n}}, p_{m}\right)\right\}_{m \in \mathbb{N}} .
$$

It is easy to see that $\mathcal{E}_{P, \omega}^{\frac{1}{n}}(K)$ is a Fréchet space. The topology $\tau_{\{\omega\}, \infty}$ on $\mathcal{E}_{P,\{\omega\}}(\Omega)$ is defined by

$$
\left(\mathcal{E}_{P,\{\omega\}}(\Omega), \tau_{\{\omega\}, \infty}\right)=\underset{K \subset \subset \Omega}{\operatorname{proj}} \underset{n \in \mathbb{N}}{\text { ind }} \mathcal{E}_{P, \omega}^{\frac{1}{n}}(K) .
$$

The space $\underset{n \in \mathbb{N}}{\operatorname{ind}} \mathcal{E}_{P, \omega}^{\frac{1}{n}}(K)$ is an (LF)-space, i.e, a countable inductive limit of Fréchet spaces.

Proposition 2.2.7. Let $\Omega$ be an open subset of $\mathbb{R}^{N}$. For a weight function $\omega$ and a polynomial $P$, the space $\left(\mathcal{E}_{P,\{\omega\}}(\Omega), \tau_{\{\omega\}, \infty}\right)$ is complete.

The proof requires the following result for (LF)-spaces. 
Definition 2.2.8. Let $X=\underset{n \in \mathbb{N}}{\operatorname{ind}} X_{n}$ be an (LF)-space. $X$ is called boundedly stable if on each set which is bounded in some $X_{n}$ all but finitely many of the step topologies coincide.

Next theorem, due to Wengenroth, follows from Theorem 6.4 (page 112) and Corollary 6.4 (page 113) of [64]:

Theorem 2.2.9. Let $X=\underset{n \in \mathbb{N}}{\operatorname{ind}} X_{n}$ be an (LF)-space and $\left\{\|\cdot\|_{n, m}\right\}_{m \in \mathbb{N}}$ a fundamental system of seminorms of $X_{n}$. If $X$ is boundedly stable and satisfies the condition $\left(P 3^{*}\right)$, i.e,

$$
\begin{gathered}
\forall n \exists l \geq n \forall m \geq l \exists N \in \mathbb{N} \forall M \in \mathbb{N} \exists K \in \mathbb{N}, S>0 \forall x \in X_{n} \\
\|x\|_{l, M} \leq S\left(\|x\|_{m, K}+\|x\|_{n, N}\right),
\end{gathered}
$$

then $X$ is complete.

Proof of Proposition 2.2.7: It is enough to show that for each $K \subset \subset \Omega$, the space $X=\underset{n \in \mathbb{N}}{\underset{\text { ind }}{\longrightarrow}} \mathcal{E}_{P, \omega}^{\frac{1}{n}}(K)$ is complete. We denote $X_{n}=\mathcal{E}_{P, \omega}^{\frac{1}{n}}(K)$ and $\left\{\|\cdot\|_{n, m}\right\}_{m \in \mathbb{N}}=\left\{\max \left(\|\cdot\|_{\frac{1}{n}}, p_{m}\right)\right\}_{m \in \mathbb{N}}$. To see that $X$ is complete, we show that $X$ verifies the hypothesis of Theorem 2.2.9, i.e, $X$ is boundedly stable and satisfies the condition $\left(\mathrm{P}^{*}\right)$. We apply Lemma 1.0.3 and the condition $(\alpha)$ of weight function to get a natural number $L$ and $y_{0}>0$ such that

$$
\varphi^{*}(y)-y \geq L \varphi^{*}\left(\frac{y}{L}\right)-L \text { for each } y \geq y_{0} .
$$

Take $y=\frac{j m}{\lambda}$. The last inequality implies

$$
\frac{\exp \left(-\lambda \varphi^{*}\left(\frac{m j}{\lambda}\right)\right)}{\exp \left(-\lambda L \varphi^{*}\left(\frac{m j}{\lambda L}\right)\right)} \leq \exp (L \lambda-j m) \longrightarrow 0 \text { if } j \rightarrow+\infty .
$$

To see that $X$ is boundedly stable it suffices to prove that any bounded set $\mathcal{B}$ in $X_{n}$ is relatively compact in $X_{n L}$. Since $\mathcal{E}(\Omega)$ is a Montel space, we only need to show that if $\left\{f_{k}\right\}_{k \in \mathbb{N}}$ is a bounded sequence in $X_{n}$ and converges to 0 in $\mathcal{E}(\Omega)$, then

$$
\left\{f_{k}\right\}_{k \in \mathbb{N}} \text { converges to } 0 \text { in } X_{n L} \text {, i.e, }\left\|f_{k}\right\|_{K, \frac{1}{n L}} \longrightarrow 0 \text { si } k \rightarrow+\infty \text {. }
$$

Since $\left\{f_{k}\right\}_{k \in \mathbb{N}}$ is a bounded sequence in $X_{n}$, there exists a constant $C>0$ such that for all $k \in \mathbb{N},\left\|f_{k}\right\|_{K, \frac{1}{n}} \leq C$. Let $\eta>0$, in view of (2.6) there exists $j_{0} \in \mathbb{N}_{0}$ such that

$$
\left\|P^{j}(D)\left(f_{k}\right)\right\|_{2, K} \exp \left(-\frac{1}{L n} \varphi^{*}(j n L)\right) \leq
$$




$$
\leq \eta\left(\sup _{i \in \mathbb{N}_{0}}\left\|P^{i}(D)\left(f_{k}\right)\right\|_{2, K} \exp \left(-\frac{1}{n} \varphi^{*}(i n)\right)\right) \leq \eta C \text { if } j>j_{0} .
$$

Therefore,

$$
\left\|f_{k}\right\|_{K, \frac{1}{L n}} \leq \max _{i=0,1, \ldots, j_{0}}\left(\eta C,\left\|P^{i}(D)\left(f_{k}\right)\right\|_{2, K} e^{-\frac{1}{L n} \varphi^{*}(i L n)}\right) .
$$

Since $\left\{f_{k}\right\}_{k \in \mathbb{N}}$ converges to 0 in $\mathcal{E}(\Omega)$ we have $\left\|f_{k}\right\|_{K, \frac{1}{L n}} \leq \eta C$ if $k$ is large enough. The property $\left(\mathrm{P}^{*}\right)$ is satisfied. Indeed, take $l=n, N=1, K=M$ and $S=1$, then

$$
\begin{gathered}
\forall n \forall m \geq n \forall M \in \mathbb{N} \forall x \in X_{n} \\
\|x\|_{n, M} \leq\|x\|_{m, M}+\|x\|_{n, 1},
\end{gathered}
$$

since

$\|x\|_{n, M}=\max \left(\|x\|_{K, \frac{1}{n}}, p_{M}(x)\right)=\left\{\begin{array}{l}\|x\|_{K, \frac{1}{n}} \leq\|x\|_{n, 1} \leq\|x\|_{m, M}+\|x\|_{n, 1} \\ p_{M}(x) \leq\|x\|_{m, M} \leq\|x\|_{m, M}+\|x\|_{n, 1}\end{array}\right.$

To summarize we give the following corollary.

Corollary 2.2.10. The following statements are equivalent:

1. $P$ is hypoelliptic.

2. The topology $\tau_{(\omega), \infty}$ on $\mathcal{E}_{P,(\omega)}(\Omega)$ (respec. $\tau_{\{\omega\}, \infty}$ on $\mathcal{E}_{P,\{\omega\}}(\Omega)$ ) considered in Proposition 2.2.6 (respect in Proposition 2.2.7) coincides with the natural topology of $\mathcal{E}_{P,(\omega)}(\Omega)$ (respec. on $\mathcal{E}_{P,\{\omega\}}(\Omega)$ ).

3. The inclusion $\mathcal{E}_{P, *}(\Omega) \hookrightarrow \mathcal{E}(\Omega)$ is continuous.

4. $\mathcal{E}_{P, *}(\Omega)$ is complet.

Proof. $1 \Rightarrow 2$

Beurling case:

Since $P$ is hypoelliptic, Theorem 2.2.5 implies the completeness of $\mathcal{E}_{P,(\omega)}(\Omega)$ with its natural topology. It is clear that the identity map

$$
\text { Id : }\left(\mathcal{E}_{P,(\omega)}(\Omega), \max \left(\|\cdot\|_{n}, p_{n}\right)\right) \longrightarrow\left(\mathcal{E}_{P,(\omega)}(\Omega),\|\cdot\|_{n}\right)
$$

is continuous and by the Open Mapping Theorem (see [54, Theorem 24.30]) it is a topological homeomorphism. 


\section{Roumieu case:}

The normed space $\mathcal{E}_{P, \omega}^{\frac{1}{n}}(K)$ is closed in the Fréchet space $\mathcal{E}_{P,(\omega)}(\Omega)$ and hence, $\mathcal{E}_{P, \omega}^{\frac{1}{n}}(K)$ is a Banach space endowed with the $\|\cdot\|_{K, \frac{1}{n}}$-topology. Again by the Open Mapping Theorem, this topology is the same that the topology given by the fundamental system of seminorms $\{\max (\|$. $\left.\left.\|_{K, \frac{1}{n}}, p_{m}\right)\right\}_{m \in \mathbb{N}}$.

$$
\begin{aligned}
& 2 \Rightarrow 3 \text { Obvious. } \\
& 3 \Rightarrow 4
\end{aligned}
$$

Since $\mathcal{E}_{P,(\omega)}(\Omega) \hookrightarrow \mathcal{E}_{P,\{\omega\}}(\Omega)$ is continuous, the inclusion map

$$
\mathcal{E}_{P,(\omega)}(\Omega) \hookrightarrow \mathcal{E}(\Omega)
$$

is continuous. Hence

$$
\text { Id }:\left(\mathcal{E}_{P,(\omega)}(\Omega),\|\cdot\|_{n}\right) \longrightarrow\left(\mathcal{E}_{P,(\omega)}(\Omega), \max \left(\|\cdot\|_{n}, p_{n}\right)\right)
$$

is also continuous. Therefore, any Cauchy sequence $\left\{f_{k}\right\}_{k \in \mathbb{N}}$ in $\left(\mathcal{E}_{P,(\omega)}(\Omega),\|\cdot\|_{n}\right)$, is also a Cauchy sequence in $\left(\mathcal{E}_{P,(\omega)}(\Omega), \max \left(\|\cdot\|_{n}, p_{n}\right)\right)$, which is complete by Theorem 2.2.6. The conclusion in the Beurling case easily follows. As a consequence, (Theorem 2.2.5) $P$ is hypoelliptic and $\mathcal{E}_{P,\{\omega\}}(\Omega)$ is also complete.

$4 \Rightarrow 1$ This is the content of Theorem 2.2.5.

\subsection{A Paley-Wiener type theorem and its consequences}

Let $\omega$ be a weight function and $m \geq 1$, it is easy to prove that $\sigma(t):=$ $\omega\left(t^{\frac{1}{m}}\right)$ is also a weight function. Moreover, $\varphi_{\sigma}^{*}(x)=\varphi_{\omega}^{*}(m x)$.

Suppose $P$ is a hypoelliptic polynomial of order $m$. Our aim is to establish a Paley-Wiener type theorem for the generalized non quasi analytic class $\mathcal{E}_{P, *\left(t \frac{1}{m}\right)}(\Omega)$. In order to guarantee the existence of test functions in this space we show that the class of ultradifferentiable functions $\mathcal{E}_{*}(\Omega)$ is always contained in $\mathcal{E}_{P, *\left(t^{\frac{1}{m}}\right)}(\Omega)$ where $m$ is the degree of $P$. As a consequence, $\mathcal{D}_{*}(\Omega)$ is a subset of $\mathcal{E}_{P, *\left(t^{\frac{1}{m}}\right)}(\Omega)$.

In Theorem 2.3.15 we prove that the test space of $\mathcal{E}_{P, *\left(t^{\frac{1}{m}}\right)}(\Omega)$ is nuclear and in Proposition 2.3.16 we give a sufficient condition to guarantee that these spaces of test functions are an algebra. 
Theorem 2.3.1. Let $\Omega \subset \mathbb{R}^{N}$ be an open subset. For a weight function $\omega$ and a polynomial $P$ with degree $m$, the inclusion $\mathcal{E}_{*(t)}(\Omega) \subseteq \mathcal{E}_{P, *\left(t^{\frac{1}{m}}\right)}(\Omega)$ holds and the inclusion map is continuous.

Notation. $\mathcal{E}_{*(t)}(\Omega) \subseteq \mathcal{E}_{P, *\left(t^{\frac{1}{m}}\right)}(\Omega)$ means that both inclusions $\mathcal{E}_{(\omega)}(\Omega) \subseteq$ $\mathcal{E}_{P,(\sigma)}(\Omega)$ and $\mathcal{E}_{\{\omega\}}(\Omega) \subseteq \mathcal{E}_{P,\{\sigma\}}(\Omega)$ hold

We need the following technical lemma.

Lemma 2.3.2. Given a constant $C \geq 1$ and a weight function $\omega$, there exist two constants $A$ and $B$ (depending on $C$ and $\omega$ ) such that for all $j \in \mathbb{N}$ and $\lambda>0$,

$$
C^{j} \exp \left(\lambda \varphi^{*}\left(\frac{j}{\lambda}\right)\right) \leq \exp \left(\lambda \frac{A}{B}\right) \exp \left(\frac{\lambda}{B} \varphi^{*}\left(\frac{B}{\lambda} j\right)\right) .
$$

Proof. From de definition of Young conjugate we get

$$
\exp \left(\lambda \varphi^{*}\left(\frac{j}{\lambda}\right)\right)=\sup _{s \geq 1} s^{j} \exp (-\lambda \omega(s)) \text {. }
$$

Choose $l \in \mathbb{N}$ such that $2^{l} \geq C$. By condition $(\alpha)$, there exist A and B such that

$$
\omega(t)=\omega\left(C \frac{t}{C}\right) \leq \omega\left(2^{l} \frac{t}{C}\right) \leq A+B \omega\left(\frac{t}{C}\right) .
$$

Then,

$$
\begin{gathered}
C^{j} \exp \left(\lambda \varphi^{*}\left(\frac{j}{\lambda}\right)\right) \stackrel{(2.7)}{=} \sup _{s \geq 1}(s C)^{j} \exp (-\lambda \omega(s))=\sup _{t \geq C} t^{j} \exp \left(-\lambda \omega\left(\frac{t}{C}\right)\right) \stackrel{(2.8)}{\leq} \\
\stackrel{(2.8)}{\leq} \sup _{t \geq 1} t^{j} \exp \left(\lambda\left(\frac{A}{B}-\frac{\omega(t)}{B}\right)\right)=\exp \left(\lambda \frac{A}{B}\right) \sup _{t \geq 1} t^{j} \exp \left(-\lambda \frac{1}{B} \omega(t)\right) \stackrel{(2.7)}{=} \\
\stackrel{(2.7)}{=} \exp \left(\lambda \frac{A}{B}\right) \exp \left(\frac{\lambda}{B} \varphi^{*}\left(\frac{B}{\lambda} j\right)\right) .
\end{gathered}
$$

Proof of Theorem 2.3.1: We set $P(z)=\sum_{|\alpha| \leq m} a_{\alpha} z^{\alpha}, P(D)=\sum_{|\alpha| \leq m} a_{\alpha} D^{\alpha}$ and $M:=\max \left\{\left|a_{\alpha}\right|:|\alpha| \leq m\right\}$. Choose F large enough such that for each $j \in \mathbb{N}, M^{j}(m j)^{N} \leq F^{j}$ and take the constants $A$ and $B$ of Lema 2.3.2 such that for each $j \in \mathbb{N}$,

$$
F^{j} \exp \left(\lambda \varphi^{*}\left(\frac{j}{\lambda}\right)\right) \leq \exp \left(\lambda \frac{A}{B}\right) \exp \left(\frac{\lambda}{B} \varphi^{*}\left(\frac{B}{\lambda} j\right)\right) .
$$


To see $\mathcal{E}_{(\omega(t))}(\Omega) \subseteq \mathcal{E}_{P,\left(\omega\left(t^{\frac{1}{m}}\right)\right)}(\Omega)$, take $f \in \mathcal{E}_{(\omega(t))}(\Omega)$ and we fix $K \subset \subset$ $\Omega, \lambda>0$ and $j \geq 1$. We observe that the polynomial of the operator $P^{j}(D)$ is $P^{j}=P \underbrace{\ldots}_{j} P$ and its degree is $m j$. Moreover, there exists $C>0$ such that for each $\alpha \in \mathbb{N}_{0}^{N}$,

$$
\left\|f^{(\alpha)}\right\|_{2, K} \leq C \exp \left(B \lambda \varphi^{*}\left(\frac{|\alpha|}{B \lambda}\right)\right) .
$$

We can choose $C=p_{K, B \lambda}(f)$. Hence,

$$
\begin{aligned}
\left\|P^{j}(D) f\right\|_{2, K} & \leq M^{j} p_{K, B \lambda}(f) \sum_{|\gamma| \leq m j} \exp \left(B \lambda \varphi^{*}\left(\frac{|\gamma|}{B \lambda}\right)\right) \\
& \leq p_{K, B \lambda}(f) M^{j}(m j)^{N} \exp \left(B \lambda \varphi^{*}\left(\frac{m j}{B \lambda}\right)\right) \\
& \leq p_{K, B \lambda}(f) F^{j} \exp \left(B \lambda \varphi^{*}\left(\frac{m j}{B \lambda}\right)\right) \\
& \stackrel{(2.9)}{\leq} p_{K, B \lambda}(f) \exp (\lambda A) \exp \left(\lambda \varphi^{*}\left(\frac{m j}{\lambda}\right)\right)
\end{aligned}
$$

Therefore,

$$
\left\|P^{j}(D) f\right\|_{2, K} \exp \left(-\lambda \varphi^{*}\left(\frac{m j}{\lambda}\right)\right) \leq p_{K, B \lambda}(f) \exp (\lambda A) .
$$

This proves $\mathcal{E}_{(\omega(t))}(\Omega) \subseteq \mathcal{E}_{P,\left(\omega\left(t^{\frac{1}{m}}\right)\right)}(\Omega)$ and that this inclusion is continuous. This settles the Beurling case.

In the Roumieu case, let $f \in \mathcal{E}_{\{\omega(t)\}}(\Omega)$ be given. For each $K \subset \subset$ $\Omega$ there exists $\lambda>0$ such that $f \in \mathcal{E}_{\omega(t)}^{\lambda}(K)$. Proceeding as above $f \in$ $\mathcal{E}_{P, \omega\left(t^{\frac{1}{m}}\right)}^{\frac{\lambda}{B}}(K) \subset \mathcal{E}_{P,\left\{\omega\left(t^{\frac{1}{m}}\right)\right\}}(\Omega)$. Now, from (2.11) we get the continuity of the inclusion

$$
\mathcal{E}_{\omega(t)}^{\lambda}(K) \hookrightarrow \mathcal{E}_{P, \omega\left(t^{\frac{1}{m}}\right)}^{\frac{\lambda}{B}}(K)
$$

and the theorem follows.

Proposition 2.3.3. $\mathcal{E}_{*}(\Omega)$ is a dense subspace of $\mathcal{E}_{P, *\left(t^{\frac{1}{m}}\right)}(\Omega)$.

Proof. First, we suppose $\Omega=\mathbb{R}^{N}$.

Beurling case. Applying Lema 1.0.3 there exist $L \geq 1$ and $y_{0}>0$ such that

$$
\varphi^{*}(y)-y \geq L \varphi^{*}\left(\frac{y}{L}\right)-L \text { for each } y \geq y_{0}
$$


Take $y=\frac{j m}{\lambda}$. The last inequality implies

$$
\frac{\exp \left(-\lambda \varphi^{*}\left(\frac{m j}{\lambda}\right)\right)}{\exp \left(-\lambda L \varphi^{*}\left(\frac{m j}{\lambda L}\right)\right)} \leq \exp (L \lambda-j m) \longrightarrow 0 \text { if } j \rightarrow+\infty
$$

Using Theorem 2.3.1

$$
\mathcal{D}_{(\omega)}\left(\mathbb{R}^{N}\right) \hookrightarrow \mathcal{E}_{(\omega)}\left(\mathbb{R}^{N}\right) \hookrightarrow \mathcal{E}_{P,\left(\omega\left(t^{\frac{1}{m}}\right)\right)}\left(\mathbb{R}^{N}\right)
$$

Let $f \in \mathcal{E}_{P,(\omega)}\left(\mathbb{R}^{N}\right)$ be given. Proceeding as in [22, Lema 3.8] we take a regularizing sequence $\left\{\rho_{n}\right\}_{n \in \mathbb{N}}$ in $\mathcal{D}_{(\omega)}\left(\mathbb{R}^{N}\right)$ and prove that $f * \rho_{n} \in$ $\mathcal{E}_{(\omega)}\left(\mathbb{R}^{N}\right)$. To get this aim we fix a compact subset $K$ of $\mathbb{R}^{N}$ and $\lambda>0$, then

$$
\begin{aligned}
\sup _{\alpha \in \mathbb{N}_{0}^{N}}\left\|\left(f * \rho_{n}\right)^{(\alpha)}\right\|_{2, K} \exp \left(-\lambda \varphi^{*}\left(\frac{|\alpha|}{\lambda}\right)\right)=\sup _{\alpha \in \mathbb{N}_{0}^{N}}\left\|f * \rho_{n}^{(\alpha)}\right\|_{2, K} \exp \left(-\lambda \varphi^{*}\left(\frac{|\alpha|}{\lambda}\right)\right)= \\
\quad=\sup _{\alpha \in \mathbb{N}_{0}^{N}}\left\|\int_{\operatorname{supp}_{n}} f(\cdot-y) \rho_{n}^{(\alpha)}(y) d y\right\|_{2, K} \exp \left(-\lambda \varphi^{*}\left(\frac{|\alpha|}{\lambda}\right)\right) \leq \\
\leq \sup _{\alpha \in \mathbb{N}_{0}^{N}} \sup _{y \in \operatorname{supp} \rho_{n}}\left|\rho_{n}^{(\alpha)}(y)\right| \exp \left(-\lambda \varphi^{*}\left(\frac{|\alpha|}{\lambda}\right)\right)\left\|\int_{\operatorname{supp}_{\rho_{n}}}|f(\cdot-y)| d y\right\|_{2, K}<\infty .
\end{aligned}
$$

Now, it is enough to show that for each compact subset $K$ of $\mathbb{R}^{N}, \lambda>0$ and $p \in \mathbb{N}$

$$
\left\|f-f * \rho_{n}\right\|_{K, \lambda} \longrightarrow 0 \text { if } n \rightarrow \infty .
$$

Given $\eta>0$, using (2.12) and (2.11) there exists $j_{0} \in \mathbb{N}_{0}$ such that

$$
\begin{gathered}
\left\|P^{j}(D)\left(f-f * \rho_{n}\right)\right\|_{2, K} \exp \left(-\lambda \varphi^{*}\left(\frac{m j}{\lambda}\right)\right) \leq \\
\leq\left(\sup _{i \in \mathbb{N}_{0}}\left\|P^{i}(D)\left(f-f * \rho_{n}\right)\right\|_{2, K} \exp \left(-L \lambda \varphi^{*}\left(\frac{m i}{L \lambda}\right)\right)\right) \frac{e^{-\lambda \varphi^{*}\left(\frac{m j}{\lambda}\right)}}{e^{-L \lambda \varphi^{*}\left(\frac{m j}{L \lambda}\right)}} \leq \\
\leq \exp (\lambda A L)\left(\|f\|_{K, L \lambda}+p_{K, L \lambda B}\left(f * \rho_{n}\right)\right) \frac{e^{-\lambda \varphi^{*}\left(\frac{m j}{\lambda}\right)}}{e^{-L \lambda \varphi^{*}\left(\frac{m j}{L \lambda}\right)}}<\eta \text { if } j>j_{0} .
\end{gathered}
$$

As a consequence,

$$
\left\|f-f * \rho_{n}\right\|_{K, \lambda} \leq \max _{i=0,1, \ldots, j_{0}}\left(\eta,\left\|P^{i}(D)\left(f-f * \rho_{n}\right)\right\|_{2, K} e^{-\lambda \varphi^{*}\left(\frac{m i}{\lambda}\right)}\right) .
$$

Hence

$$
\left\|f-f * \rho_{n}\right\|_{K, \lambda} \leq \eta \text { if } n \text { is large enough. }
$$


Roumieu case. Let $f \in \mathcal{E}_{P,\left\{\omega\left(t^{\frac{1}{m}}\right)\right\}}\left(\mathbb{R}^{N}\right)$. We take $\left\{\rho_{n}\right\}_{n \in \mathbb{N}} \in \mathcal{D}_{(\omega)}\left(\mathbb{R}^{N}\right)$ as above and fix a compact subset $K$ of $\mathbb{R}^{N}$. Since $f \in \mathcal{E}_{P,\left\{\omega\left(\frac{1}{m}\right)\right\}}\left(\mathbb{R}^{N}\right)$, there exists $\lambda>0$ such that $f \in \mathcal{E}_{P, \omega}^{\lambda}(K)$. Proceeding as above $f * \rho_{n} \in$ $\mathcal{E}_{P, \omega}^{\lambda}(K)$ and $f * \rho_{n}$ converges to $f$ in $\mathcal{E}_{P, \omega}^{\lambda}(K)$. As a consequence, $f * \rho_{n}$ converges to $f$ in $\mathcal{E}_{P,\left\{\omega\left(\frac{1}{m}\right)\right\}}\left(\mathbb{R}^{N}\right)$.

To finish, we suppose that $\Omega$ is an arbitrary open subset of $\mathbb{R}^{N}$. We fix a compact subset $K$ and $\lambda>0$ and we choose a compact subset $L$ such that $K \subset \stackrel{\circ}{L} \subset L \subset \Omega$. Let $f \in \mathcal{E}_{P,\left(\omega\left(t \frac{1}{m}\right)\right)}(\Omega)$ be given. We define $\widetilde{f}=f$ on $L$ and $\widetilde{f}=0$ in other case. Then $\widetilde{f} \in \mathcal{D}_{(\omega)}^{\prime}\left(\mathbb{R}^{N}\right)$ and according to Proposition 1.0.12 $\rho_{n} * \widetilde{f}$ is an ultradifferentiable function which coincides with $f * \rho_{n}$ on $K$ if $n$ is large enough. Then, given $\varepsilon>0$ there exists $n_{0}$ such that $\left\|f-\rho_{n_{0}} * \widetilde{f}\right\|_{K, \lambda}<\varepsilon$. From this the conclusion follows.

As a consequence of the former results we get:

1. $\mathcal{D}_{*}(\Omega)$ is a dense subset of $\mathcal{E}_{P, *\left(t^{\frac{1}{m}}\right)}(\Omega)$.

2. The functions in $\mathcal{E}_{P, *\left(t^{\frac{1}{m}}\right)}(\Omega)$ with compact support are a dense subset of $\mathcal{E}_{P, *\left(t \frac{1}{m}\right)}(\Omega)$.

Let $K$ be a convex compact subset of $\mathbb{R}^{N}$. Recall that the supporting function of $K$ is the function $H_{K}: \mathbb{R}^{N} \rightarrow \mathbb{R}$ given by $H_{K}(x):=\sup _{y \in K} x \cdot y$.

Lemma 2.3.4. Let $\omega$ a weight function, $P$ a polynomial and $f \in \mathcal{C}^{\infty}\left(\mathbb{R}^{N}\right)$. The following statements hold:

1. If there is $\lambda>0$ satisfying

$$
C:=\left(\int_{\mathbb{R}^{N}}|\widehat{f}(\xi)|^{2} \exp (\lambda \omega(|P(\xi)|)) d \xi\right)^{\frac{1}{2}}<\infty,
$$

then

$$
(*) \sup _{j \in \mathbb{N}_{0}}\left\|P^{j}(D) f\right\|_{2, \mathbb{R}^{\mathbb{N}}} \exp \left(-\frac{\lambda}{2} \varphi^{*}\left(\frac{2 j}{\lambda}\right)\right) \leq \frac{C}{(2 \pi)^{\frac{N}{2}}} .
$$


2. Let $K$ be a compact convex subset of $\mathbb{R}^{N}$ and denote by $m(K)$ the Lebesgue measure of $K$. If $\left(^{*}\right)$ holds and $f$ has compact support contained in $K$, there is a constant $D>0$ (depending on $\lambda$ and $\omega$ ) such that for all $z \in \mathbb{C}^{N}$ the following inequality holds

$$
|\widehat{f}(z)| \leq m(K)^{\frac{1}{2}} \frac{C D}{(2 \pi)^{\frac{N}{2}}} \exp \left(H_{K}(\operatorname{Imz})-\frac{\lambda}{4} \omega(|P(z)|)\right) .
$$

Proof. (1) By Plancherel's Theorem,

$$
\begin{gathered}
\left\|P^{j}(D) f\right\|_{2, \mathbb{R}^{N}}=\frac{1}{(2 \pi)^{\frac{N}{2}}}\left\|P(\xi)^{j} \widehat{f}(\xi)\right\|_{2, \mathbb{R}^{N}} \leq \\
\leq \frac{C}{(2 \pi)^{\frac{N}{2}}} \sup _{|P(\xi)| \neq 0}|P(\xi)|^{j} \exp \left(-\frac{\lambda}{2} \omega(|P(\xi)|)\right) \leq \\
\leq \frac{C}{(2 \pi)^{\frac{N}{2}}} \sup _{|P(\xi)| \neq 0} \exp \left(j \ln |P(\xi)|-\frac{\lambda}{2} \omega(|P(\xi)|)\right) \leq \\
\leq \frac{C}{(2 \pi)^{\frac{N}{2}}} \exp \left(\frac{\lambda}{2} \varphi^{*}\left(\frac{j}{\frac{\lambda}{2}}\right)\right) .
\end{gathered}
$$

(2) Applying Hölder inequality,

$$
\begin{gathered}
\left|\widehat{P^{j}(D) f} f(z)\right| \leq \int_{K}\left|P^{j}(D) f(t) \exp (-i t z)\right| d t \leq \\
\leq m(K)^{\frac{1}{2}} \exp \left(H_{K}(I m z)\right) \frac{C}{(2 \pi)^{\frac{N}{2}}} \exp \left(\frac{\lambda}{2} \varphi^{*}\left(\frac{j}{\frac{\lambda}{2}}\right)\right) .
\end{gathered}
$$

Suppose $|P(z)|>1$, then

$$
|\widehat{f}(z)| \leq m(K)^{\frac{1}{2}} \frac{C}{(2 \pi)^{\frac{N}{2}}} \exp \left(H_{K}(\operatorname{Imz} z)\right) \exp \left(\frac{\lambda}{2} \varphi^{*}\left(\frac{j}{\frac{\lambda}{2}}\right)\right) \frac{1}{|P(z)|^{j}} .
$$

Now, we use condition $\gamma$ of weight function in order to find $t_{0}$ such that

$$
\frac{\ln (t)}{\frac{\lambda}{2}} \leq \frac{1}{2} \omega(t)+\frac{\ln \left(t_{0}\right)}{\frac{\lambda}{2}} \forall t>0 .
$$


Observe that

$$
\begin{aligned}
\sup _{j \in \mathbb{N}_{0}} j \ln |P(z)|-\frac{\lambda}{2} \varphi^{*}\left(\frac{j}{\frac{\lambda}{2}}\right) & =\frac{\lambda}{2} \sup _{j \in \mathbb{N}_{0}}\left(\frac{j+1}{\frac{\lambda}{2}} \ln |P(z)|-\varphi^{*}\left(\frac{j}{\frac{\lambda}{2}}\right)\right)-\ln |P(z)| \\
& \geq \frac{\lambda}{2} \sup _{x \geq 0}\left(x \ln |P(z)|-\varphi^{*}(x)\right)-\ln |P(z)| \\
& =\frac{\lambda}{2}\left(\varphi^{* *}(\ln |P(z)|)-\ln |P(z)|\right. \\
& =\frac{\lambda}{2}(\varphi(\ln |P(z)|))-\ln |P(z)| \\
& =\frac{\lambda}{2} \omega(|P(z)|)-\ln |P(z)| \\
& \geq \frac{\lambda}{4} \omega(|P(z)|)-\ln t_{0} .
\end{aligned}
$$

Taking the infimum

$$
|\widehat{f}(z)| \leq m(K)^{\frac{1}{2}} \frac{C}{(2 \pi)^{\frac{N}{2}}} \exp \left(H_{K}(\operatorname{Imz} z) t_{0} \exp \left(-\frac{\lambda}{4} \omega(|P(z)|)\right) .\right.
$$

In case that $|P(z)| \leq 1$, the inequality (2.14) is also true. To show that, recall that $\omega(|P(z)|)=0$ and $\varphi^{*}(0)=0$ and take $j=0$ in (2.13).

Given $K \subset \subset \Omega$ a compact subset and $\lambda>0$, recall that

$$
\|f\|_{K, \lambda}=\sup _{j \in \mathbb{N}_{0}}\left\|P^{j}(D) f\right\|_{2, K} \exp \left(-\lambda \varphi^{*}\left(\frac{j}{\lambda}\right)\right) .
$$

We use the following notation:

$$
\mathcal{D}_{P, \omega}^{\lambda}(K)=\left\{f \in \mathcal{C}^{\infty}\left(\mathbb{R}^{N}\right): \operatorname{supp} f \subset K \text { and }\|f\|_{K, \lambda}<\infty\right\}
$$

and

$$
\begin{aligned}
& \mathcal{D}_{P,(\omega)}(K)=\operatorname{proj}_{\lambda>0} \mathcal{D}_{P, \omega}^{\lambda}(K), \\
& \mathcal{D}_{P,\{\omega\}}(K)=\operatorname{ind}_{\lambda>0} \mathcal{D}_{P, \omega}^{\lambda}(K) .
\end{aligned}
$$

Definition 2.3.5. Let $\Omega$ be an open subset of $\mathbb{R}^{N}$ and let $\omega$ be a weight function. We define the test spaces of ultradifferentiable functions with respect to the iterates of the operator $P$ as:

$$
\mathcal{D}_{P,(\omega)}(\Omega)=\operatorname{ind}_{K \subset \subset \Omega} \operatorname{proj}_{\lambda>0} \mathcal{D}_{P, \omega}^{\lambda}(K)
$$




$$
\mathcal{D}_{P,\{\omega\}}(\Omega)=\operatorname{ind}_{K \subset \subset \Omega} \operatorname{ind}_{\lambda>0} \mathcal{D}_{P, \omega}^{\lambda}(K)
$$

Given $\lambda>0$, consider the following seminorm

$$
t_{\lambda}(f):=\left(\int_{\mathbb{R}^{N}}|\widehat{f}(\xi)|^{2} \exp (\lambda \omega(|P(\xi)|) d \xi)^{\frac{1}{2}} .\right.
$$

Proposition 2.3.6. Suppose P hypoelliptic. Then, the fundamental systems of seminorms $\left\{\|\cdot\|_{K, \lambda}\right\}_{\lambda>0}$ and $\left\{t_{\lambda}(\cdot)\right\}_{\lambda>0}$ on $\mathcal{D}_{P,(\omega)}(K)$ are equivalent.

Proof. Using Lemma 2.3.4 (1) it is clear that

$$
\|f\|_{K, \lambda} \leq \frac{1}{(2 \pi)^{\frac{N}{2}}} t_{2 \lambda}(f), \forall \lambda>0 \text { and } \forall f \in \mathcal{D}_{P,(\omega)}(K) .
$$

In order to see the other inequality, take $z=\xi \in \mathbb{R}^{N}$ in 2.3.4 (2). Then,

$$
|\widehat{f}(\xi)| \leq m(K)^{\frac{1}{2}} D\|f\|_{K, \frac{\lambda}{2}} \exp \left(-\frac{\lambda}{4} \omega(|P(\xi)|)\right) \text {. }
$$

Then,

$$
|\widehat{f}(\xi)|^{2} \exp \left(\frac{\lambda}{4} \omega(|P(\xi)|)\right) \leq m(K) D^{2}\|f\|_{K, \frac{\lambda}{2}}^{2} \exp \left(-\frac{\lambda}{4} \omega(|P(\xi)|)\right)
$$

Therefore,

$$
t_{\frac{\lambda}{4}}(f) \leq m(K)^{\frac{1}{2}} D\|f\|_{K, \frac{\lambda}{2}}\left(\int_{\mathbb{R}^{N}} \exp \left(-\frac{\lambda}{4} \omega(|P(\xi)|)\right) d \xi\right)^{\frac{1}{2}} .
$$

Now, we only have to check that $\exp \left(-\frac{\lambda}{4} \omega(|P(\xi)|)\right.$ is integrable. Let $F>$ 0 a positive constant. The condition $(\gamma)$ of weight function guarantees

$$
\begin{aligned}
\frac{\lambda}{4} \omega(|P(\xi)|) & \geq(F+1) \ln (|P(\xi)|) \\
& =\ln \left(|P(\xi)||P(\xi)|^{F}\right)
\end{aligned}
$$

if $|\xi|$ is large enough. Condition IIb of [33, Theorem 11.1.3] asserts that there exist $D, d>0$ such that

$$
|P(\xi)| \geq D|\xi|^{d} \text { if }|\xi| \text { is large enough. }
$$


Then, for a suitable $F>0$ we have

$$
\begin{aligned}
\frac{\lambda}{4} \omega(|P(\xi)|) & \geq \ln \left(|P(\xi)||P(\xi)|^{F}\right) \\
& \geq \ln \left(|P(\xi)| D^{F}|\xi|^{d F}\right) \\
& \geq \ln \left(1+|\xi|^{2}\right)^{N}
\end{aligned}
$$

if $|\xi|$ is large enough. As a consequence, there is a constant $C>0$ such that $\exp \left(-\frac{\lambda}{4} \omega(|P(\xi)|) \leq \frac{C}{\left(1+|\xi|^{2}\right)^{N}}\right.$ which is integrable.

Remark 2.3.7. Since $P$ is hypoelliptic, according to condition IIb of [33, Theorem 11.1.3], we have $P \approx \widetilde{P}$ if $|\xi|$ is large enough. So, we can replace $P$ by $\widetilde{P}$ in $t_{\lambda}(f)$, i.e,

$$
t_{\lambda}(f):=\left(\int_{\mathbb{R}^{N}}|\widehat{f}(\xi)|^{2} \exp (\lambda \omega(\widetilde{P}(\xi)) d \xi)^{\frac{1}{2}} .\right.
$$

The following Paley-Wiener type theorem in a classic version is proved in [33, I,Theorem 7.3.1].

Theorem 2.3.8. [Paley-Wiener-Schwartz] Let $K$ be a convex compact subset of $\mathbb{R}^{N}$ with supporting function $H_{K}$. If $u$ is a distribution of order $N$ with support contained in $K$, then

$$
|\widehat{u}(z)| \leq C(1+|z|)^{N} e^{H_{K}(\operatorname{Imz})}, \forall z \in \mathbb{C}^{N} .
$$

Conversely, every entire function in $\mathbb{C}^{N}$ satisfying the last inequality is the FourierLaplace transform of a distribution with support contained in $K$.

If $u$ is a $\mathcal{C}^{\infty}$-function with compact support contained in $\mathrm{K}$ there is for every $\mathrm{Na}$ constant $C_{N}$ such that

$$
|\widehat{u}(z)| \leq C_{N}(1+|z|)^{-N} e^{H_{K}(\operatorname{Im} z)}, \forall z \in \mathbb{C}^{N} .
$$

Conversely, every entire function in $\mathbb{C}^{N}$ satisfying for every $N$ the last inequality is the Fourier-Laplace transform of a $\mathcal{C}^{\infty}$-function with compact support contained in $\mathrm{K}$.

The next Paley-Wiener type theorem for $\mathcal{L}^{2}$ is shown in [59, Theorem 19.3] for one variable. A version for several variables is proved in [62, Theorem 4.9]. Denote $B_{A}:=\left\{x \in \mathbb{R}^{N}:|z| \leq A\right\}$. 
Theorem 2.3.9. Suppose $A$ and $C$ are positive constants and $F$ is an entire function in $\mathbb{C}^{N}$ such that

$$
|F(z)| \leq C e^{A|z|} \forall z \in \mathbb{C}^{N}
$$

and

$$
\int_{\mathbb{R}^{N}}|F(x)|^{2} d x<\infty .
$$

Then, there is a function $f \in \mathcal{L}^{2}\left(B_{A}\right)$ vanishing outside $B_{A}$ such that

$$
F(z)=\int_{B_{A}} f(x) e^{-i x z} d x .
$$

With respect to generalized non quasi analytic classes, this Paley-Wiener type theorem holds:

Theorem 2.3.10. Let $P$ be a hypoelliptic polynomial and $\omega$ a weight function. Then, the Fourier-Laplace transform of a function in $\mathcal{D}_{P,(\omega)}\left(\mathbb{R}^{N}\right)$ verifies

$$
|\widehat{f}(z)| \leq C e^{A|z|} \forall z \in \mathbb{C}^{N}
$$

for some constants $C, A>0$ and moreover, for every $\lambda>0$, the estimate

$$
\left(\int_{\mathbb{R}^{N}}|\widehat{f}(x)|^{2} \exp (\lambda \omega(\mid P(x)) \mid) d x\right)^{\frac{1}{2}}<\infty
$$

is fulfilled. Conversely, every entire function satisfying the above conditions is the Fourier-Laplace transform of a function in $\mathcal{D}_{P,(\omega)}\left(\mathbb{R}^{N}\right)$.

Proof. Let $f \in \mathcal{D}_{P,(\omega)}\left(\mathbb{R}^{N}\right)$ be given and take $A>0$ such that supp $f \subset B_{A}$. By Theorem 2.3.8 there is a constant $C>0$ such that $|\widehat{f}(z)| \leq C e^{A|z|} \forall z \in$ $\mathbb{C}^{N}$. Proposition 2.3.6 gives for each $\lambda>0$ the estimate

$$
\left(\int_{\mathbb{R}^{N}}|\widehat{f}(x)|^{2} \exp (\lambda \omega(\mid P(x)) \mid) d x\right)^{\frac{1}{2}}<\infty .
$$

Conversely, suppose $F$ is an entire function in $\mathbb{C}^{N}$ verifying the above estimates. In particular $\int_{\mathbb{R}^{N}}|F(x)|^{2} d x<\infty$. Thus, Theorem 2.3.9 gives a function $f \in \mathcal{L}^{2}\left(B_{A}\right)$ with supp $f \subset B_{A}$ such that $F(z)=\int_{B_{A}} f(x) e^{-i x z} d x$. Note that for each $\lambda>0$,

$$
\begin{aligned}
& \left(\int_{\mathbb{R}^{N}}|\widehat{f}(x)|^{2} \exp (\lambda \omega(\mid P(x)) \mid) d x\right)^{\frac{1}{2}}= \\
= & \left(\int_{\mathbb{R}^{N}}|F(x)|^{2} \exp (\lambda \omega(\mid P(x)) \mid) d x\right)^{\frac{1}{2}}<\infty .
\end{aligned}
$$


By Proposition 2.3.6, in order to show that $f \in \mathcal{D}_{P,(\omega)}\left(\mathbb{R}^{N}\right)$ we check that we can take $f$ in $\mathcal{C}^{\infty}\left(\mathbb{R}^{N}\right)$.

Fix $\lambda>0$ and write

$$
\widehat{f}(x)=\widehat{f}(x) e^{\frac{\lambda}{2} \omega(|P(x)|)} e^{-\frac{\lambda}{2} \omega(|P(x)|)} .
$$

Proceeding as Lemma 2.3.4, we now that the function $e^{-\frac{\lambda}{2} \omega(|P(x)|)}$ is in $\mathcal{L}^{2}$, therefore Hölder inequality implies $\widehat{f} \in \mathcal{L}^{1}$ and by Inversion Formula (see [59, Theorem 9.14])

$$
f(x)=\frac{1}{(2 \pi)^{N}} \int_{\mathbb{R}^{N}} \widehat{f}(\xi) e^{i x \xi} d \xi \text { a.e. }
$$

In order to see that the function

$$
g(x)=\int_{\mathbb{R}^{N}} \widehat{f}(\xi) e^{i x \xi} d \xi
$$

is in $\mathcal{C}^{\infty}$ it is enough to show that for each $\alpha \in \mathbb{N}_{0}^{N}$, the function $\xi^{\alpha} \widehat{f}(\xi) e^{i x \xi}$ is integrable. Observe

$$
\begin{aligned}
& \left|\xi^{(\alpha)}\right||\widehat{f}(\xi)| \leq\left(\left|\xi_{1}\right|+\cdots+\left|\xi_{N}\right|\right)^{|\alpha|}|\widehat{f}(\xi)| \\
& \leq(\sqrt{N}|\xi|)^{|\alpha|}|\widehat{f}(\xi)| \\
& =\sqrt{N}^{|\alpha|} e^{\ln |\xi||\alpha|}|\widehat{f}(\xi)| .
\end{aligned}
$$

Now, condition IIb of [33, II, Theorem 11.1.3] gives two positive constants $C, d>0$ such that

$$
|P(\xi)| \geq C|\xi|^{d} \text { if }|\xi| \text { is large enough. }
$$

In condition IIb we can assume $C>1$. Then,

$$
\ln |\xi|^{|\alpha|} \leq \ln \left(C^{-\frac{|\alpha|}{d}}|P(\xi)|^{\frac{|\alpha|}{d}}\right) \leq \frac{|\alpha|}{d} \ln (|P(\xi)|) .
$$

Condition $(\gamma)$ of weight function guarantees that

$$
\frac{\omega(|P(\xi)|)}{\ln |P(\xi)|} \geq \frac{|\alpha|}{d} \frac{4}{\lambda} \text { if }|\xi| \text { is large enough. }
$$

Finally, if $|\xi|$ is large enough

$$
\left|\xi^{(\alpha)}\right||\widehat{f}(\xi)| \leq \sqrt{N}^{|\alpha|} e^{\frac{\lambda}{4} \omega(|P(\xi)|)}|\widehat{f}(\xi)|=\sqrt{N}^{|\alpha|}|\widehat{f}(\xi)| e^{\frac{\lambda}{2} \omega(|P(\xi)|)} e^{-\frac{\lambda}{4} \omega(|P(\xi)|),}
$$

which is integrable by Hölder inequality. Then,

$$
\exists g^{(\alpha)}(x)=\int_{\mathbb{R}^{N}} \xi^{\alpha} \widehat{f}(\xi) e^{i x \xi} d \xi
$$


Analogously, one can prove the Roumieu case:

Theorem 2.3.11. Let $P$ be a hypoelliptic polynomial and $\omega$ a weight function. Then, the Fourier-Laplace transform of a function in $\mathcal{D}_{P,\{\omega\}}\left(\mathbb{R}^{N}\right)$ verifies

$$
|\widehat{f}(z)| \leq C e^{A|z|} \forall z \in \mathbb{C}^{N}
$$

for some constants $C, A>0$ and for some $\lambda>0$, the estimate

$$
\left(\int_{\mathbb{R}^{N}}|\widehat{f}(x)|^{2} \exp (\lambda \omega(\mid P(x)) \mid) d x\right)^{\frac{1}{2}}<\infty
$$

is fulfilled. Conversely, every entire function satisfying the above conditions is the Fourier-Laplace transform of a function in $\mathcal{D}_{P,\{\omega\}}\left(\mathbb{R}^{N}\right)$.

Our next aim is to prove that the test spaces

$$
\mathcal{D}_{P,(\omega)}(\Omega)=\operatorname{ind}_{K \subset \subset \Omega} \operatorname{proj}_{\lambda>0} \mathcal{D}_{P, \omega}^{\lambda}(K)
$$

and

$$
\mathcal{D}_{P,\{\omega\}}(\Omega)=\operatorname{ind}_{K \subset \subset \Omega} \operatorname{ind}_{\lambda>0} \mathcal{D}_{P, \omega}^{\lambda}(K)
$$

are nuclear whenever the polynomial $P$ is hypoelliptic.

Given $\lambda>0$, we introduce the following seminorm

$$
s_{\lambda}(f)=\int_{\mathbb{R}^{N}}|\widehat{f}(\xi)| \exp (\lambda \omega(\mid P(\xi)) \mid) d \xi .
$$

Given a compact subset $K$ of $\Omega$ we define

$$
\mathcal{D}_{1, P,(\omega)}(K)=\left\{f \in \mathcal{C}^{\infty}\left(\mathbb{R}^{N}\right): \operatorname{supp} f \subset K \text { and } \forall \lambda>0, s_{\lambda}(f)<\infty\right\}
$$

Since $f \in \mathcal{L}^{1}$, its Fourier transform is bounded. Then,

$$
\begin{aligned}
t_{\lambda}(f) & \leq\|\widehat{f}\|_{\infty}^{\frac{1}{2}}\left(\int_{\mathbb{R}^{N}}|\widehat{f}(\xi)| \exp (\lambda \omega(\mid P(\xi)) \mid) d \xi\right)^{\frac{1}{2}} \\
& =\|\widehat{f}\|_{\infty}^{\frac{1}{2}}\left(s_{\lambda}(f)\right)^{\frac{1}{2}}
\end{aligned}
$$

On the other hand, we write

$$
\widehat{f}(\xi) \exp (\lambda \omega(\mid P(\xi)) \mid)=\widehat{f}(\xi) \exp (2 \lambda \omega(\mid P(\xi)) \mid) \exp (-\lambda \omega(\mid P(\xi)) \mid) .
$$


The Hölder inequality and the fact that $\exp (-\lambda \omega(\mid P(\xi)) \mid)$ is in $\mathcal{L}^{2}$ imply the continuous inclusion

$$
\left(\mathcal{D}_{P,(\omega)}(K), t_{\lambda}\right) \hookrightarrow\left(\mathcal{D}_{1, P,(\omega)}(K), s_{\lambda}\right)
$$

The inequalities above shows that the identity

$$
\left(\mathcal{D}_{1, P,(\omega)}(K), s_{\lambda}\right) \rightarrow\left(\mathcal{D}_{P,(\omega)}(K), t_{\lambda}\right)
$$

holds algebraically.

Proposition 2.3.12. Suppose P hypoelliptic. The identity map

$$
\left(\mathcal{D}_{P,(\omega)}(K), t_{\lambda}\right) \rightarrow\left(\mathcal{D}_{P,(\omega)}(K), s_{\lambda}\right)
$$

is an homeomorphism.

Proof. The metrizable space $\left(\mathcal{D}_{P,(\omega)}(K), t_{\lambda}\right)$ is closed in the Fréchet space $\mathcal{E}_{P,(\omega)}\left(\mathbb{R}^{N}\right)$. Hence, $\left(\mathcal{D}_{P,(\omega)}(K), t_{\lambda}\right)$ is a Fréchet space.

The space $\left(\mathcal{D}_{P,(\omega)}(K), s_{\lambda}\right)$ is also complete. In fact, proceeding as in Theorem 2.3.10, each $f \in \mathcal{D}_{P,(\omega)}(K)$ can be written as

$$
f^{(\alpha)}(x)=\frac{1}{(2 \pi)^{N}} \int_{\mathbb{R}^{N}} \xi^{\alpha} \widehat{f}(\xi) e^{i x \xi} d \xi \text { for all } \alpha \in \mathbb{N}_{0}^{N}
$$

Moreover,

$$
\begin{aligned}
\left|f^{(\alpha)}(x)\right| & \leq \frac{1}{(2 \pi)^{N}} \sup _{\xi \neq 0} \exp (-\lambda \omega(|P(\xi)|)+|\alpha| \ln |\xi|) \int_{\mathbb{R}^{N}}|\widehat{f}(\xi)| e^{\lambda \omega(|P(\xi)|)} d \xi \\
& \leq \frac{1}{(2 \pi)^{N}} \exp \left(\lambda \varphi^{*}\left(\frac{|\alpha|}{\lambda}\right)\right) \int_{\mathbb{R}^{N}}|\widehat{f}(\xi)| e^{\lambda \omega(|P(\xi)|)} d \xi \\
& =\frac{1}{(2 \pi)^{N}} \exp \left(\lambda \varphi^{*}\left(\frac{|\alpha|}{\lambda}\right)\right) s_{\lambda}(f) .
\end{aligned}
$$

As a consequence, the inclusion

$$
\left(\mathcal{D}_{P,(\omega)}(K), s_{\lambda}\right) \hookrightarrow \mathcal{D}(K)
$$

is continuous. Let $\left\{f_{n}\right\}$ a Cauchy sequence in $\mathcal{D}_{P,(\omega)}(K)$. There exists $f_{0} \in \mathcal{D}(K)$ such that $\left\{f_{n}\right\} \rightarrow f$ in $\mathcal{D}(K)$. Then, $\widehat{f} \rightarrow \widehat{f}_{0}$ in $\mathcal{S}\left(\mathbb{R}^{N}\right)$. On the other hand, for each $\lambda>0, h_{n}^{\lambda}:=\widehat{f}_{n} \exp (\lambda \omega(|P|))$ defines a Cauchy 
sequence in $\mathcal{L}^{1}$. Then, we find $h_{\lambda} \in \mathcal{L}^{1}$ such that $h_{n}^{\lambda} \rightarrow h_{\lambda}$ in $\mathcal{L}^{1}$ and moreover a subsequence $h_{n_{k}}^{\lambda}$ which converges to $h_{\lambda}$ a.e in the pointwise convergence. Then, $h_{\lambda}=\widehat{f}_{0} \exp (\lambda \omega(|P|)), f_{0} \in \mathcal{D}_{P,(\omega)}(K)$ and $\left\{f_{n}\right\}$ converges to $f_{0}$ in $\left(\mathcal{D}_{P,(\omega)}(K), s_{\lambda}\right)$.

The conclusion follows by the Closed Graph Theorem.

Definition 2.3.13. A polynomial $P(\xi)=\sum_{|\alpha| \leq m} a_{\alpha} \xi^{\alpha}$ of order $m$ is called elliptic if its principal part $\sum_{|\alpha|=m} a_{\alpha} \xi^{\alpha} \neq 0$ whenever $\xi \neq 0$.

Equivalently, $P$ is elliptic if there is a positive constant $C>0$ such that $|\xi|^{m} \leq C(1+|P(\xi)|)$. According to [33, II, Theorem 11.1.10], every elliptic polynomial is hypoelliptic.

Remark 2.3.14. Recall that the seminorms of $\mathcal{D}_{(\omega)}(K)$ are given by

$$
\int_{\mathbb{R}^{N}}|\widehat{f}(\xi)| \exp (\lambda \omega(|\xi|) d \xi .
$$

If $P$ is elliptic of order $m$, we have $|P(\xi)| \approx|\xi|^{m}$. So, the seminorms of $\mathcal{D}_{(\omega)}(K)$ are recovered by the seminomrs

$$
s_{\lambda}(f)=\int_{\mathbb{R}^{N}}|\widehat{f}(\xi)| \exp (\lambda \sigma(\mid P(\xi)) \mid) d \xi,
$$

if we take the weight $\sigma(t)=\omega\left(t^{\frac{1}{m}}\right)$. In fact, we will prove in the next chapter (Theorem 3.1.7) that the ellipticity of $P$ implies $\mathcal{E}_{P, *\left(t^{\frac{1}{m}}\right)}(\Omega)=\mathcal{E}_{*(t)}(\Omega)$.

Theorem 2.3.15. Let $\Omega$ be an open subset of $\mathbb{R}^{N}$ and $K \subset \subset \Omega$ a compact subset. If $P$ is a hypoelliptic polynomial, then the spaces $\mathcal{D}_{P,(\omega)}(\Omega)$ and $\mathcal{D}_{P,\{\omega\}}(\Omega)$ are nuclear.

Proof. We fix a compact subset $K \subset \subset \Omega$ and prove that $\mathcal{D}_{P,(\omega)}(K)$ is nuclear. Then, the space

$$
\mathcal{D}_{P,(\omega)}(\Omega)=\underset{K \subset \subset \Omega}{\operatorname{ind}} \mathcal{D}_{P,(\omega)}(K)
$$

is also nuclear.

We consider $\mathcal{D}_{P,(\omega)}(K)$ endowed with the seminorms $s_{\lambda}$.

Fix $\lambda>0$ and define

$$
\begin{gathered}
\Delta: \mathbb{R}^{N} \rightarrow\left(\mathcal{D}_{P,(\omega)}(K)^{\prime}, \sigma\left(\mathcal{D}_{P,(\omega)}(K)^{\prime}, \mathcal{D}_{P,(\omega)}(K)\right)\right), \\
\Delta(\xi)[f]:=\widehat{f}(\xi) \exp (2 \lambda \omega(|P(\xi)|)) .
\end{gathered}
$$


Note that Lemma 2.3.4 (2) implies for all $\xi \in \mathbb{R}^{N}$, the inequality

$$
|\Delta(\xi)[f]| \leq D t_{8 \lambda}(f)
$$

for some constant $D>0$. Hence, $\Delta(\xi) \in \mathcal{D}_{P,(\omega)}(K)^{\prime}$ and $\Delta$ is a continuous well defined map. Moreover $\Delta\left(\mathbb{R}^{N}\right) \subseteq V^{\circ}$ where $V$ is the absolutely convex zero neighborhood defined by

$$
V:=\left\{f \in \mathcal{D}_{P,(\omega)}(K) \text { such that } t_{8 \lambda}(f) \leq \frac{1}{D}\right\} .
$$

Now, we consider the following map

$$
\mu: \mathcal{C}\left(V^{\circ}\right) \rightarrow \mathbb{R}, \mu(g):=\int_{\mathbb{R}^{N}} g(\Delta(\xi)) \exp (-\lambda \omega(|P(\xi)|)) d \xi .
$$

If $g: V^{\circ} \rightarrow \mathbb{R}$ is a continuous function on $V^{\circ}$, it is clear that

$$
|\mu(g)| \leq\left(\int_{\mathbb{R}^{N}} \exp (-\lambda \omega(|P(\xi)|)) d \xi\right) \sup _{f \in V^{\circ}}|g(f)| .
$$

This fact implies that $\mu$ is a continuous linear map which is positive, i.e, $\mu(g) \geq 0$ whenever $g \geq 0$. So, $\mu$ defines a measure on $\left(V^{\circ}, \sigma^{*}\right)$ and for each $\lambda>0$,

$$
\begin{aligned}
s_{\lambda}(f) & =\int_{\mathbb{R}^{N}}|\widehat{f}(\xi)| \exp (2 \lambda \omega(|P(\xi)|)) \exp (-\lambda \omega(|P(\xi)|)) d \xi \\
& =\int_{\mathbb{R}^{N}}|\Delta(\xi)[f]| \exp (-\lambda \omega(|P(\xi)|)) d \xi \\
& \leq \int_{V^{\circ}}|y(f)| d \mu(y) .
\end{aligned}
$$

That shows that $\mathcal{D}_{P,(\omega)}(K)$ and $\mathcal{D}_{P,(\omega)}(\Omega)$ are nuclear. Following the arguments of Theorem 2.1.11 one can prove that $\mathcal{D}_{P,\{\omega\}}(\Omega)$ is nuclear.

Proposition 2.3.16. Let $P$ be a hypoelliptic polynomial and $\omega$ a weight function such that $\omega(|P(x+y)|) \leq K+K \omega(|P(x)|)+K \omega(|P(y)|)$ for some constant $K>0$. Then $\mathcal{D}_{P, *}(\Omega)$ is an algebra.

Proof. We consider the seminorms $s_{\lambda}(f)=\int_{\mathbb{R}^{N}}|\widehat{f}(x)| \exp (\lambda \omega(\mid P(x)) \mid) d x$. Note that

$$
\begin{gathered}
\int_{\mathbb{R}^{N}}|\widehat{f g}(x)| \exp (\lambda \omega(\mid P(x)) \mid) d x=\int_{\mathbb{R}^{N}}|\widehat{f} * \widehat{g}(x)| \exp (\lambda \omega(\mid P(x)) \mid) d x \leq \\
\leq \int_{\mathbb{R}^{N}} \int_{\mathbb{R}^{N}}|\widehat{f}(y)||\widehat{g}(x-y)| \exp (\lambda \omega(\mid P(x)) \mid) d y d x .
\end{gathered}
$$


The hypothesis gives a positive constant $C>0$ in such a way

$$
s_{\lambda}(f g) \leq C s_{K \lambda}(f) s_{K \lambda}(g)
$$

Example 2.3.17. Consider the hypoelliptic heat polynomial in two variables, $P(t, x)=i t+x^{2}$, and Gevrey weights $\omega(t)=t^{a}$ for $\left.\left.a \in\right] 0, \frac{1}{2}\right]$, then $\mathcal{D}_{P, *}(\Omega)$ is an algebra.

By Theorem 2.3.1, we have $\mathcal{D}_{t^{2 a}}(\Omega) \subset \mathcal{D}_{P, t^{a}}(\Omega)$ and therefore, $\mathcal{D}_{P, t^{a}}(\Omega)$ is non trivial. For $\omega(t)=t^{\frac{1}{2}}$, easily one can check

$$
|P((x, t)+(y, u))|^{\frac{1}{2}} \leq K+K|P(x, t)|^{\frac{1}{2}}+K|P(y, u)|^{\frac{1}{2}} .
$$

We call $X=P(x+y, t+u), Y=P(x, t)$ and $Z=P(x, t)$. For $0<a<\frac{1}{2}$, we want to see

$$
X^{a} \leq K\left(1+Y^{a}+Z^{a}\right)
$$

for some $K>0$.

By the inequality above, we have $X^{\frac{1}{2}} \leq K\left(1+Y^{\frac{1}{2}}+Z^{\frac{1}{2}}\right)$ for all $X, Y, Z \geq 0$. Observe that $p:=\frac{1}{2 a}>1$. Since on $\mathbb{R}^{N}$ all the norms are equivalent we have $\|\cdot\|_{p} \leq D\|\cdot\|_{1}$ for some $D>0$. Then,

$$
\left(1+\left(Y^{a}\right)^{\frac{1}{2 a}}+\left(Z^{a}\right)^{\frac{1}{2 a}}\right)^{2 a} \leq D\left(1+Y^{a}+Z^{a}\right)
$$

As a consequence,

$$
X \leq K^{2}\left(1+Y^{\frac{1}{2}}+Z^{\frac{1}{2}}\right)^{2} \leq K^{2} D\left(1+Y^{a}+Z^{a}\right)^{\frac{1}{a}}
$$

and then

$$
X^{a} \leq K^{2 a} D^{a}\left(1+Y^{a}+Z^{a}\right)
$$




\section{Chapter 3}

\section{The problem of iterates on non-quasianalytic classes}

The aim of this chapter is to extend to the ultradifferentiable setting the results by Komatsu, Newberger-Zielezny and Métivier mentioned in the introduction. After that, we obtain some results on the problem of iterates on non quasi analytic classes. The main result is Theorem 3.2.3. Under the assumption that the weight function $\omega$ verifies a growth condition introduced by Bonet, Meise and Melikhov, this theorem asserts that the equality $\mathcal{E}_{P, *\left(\frac{1}{m}\right)}(\Omega)=\mathcal{E}_{*(t)}(\Omega)$ holds if and only if $P$ is elliptic.

\subsection{The growth of $\mathcal{E}_{P, \omega}(\Omega)$}

According to a well known result of Hörmander (see [32, Theorem 3.2]), if $P$ is a hypoelliptic polynomial and $Q$ any polynomial, there are constants $h>0$ and $C>0$ such that

$$
|Q(\xi)|^{2} \leq C\left(1+|P(\xi)|^{2}\right)^{h}, \forall \xi \in \mathbb{R}^{N}
$$

Moreover, the smallest $h$ with this property is a rational number.

In case $h \leq 1, Q$ is said to be weaker than $P$. We recall that two hypoelliptic polynomials $P$ and $Q$ are equally strong if $\frac{Q(\xi)}{P(\xi)}$ and $\frac{P(\xi)}{Q(\xi)}$ are bounded at infinity in $\mathbb{R}^{N}$.

Given $P$ and $Q$ hypoelliptic polynomials we show the equivalence between the inequality $|Q(\xi)|^{2} \leq C\left(1+|P(\xi)|^{2}\right)^{h}$ and the inclusion $\mathcal{E}_{P, *(t)}(\Omega) \subseteq$ $\mathcal{E}_{Q, *\left(t \frac{1}{h}\right)}(\Omega)$ for weight functions satisfying the following growth condition 
$B-M-M$ :

There exits a constant $H \geq 1$ such that for all $t \geq 0$

$$
2 \omega(t) \leq \omega(H t)+H
$$

This condition is considered by J.Bonet, R. Meise and S.N.Melikhov in [17] in order to characterize those weight functions $\omega$ such that there exists a sequence $\left\{M_{p}\right\}$ with the property that the class of ultradifferentiable functions in the sense of Braun-Meise-Taylor associated to the weight $\omega$ coincides with the non-quasianalytic class in the sense of Komatsu (see definition 1.0.19 and [38]) defined by the sequence $\left\{M_{p}\right\}$. Gevrey weights verify this condition.

We will prove the following theorems:

Theorem 3.1.1. Let $P$ and $Q$ be hypoelliptic polynomials with $h>0$ and $C>0$ such that $|Q(\xi)|^{2} \leq C\left(1+|P(\xi)|^{2}\right)^{h}, \forall \xi \in \mathbb{R}^{N}$. Let $\Omega \subset \mathbb{R}^{N}$ be an open subset and $\omega$ a weight function, then there exits $m_{0}$ such that if $m \geq m_{0}$

$$
\mathcal{E}_{P, *\left(t^{\frac{1}{m}}\right)}(\Omega) \subseteq \mathcal{E}_{Q, *\left(t \frac{1}{m h}\right)}(\Omega),
$$

and the inclusion map is continuous.

Theorem 3.1.2. Let $\Omega \subset \mathbb{R}^{N}$ be an open subset, $\omega$ a weight function satisfying the condition B-M-M, cf. (3.1). Let $P$ be an hypoelliptic polynomial and let $Q$ be an arbitrary polynomial such that $\mathcal{E}_{P, *(t)}(\Omega) \subseteq \mathcal{E}_{Q, *\left(t \frac{1}{h}\right)}(\Omega)$ for some $h \geq 1$. Then

$$
|Q(\xi)|^{2} \leq C\left(1+|P(\xi)|^{2}\right)^{h}, \forall \xi \in \mathbb{R}^{N}
$$

In the proofs we need two technical lemmata.

Lemma 3.1.3. Let $\omega$ be a weight function, $m \geq 1$ and $\gamma, \mu>0$ such that $\gamma \leq \mu m$. Then for each $k \in \mathbb{N}, i=0,1, \ldots, k$ and $\lambda>0$,

$$
k^{i \gamma} \exp \left(\lambda \varphi^{*}\left(\frac{(k-i) \mu m}{\lambda}\right)\right) \leq \exp (\lambda \omega(k)) \exp \left(\lambda \varphi^{*}\left(\frac{k \mu m}{\lambda}\right)\right) .
$$

As a consequence, since $\omega(t)=o(t)$ there exists $C>0$ (depending on $\omega$ ) such that

$$
k^{i \gamma} \exp \left(\lambda \varphi^{*}\left(\frac{(k-i) \mu m}{\lambda}\right)\right) \leq C \exp (\lambda C k) \exp \left(\lambda \varphi^{*}\left(\frac{k \mu m}{\lambda}\right)\right) .
$$


Proof.

$$
\begin{gathered}
k^{i \gamma} \exp \left(\lambda \varphi^{*}\left(\frac{(k-i) \mu m}{\lambda}\right)\right) \exp \left(-\lambda \varphi^{*}\left(\frac{k \mu m}{\lambda}\right)\right)= \\
=k^{i \gamma} \exp \left(-\lambda\left(\varphi^{*}\left(\frac{k \mu m}{\lambda}\right)-\varphi^{*}\left(\frac{(k-i) \mu m}{\lambda}\right)\right)\right) .
\end{gathered}
$$

Since $\varphi^{*}$ is a convex function, we have $\varphi^{*}(A)-\varphi^{*}(B) \geq \varphi^{*}(A-B)$ if $0 \leq$ $B<A$. Therefore,

$$
\begin{aligned}
& k^{i \gamma} \exp \left(\lambda \varphi^{*}\left(\frac{(k-i) \mu m}{\lambda}\right)\right) \exp \left(-\lambda \varphi^{*}\left(\frac{k \mu m}{\lambda}\right)\right) \leq \\
& k^{i \mu m} \exp \left(-\lambda \varphi^{*}\left(\frac{i \mu m}{\lambda}\right)\right)=\exp \left(\lambda\left(\frac{i \mu m \ln (k)}{\lambda}-\sup _{s \geq 0}\left\{\frac{i \mu m s}{\lambda}-\omega\left(e^{s}\right)\right\}\right)\right) \\
& \leq \exp (\lambda \omega(k))
\end{aligned}
$$

taking $s=\ln (k)$ in the last inequality.

The next lemma is stated in [28, Lemma 1.4] without a proof. We include a proof here for the sake of completeness.

Lemma 3.1.4. Let $h>0, \lambda>0$ be positive constants, then for all $t \geq 1$

$$
\text { (1) } \sup _{j \in \mathbb{N}_{0}} t^{j} \exp \left(-\lambda \varphi^{*}\left(\frac{h j}{\lambda}\right)\right) \leq \exp \left(\lambda \omega\left(t^{\frac{1}{h}}\right)\right)
$$

and

$$
\text { (2) } \sup _{j \in \mathbb{N}_{0}} t^{j} \exp \left(-\lambda \varphi^{*}\left(\frac{h j}{\lambda}\right)\right) \geq \frac{1}{t} \exp \left(\lambda \omega\left(t^{\frac{1}{h}}\right)\right) .
$$

Proof. Proof of (1):

$$
\sup _{j \in \mathbb{N}_{0}} t^{j} \exp \left(-\lambda \varphi^{*}\left(\frac{h j}{\lambda}\right)\right)=\sup _{j \in \mathbb{N}_{0}} t^{j} \exp \left(-\lambda \sup _{s \geq 0}\left\{\frac{h j s}{\lambda}-\omega\left(e^{s}\right)\right\}\right)
$$

We take $s=\frac{\ln (t)}{h} \geq 0$, therefore

$$
\sup _{j \in \mathbb{N}_{0}} t^{j} \exp \left(-\lambda \varphi^{*}\left(\frac{h j}{\lambda}\right)\right) \leq \sup _{j \in \mathbb{N}_{0}} t^{j} t^{-j} \exp \left(\lambda \omega\left(t^{\frac{1}{h}}\right)\right)=\exp \left(\lambda \omega\left(t^{\frac{1}{h}}\right)\right) .
$$


In order to show (2), having in mind $j \leq s<j+1$ :

$$
\begin{aligned}
\sup _{j \in \mathbb{N}_{0}} t^{j} \exp \left(-\lambda \varphi^{*}\left(\frac{h j}{\lambda}\right)\right) & =\sup _{j \in \mathbb{N}_{0}} \exp \left(\lambda\left(\frac{j}{\lambda} \ln (t)-\varphi^{*}\left(\frac{h j}{\lambda}\right)\right)\right) \\
& \geq \sup _{s \geq 0} \exp \left(\lambda\left(\frac{s}{\lambda} \ln (t)-\varphi^{*}\left(\frac{h s}{\lambda}\right)\right)-\ln (t)\right) \\
& =\frac{1}{t} \exp \left(\lambda \varphi^{* *}\left(\frac{\ln (t)}{h}\right)\right) \\
& \left.=\frac{1}{t} \exp \left(\lambda \omega\left(t^{\frac{1}{h}}\right)\right)\right) .
\end{aligned}
$$

Proof of Theorem 3.1.1: Using [32, Theorem 3.2] we can suppose $h=\frac{\mu}{v}$, where $\mu, v \in \mathbb{N}$. Then, for some constant $C>0$,

$$
\left|Q^{v}(\xi)\right|^{2} \leq C\left(1+\left|P^{\mu}(\xi)\right|^{2}\right) \xi \in \mathbb{R}^{N} .
$$

Let $\Omega^{\prime}$ be an open subset relatively compact in $\Omega$. Given $\delta>0$, we set $\Omega_{\delta}^{\prime}:=\left\{x \in \Omega^{\prime}: d\left(x, \partial \Omega^{\prime}\right)>\delta\right\}$ where $\partial \Omega^{\prime}$ is the boundary of $\Omega^{\prime}$. The condition (3.2) and [32, Theorem 4.2] imply that there exist $\gamma>0$ and $C$ (which depends of $P, Q$ and the diameter of $\Omega^{\prime}$ ) such that for each $s \geq 0$ and $t>0$,

$\sup _{0<\tau \leq t} \tau^{\gamma}\left\|Q^{v}(D) f\right\|_{2, \Omega_{s+\tau}^{\prime}} \leq C\left\{\sup _{0<\tau \leq t} \tau^{\gamma}\left\|P^{\mu}(D) f\right\|_{2, \Omega_{s+\tau}^{\prime}}+\|f\|_{2, \Omega_{s}^{\prime}}\right\}, f \in \mathcal{C}^{\infty}(\Omega)$.

Moreover, $\gamma=\frac{\mu}{b}$ where $0<b \leq 1$ is the real number which gives (see [32, Theorem 3.1]) the inequality

$$
|\operatorname{grad} P(\xi)|^{2} \leq C\left(1+|P(\xi)|^{2}\right)^{1-b}
$$

for the hypoelliptic polynomial $P$. Hence,

$$
\left\|Q^{v}(D) f\right\|_{2, \Omega_{s+t}^{\prime}} \leq C\left\{\left\|P^{\mu}(D) f\right\|_{2, \Omega_{s}^{\prime}}+t^{-\gamma}\|f\|_{2, \Omega_{s}^{\prime}}\right\}, f \in \mathcal{C}^{\infty}(\Omega) .
$$

Let $k \in \mathbb{N}, k \geq 1, \delta>0$. Applying repeatedly the last inequality to $s=\left(1-\frac{i}{k}\right) \delta, t=\frac{\delta}{k}$ and $Q^{(k-i) v} f$ for $i=0,1, \ldots, k$ we obtain ( see [57, Theorem 1])

$$
\left\|Q^{k v}(D) f\right\|_{2, \Omega_{2 \delta}^{\prime}} \leq C^{k} \sum_{i=0}^{k}\left(\begin{array}{l}
k \\
i
\end{array}\right)\left(\frac{k}{\delta}\right)^{i \gamma}\left\|P^{(k-i) \mu}(D) f\right\|_{2, \Omega^{\prime}} f \in \mathcal{C}^{\infty}(\Omega) .
$$


For $k=0$ this inequality remains true.

On the other hand, for $i=0,1, \ldots, v-1$ we have

$$
\left|Q^{i}(\xi)\right|^{2} \leq 1+\left|Q^{v}(\xi)\right|^{2}, \xi \in \mathbb{R}^{N}
$$

Again, [32, Theorem 4.2] implies $\forall i=0,1, \ldots, v-1$,

$$
\left\|Q^{i}(D) f\right\|_{2, \Omega_{2 \delta}^{\prime}} \leq C^{\prime}\left\{\left\|Q^{v}(D) f\right\|_{2, \Omega_{\delta}^{\prime}}+\|f\|_{2, \Omega_{\delta}^{\prime}}\right\}, f \in \mathcal{C}^{\infty}(\Omega) .
$$

where $C^{\prime}$ depends of $\delta$.

For $j \in \mathbb{N}_{0}$, we put $j=k v+l, k, l \in \mathbb{N}_{0}, l \leq v-1$. Applying (3.4) to $i=l$ and $Q^{k v} f$,

$$
\left\|Q^{j}(D) f\right\|_{2, \Omega_{2 \delta}^{\prime}} \leq C^{\prime}\left\{\left\|Q^{(k+1) v}(D) f\right\|_{2, \Omega_{\delta}^{\prime}}+\left\|Q^{k v}(D) f\right\|_{2, \Omega_{\delta}^{\prime}}\right\}, f \in \mathcal{C}^{\infty}(\Omega) .
$$

As a consequence, using (3.3) we obtain

$$
\begin{gathered}
\left\|Q^{j}(D) f\right\|_{2, \Omega_{2 \delta}^{\prime}} \leq C^{\prime} C^{k+1} \sum_{i=0}^{k+1}\left(\begin{array}{c}
k+1 \\
i
\end{array}\right)\left(\frac{k+1}{\delta}\right)^{i \gamma}\left\|P^{(k+1-i) \mu}(D) f\right\|_{2, \Omega^{\prime}}+ \\
+C^{\prime} C^{k} \sum_{i=0}^{k}\left(\begin{array}{c}
k \\
i
\end{array}\right)\left(\frac{k}{\delta}\right)^{i \gamma}\left\|P^{(k-i) \mu}(D) f\right\|_{2, \Omega^{\prime}}, f \in \mathcal{C}^{\infty}(\Omega)
\end{gathered}
$$

We set $m_{0}:=\frac{1}{b} \geq 1$. Let $\Omega$ be an open subset of $\mathbb{R}^{N}$ and let $m \geq m_{0}$, $f \in \mathcal{E}_{P,\left(\omega\left(t^{\frac{1}{m}}\right)\right)}(\Omega)$. We fix $K \subset \subset \Omega$ and $\lambda>0$. There exist $\Omega^{\prime}$ and $\delta>0$ such that $K \subset \Omega_{2 \delta}^{\prime} \subset \overline{\Omega^{\prime}} \subset \subset \Omega$. We call $E$ the constant of Lemma 3.1.3 such that for each $k \in \mathbb{N}, i=0,1, \ldots, k$ and $\lambda>0$,

$$
k^{i \gamma} \exp \left(\lambda \varphi^{*}\left(\frac{(k-i) \mu m}{\lambda}\right)\right) \leq \exp (\lambda E k) \exp \left(\lambda \varphi^{*}\left(\frac{k \mu m}{\lambda}\right)\right) .
$$

Denote $F:=2 C\left(\frac{1}{\delta}\right)^{\gamma} e^{\lambda E}$. We can suppose $F \geq 1$ and $\frac{1}{\delta}>1$. We take $A$ and $B$ the constants of Lemma 2.3.2 such that $\forall k \in \mathbb{N}$ and $\forall \lambda>0$,

$$
F^{k} \exp \left(\lambda \varphi^{*}\left(\frac{k}{\lambda}\right)\right) \leq \exp \left(\lambda \frac{A}{B}\right) \exp \left(\frac{\lambda}{B} \varphi^{*}\left(\frac{B}{\lambda} k\right)\right) .
$$

We apply the inequality (3.6) and $f \in \mathcal{E}_{P,\left(\omega\left(t^{\frac{1}{m}}\right)\right)}(\Omega)$ to get

$$
\begin{gathered}
\left\|Q^{j}(D) f\right\|_{2, K} \leq\left\|Q^{j}(D)\right\|_{2, \Omega_{2 \delta}^{\prime}} \leq \\
\leq C^{\prime \prime} C^{k+1} \sum_{i=0}^{k+1}\left(\begin{array}{c}
k+1 \\
i
\end{array}\right)\left(\frac{k+1}{\delta}\right)^{i \gamma} \exp \left(2^{p} B \lambda \varphi^{*}\left(\frac{(k+1-i) \mu m}{2^{p} B \lambda}\right)\right)+
\end{gathered}
$$




$$
+C^{\prime \prime} C^{k} \sum_{i=0}^{k}\left(\begin{array}{l}
k \\
i
\end{array}\right)\left(\frac{k}{\delta}\right)^{i \gamma} \exp \left(B \lambda \varphi^{*}\left(\frac{(k-i) \mu m}{B \lambda}\right)\right)
$$

when $p$ is the first entire after $\mu m$. Therefore,

$$
\begin{gathered}
\left\|Q^{j}(D) f\right\|_{2, K} \leq \\
C^{\prime \prime} C^{k+1} \sum_{i=0}^{k+1}\left(\begin{array}{c}
k+1 \\
i
\end{array}\right)\left(\frac{k+1}{\delta}\right)^{i \gamma} \exp \left(2^{p} B \lambda \varphi^{*}\left(\frac{(k-i) \mu m+p}{2^{p} B \lambda}\right)\right)+ \\
+C^{\prime \prime} C^{k} \sum_{i=0}^{k}\left(\begin{array}{c}
k \\
i
\end{array}\right)\left(\frac{k}{\delta}\right)^{i \gamma} \exp \left(B \lambda \varphi^{*}\left(\frac{(k-i) \mu m}{B \lambda}\right)\right)
\end{gathered}
$$

since $\varphi^{*}$ is increasing. Now, using Lemma 1.0.4 and Lemma 3.1.3 we have

$$
\begin{aligned}
\left\|Q^{j}(D) f\right\|_{2, K} & \leq D\left(2 C\left(\frac{1}{\delta}\right)^{\gamma} e^{B \lambda E}\right)^{k} \exp \left(B \lambda \varphi^{*}\left(\frac{k \mu m}{B \lambda}\right)\right)= \\
& =D F^{k} \exp \left(B \lambda \varphi^{*}\left(\frac{k \mu m}{B \lambda}\right)\right) .
\end{aligned}
$$

In view of lemma 2.3.2,

$$
\begin{gathered}
\left\|Q^{j}(D) f\right\|_{2, K} \leq D \exp \left(B \lambda \frac{A}{B}\right) \exp \left(\lambda \varphi^{*}\left(\frac{k \mu m}{\lambda}\right)\right)= \\
=D \exp (A \lambda) \exp \left(\lambda \varphi^{*}\left(\frac{k h \nu m}{\lambda}\right)\right) \leq D \exp (A \lambda) \exp \left(\lambda \varphi^{*}\left(\frac{j h m}{\lambda}\right)\right) .
\end{gathered}
$$

As a consequence for each $j \in \mathbb{N}$,

$$
\left\|Q^{j}(D) f\right\|_{2, K} \exp \left(-\lambda \varphi^{*}\left(\frac{j h m}{\lambda}\right)\right) \leq D \exp (A \lambda) .
$$

Then,

$$
f \in \mathcal{E}_{Q,\left(\omega\left(t \frac{1}{m h}\right)\right)}(\Omega) .
$$

In order to see the inclusion map

$$
\mathcal{E}_{P,\left(\omega\left(t^{\frac{1}{m}}\right)\right)}(\Omega) \hookrightarrow \mathcal{E}_{Q,\left(\omega\left(t^{\frac{1}{m h}}\right)\right)}(\Omega)
$$

is continuous, let $f \in \mathcal{E}_{P,\left(\omega\left(t^{\frac{1}{m}}\right)\right)}(\Omega)$ and we fix $K \subset \subset \Omega, \lambda>0$. Proceeding as above:

$$
\begin{gathered}
\left\|Q^{j}(D) f\right\|_{2, K} \exp \left(-\lambda \varphi^{*}\left(\frac{j h m}{\lambda}\right)\right) \leq \\
\leq C^{\prime} C^{k+1} \sum_{i=0}^{k+1}\left(\begin{array}{c}
k+1 \\
i
\end{array}\right)\left(\frac{k+1}{\delta}\right)^{i \gamma} \exp \left(-\lambda \varphi^{*}\left(\frac{j h m}{\lambda}\right)\right)\left\|P^{(k+1-i) \mu}(D) f\right\|_{2, \Omega^{\prime}}+
\end{gathered}
$$




$$
+C^{\prime} C^{k} \sum_{i=0}^{k}\left(\begin{array}{l}
k \\
i
\end{array}\right)\left(\frac{k}{\delta}\right)^{i \gamma} \exp \left(-\lambda \varphi^{*}\left(\frac{j h m}{\lambda}\right)\right)\left\|P^{(k-i) \mu}(D) f\right\|_{2, \Omega^{\prime}} .
$$

Note by Lemma 3.1.3, for each $k \in \mathbb{N}, i=0,1, \ldots, k$ and $\lambda>0$,

$$
k^{i \gamma} \exp \left(-\lambda \varphi^{*}\left(\frac{j h m}{\lambda}\right)\right) \leq \exp (\lambda E k) \exp \left(-\lambda \varphi^{*}\left(\frac{(k-i) \mu m}{\lambda}\right)\right) .
$$

So, for each $j \in \mathbb{N}$,

$$
\begin{gathered}
\left\|Q^{j}(D) f\right\|_{2, K} \exp \left(-\lambda \varphi^{*}\left(\frac{j h m}{\lambda}\right)\right) \leq \\
\leq D F^{k} \sup _{l \in \mathbb{N}_{0}}\left\|P^{l}(D) f\right\|_{2, \overline{\Omega^{\prime}}} \exp \left(-\lambda \varphi^{*}\left(\frac{l m}{\lambda}\right)\right) .
\end{gathered}
$$

Hence,

$$
\begin{gathered}
\left\|Q^{j}(D) f\right\|_{2, K} \exp \left(-\frac{\lambda}{B} \varphi^{*}\left(\frac{j h m B}{\lambda}\right)\right) \leq \\
\leq D \exp \left(\lambda \frac{A}{B}\right) \sup _{l \in \mathbb{N}_{0}}\left\|P^{l}(D) f\right\|_{2, \overline{\Omega^{\prime}}} \exp \left(-\lambda \varphi^{*}\left(\frac{l m}{\lambda}\right)\right) .
\end{gathered}
$$

Proceeding as in Theorem 2.3.1, the Roumieu case is analogous.

Proof of Theorem 3.1.2:

Roumieu Case. We fix a compact subset $K_{0} \subset \subset \Omega$. The following inclusions hold:

$$
\mathcal{E}_{P,(\omega(t))}(\Omega) \subseteq \mathcal{E}_{P,\{\omega(t)\}}(\Omega) \subseteq \mathcal{E}_{Q,\left\{\omega\left(t^{\frac{1}{h}}\right)\right\}}(\Omega) \subseteq \underset{n \in \mathbb{N}}{\underset{\operatorname{ind}}{\longrightarrow}} \mathcal{E}_{Q, \omega\left(t^{\frac{1}{h}}\right)}^{\frac{1}{n}}\left(K_{0}\right)
$$

From theorem 2.2.5 we get that $\mathcal{E}_{P,(\omega(t))}(\Omega)$ is a Fréchet space. Now we consider on $\mathcal{E}_{Q, \omega\left(t^{\frac{1}{h}}\right)}^{\frac{1}{n}}\left(K_{0}\right)$ the topology of Theorem 2.2.7, so that $\underset{n \in \mathbb{N}}{\stackrel{\text { ind } \mathcal{E}^{\frac{1}{n}}}{\longrightarrow}\left(\omega\left(t^{\frac{1}{h}}\right)\right.}\left(K_{0}\right)$ is an (LF)-space. By Closed Graph Theorem and Grothendieck's Factorization Theorem (see [54, Theorems 24.31 and 24.33]), there exists $n_{0} \in \mathbb{N}$ such that

$$
\mathcal{E}_{P,(\omega(t))}(\Omega) \subseteq \mathcal{E}_{Q, \omega\left(t \frac{1}{h}\right)}^{\frac{1}{n_{0}}}\left(K_{0}\right)
$$

with continuous inclusion. So, given any seminorm $\max \left(p_{m},\|\cdot\|_{Q, K_{0}, \frac{1}{n_{0}}}\right)$, of $\mathcal{E}_{Q, \omega\left(t \frac{1}{h}\right)}^{\frac{1}{n_{0}}}\left(K_{0}\right)$, there exist $C>0$, a compact $K \subset \subset \Omega, p \in \mathbb{N}_{0}$ and $\lambda>0$ 
such that, for all $f \in \mathcal{E}_{P,(\omega(t))}(\Omega)$,

$$
\begin{gathered}
\sup _{j \in \mathbb{N}_{0}}\left\|Q^{j}(D) f\right\|_{2, K_{0}} \exp \left(-\frac{1}{n_{0}} \varphi^{*}\left(h j n_{0}\right)\right) \leq \max \left(p_{m},\|f\|_{Q, K_{0}, \frac{1}{n_{0}}}\right) \leq \\
\leq C \sup _{j \in \mathbb{N}_{0}}\left\|P^{j}(D) f\right\|_{2, K} \exp \left(-\lambda \varphi^{*}\left(\frac{j}{\lambda}\right)\right) .
\end{gathered}
$$

If $\xi \in \mathbb{R}^{N}$, we denote $f_{\xi}(x)=e^{i<x, \xi>}$ and observe that

$$
Q^{j}(D) e^{i<x, \xi>}=Q(\xi)^{j} e^{i<x, \xi>} \text { and } f_{\xi}^{\alpha}(x)=\xi^{\alpha} i^{\alpha} e^{i<x, \xi>} .
$$

Moreover, $f_{\xi} \in \mathcal{E}_{P,(\omega)}(\Omega)$ since for a compact subset $K \subset \subset \Omega$ and $\lambda>0$

$$
\left\|P^{j}(D) f_{\xi}\right\|_{2, K}=m(K)|P(\xi)|^{j} \leq C \exp \left(\lambda \varphi^{*}\left(\frac{j}{\lambda}\right)\right)
$$

by Lemma 2.3.2.

Applying inequality (3.7) to $f_{\xi}$ we get

$$
\sup _{j \in \mathbb{N}_{0}}|Q(\xi)|^{j} \exp \left(-\frac{1}{n_{0}} \varphi^{*}\left(h j n_{0}\right)\right) \leq C_{2} \sup _{j \in \mathbb{N}_{0}}|P(\xi)|^{j} \exp \left(-\lambda \varphi^{*}\left(\frac{j}{\lambda}\right)\right) .
$$

For $|Q(\xi)|$ and $|P(\xi)|$ greater or equal than 1 we obtain from (3.8) and Lemma 3.1.4,

$$
\exp \left(\frac{1}{2 n_{0}} \omega\left(|Q(\xi)|^{\frac{1}{h}}\right)\right) \leq C_{2} \exp (\lambda \omega(|P(\xi)|)) .
$$

Hence,

$$
\omega\left(|Q(\xi)|^{\frac{1}{h}}\right) \leq C_{3}+C_{4} \omega(|P(\xi)|) \leq C_{5} \omega(|P(\xi)|)
$$

whenever $|P(\xi)|,|Q(\xi)| \geq 1$. On the other hand, condition B-M-M implies that for each $k \in \mathbb{N}$ there exists $H_{k}$ such that $2^{k-1} \omega(t) \leq \omega\left(H_{k} t\right)$ whenever $t \geq 1$. Then,

$$
\omega\left(|Q(\xi)|^{\frac{1}{h}}\right) \leq \omega\left(C_{6}|P(\xi)|\right) \text { whenever }|P(\xi)|,|Q(\xi)| \geq 1 .
$$

Having in mind $\omega$ vanishes on $[0,1]$ and it is increasing and $|P|$ tends to $+\infty$ if $|\xi|$ tends to $+\infty$ we finally conclude that there is $C_{7}>0$ such that,

$$
|Q(\xi)|^{\frac{1}{h}} \leq C_{7}|P(\xi)| \text { for every } \xi \in \mathbb{R}^{N}
$$

The Beurling case is easier because $\mathcal{E}_{P,(\omega)}(\Omega)$ is a Fréchet space. 
Corollary 3.1.5. Let $\omega$ be a weight function given. Let $P$ and $Q$ be hypoelliptic polynomials and let $\Omega$ be an open subset of $\mathbb{R}^{N}$.

1. If $P$ and $Q$ are equally strong then there is $m_{0}$ such that $m \geq m_{0}$ implies $\mathcal{E}_{P, *\left(t^{\frac{1}{m}}\right)}(\Omega)=\mathcal{E}_{Q, *\left(t^{\frac{1}{m}}\right)}(\Omega)$.

2. The converse is true under the additional assumption that $\omega$ satisfies condition $B-M-M$.

Proof. (1) There are constants $C, D>0$ such that $|Q(\xi)|^{2} \leq C\left(1+|P(\xi)|^{2}\right)$ and $|P(\xi)|^{2} \leq C\left(1+|Q(\xi)|^{2}\right), \forall \xi \in \mathbb{R}^{N}$. By Theorem 3.1.1 there are $m_{1}$ and $m_{2}$ such that

$$
\mathcal{E}_{P, *\left(t^{\frac{1}{m}}\right)}(\Omega) \subseteq \mathcal{E}_{Q, *\left(t^{\frac{1}{m}}\right)}(\Omega) \text { if } m \geq m_{1}
$$

and

$$
\mathcal{E}_{Q, *\left(t^{\frac{1}{m}}\right)}(\Omega) \subseteq \mathcal{E}_{P, *\left(t^{\frac{1}{m}}\right)}(\Omega) \text { if } m \geq m_{2} .
$$

Take $m_{0}=\max \left(m_{1}, m_{2}\right)$.

(2) We apply Theorem 3.1.2.

Next we show that for any elliptic polynomial $P$ of degree $m$, the classes $\mathcal{E}_{P, *\left(t^{\frac{1}{m}}\right)}(\Omega)$ and $\mathcal{E}_{, *(t)}(\Omega)$ are the same as sets and as topological vector spaces. This is an extension of a result of Komatsu (see [37]). The converse in the Gevrey setting is due to Métivier [55]. We need the following Lemma due to Komatsu (see [37, Lemma 3]).

Lemma 3.1.6. Let $\Omega$ be an open subset of $\mathbb{R}^{N}$. Suppose that $P$ is an elliptic operator of order $m$ and let $\rho_{0}>0$ be given. Then, there exists a constant $C>0$, which only depends on $N, \rho_{0}$ and $P$, such that for each $f \in \mathcal{C}^{\infty}(\Omega)$ and for each $\alpha \in \mathbb{N}_{0}{ }^{N}$ verifying $|\alpha| \leq m$,

$$
\left\|f^{(\alpha)}\right\|_{2, \Omega_{\rho+\sigma}} \leq C\|P(D) f\|_{2, \Omega_{\sigma}}{ }^{\frac{|\alpha|}{m}}\|f\|_{2, \Omega_{\sigma}}{ }^{1-\frac{|\alpha|}{m}}+C \frac{1}{\rho^{|\alpha|}}\|f\|_{2, \Omega_{\sigma}}
$$

for every $0<\rho \leq \rho_{0}$ and $\sigma>0$.

Theorem 3.1.7. Let $\omega$ be a weight function and let $\Omega$ be an open subset of $\mathbb{R}^{N}$. For any elliptic polynomial $P$ of degree $m$ we have

$$
\mathcal{E}_{P, *\left(t^{\frac{1}{m}}\right)}(\Omega) \subseteq \mathcal{E}_{*(t)}(\Omega)
$$

and the inclusion map is continuous. 
Proof. Let $\Omega^{\prime}$ be an open subset relatively compact in $\Omega$. We first estimate the derivatives $f^{(\alpha)}$ of order $|\alpha|=k m, k \in \mathbb{N}_{0}$. We write $\alpha=p+q$ with $|p|=m$ and $|q|=(k-1) m$. Using Lemma 3.1.6 with $\rho=\frac{\delta}{k}$ and $\sigma=$ $\delta\left(1-\frac{1}{k}\right), \delta$ small enough, we get a constant $C>0$ such that $\forall f \in \mathcal{C}^{\infty}\left(\Omega^{\prime}\right)$,

$\left\|f^{(\alpha)}\right\|_{2, \Omega_{\delta}^{\prime}}=\left\|\left(f^{(q)}\right)^{(p)}\right\|_{2, \Omega_{\delta}^{\prime}} \leq C\left\{\left\|P(D) f^{(q)}\right\|_{2, \Omega_{\delta\left(1-\frac{1}{k}\right)}^{\prime}}+\left(\frac{k}{\delta}\right)^{m}\left\|f^{(q)}\right\|_{2, \Omega_{\delta\left(1-\frac{1}{k}\right)}^{\prime}}\right\}$.

Applying this Lemma $k$ times as is (3.3) we obtain

$$
\left\|f^{(\alpha)}\right\|_{2, \Omega_{\delta}^{\prime}} \leq C^{k} \sum_{i=0}^{k}\left(\begin{array}{l}
k \\
i
\end{array}\right)\left(\frac{k}{\delta}\right)^{i m}\left\|P^{(k-i)}(D) f\right\|_{2, \Omega^{\prime}}
$$

whenever $|\alpha|=k m$. In the case that $\alpha \in \mathbb{N}_{0}{ }^{N}$ is an arbitrary multi-index we write $\alpha=\beta+\gamma$, where $|\beta|=k m$ and $|\gamma|<m$.

We observe that $x^{a} y^{1-a} \leq x+y$ holds if $x, y \geq 0$ and $0<a<1$. Therefore,

$$
\|P(D) f\|_{2, \Omega_{\delta}^{\prime}} \frac{|\gamma|}{m}\|f\|_{2, \Omega_{\delta}^{\prime}}{ }^{1-\frac{|\gamma|}{m}} \leq\|P(D) f\|_{2, \Omega_{\delta}^{\prime}}+\|f\|_{2, \Omega_{\delta}^{\prime}} .
$$

Hence it follows from Lemma 3.1.6 that there is a constant $C^{\prime}$ such that

$$
\left\|f^{(\gamma)}\right\|_{2, \Omega_{2 \delta}^{\prime}} \leq C^{\prime}\left(\|P(D) f\|_{2, \Omega_{\delta}^{\prime}}+\|f\|_{2, \Omega_{\delta}^{\prime}}\right) \forall f \in \mathcal{C}^{\infty}\left(\Omega_{\delta}^{\prime}\right) \text { y } \forall|\gamma|<m
$$

$C^{\prime}$ dependes of $\delta$. We apply (3.10) to $f^{(\beta)}$ to obtain

$$
\left\|f^{(\alpha)}\right\|_{2, \Omega_{2 \delta}^{\prime}} \leq C^{\prime}\left(\left\|P(D) f^{(\beta)}\right\|_{2, \Omega_{\delta}^{\prime}}+\left\|f^{(\beta)}\right\|_{2, \Omega_{\delta}^{\prime}}\right) .
$$

Now the inequality (3.9) implies

$$
\begin{gathered}
\left\|P(D) f^{(\beta)}\right\|_{2, \Omega_{\delta}^{\prime}}=\left\|(P(D) f)^{(\beta)}\right\|_{2, \Omega_{\delta}^{\prime}} \leq C^{k} \sum_{i=0}^{k}\left(\begin{array}{c}
k \\
i
\end{array}\right)\left(\frac{k}{\delta}\right)^{i m}\left\|P^{(k+1-i)}(D) f\right\|_{2, \Omega^{\prime}} \leq \\
\leq C^{k+1} \sum_{i=0}^{k+1}\left(\begin{array}{c}
k+1 \\
i
\end{array}\right)\left(\frac{k+1}{\delta}\right)^{i m}\left\|P^{(k+1-i)}(D) f\right\|_{2, \Omega^{\prime}} .
\end{gathered}
$$

From this inequality, (3.11) and (3.9) we conclude

$$
\begin{aligned}
\left\|f^{(\alpha)}\right\|_{2, \Omega_{2 \delta}^{\prime}} \leq & C^{\prime} C^{k+1} \sum_{i=0}^{k+1}\left(\begin{array}{c}
k+1 \\
i
\end{array}\right)\left(\frac{k+1}{\delta}\right)^{i m}\left\|P^{(k+1-i)}(D) f\right\|_{2, \Omega^{\prime}}+ \\
& +C^{\prime} C^{k} \sum_{i=0}^{k}\left(\begin{array}{c}
k \\
i
\end{array}\right)\left(\frac{k}{\delta}\right)^{i m}\left\|P^{(k-i)}(D) f\right\|_{2, \Omega^{\prime}} .
\end{aligned}
$$


As $\gamma \leq \mu m$, we can use Lemma 3.1.4, proceeding as in Theorem 3.1.1 with $h=\frac{\mu}{v}=\frac{1}{m}$ and $\gamma=\frac{\mu}{b}=m$, to conclude that

$$
\mathcal{E}_{P, *\left(t^{\frac{1}{m}}\right)}(\Omega) \subseteq \mathcal{E}_{*(t)}(\Omega)
$$

with continuous inclusion.

Corollary 3.1.8. Let $\omega$ be a weight function and let $\Omega$ be an open subset of $\mathbb{R}^{N}$ and $P$ an elliptic polynomial. Then the equality $\mathcal{E}_{P, *\left(t^{\frac{1}{m}}\right)}(\Omega)=\mathcal{E}_{*(t)}(\Omega)$ holds.

Proof. It is a consequence of Theorems 2.3.1 and 3.1.7.

Corollary 3.1.9. Let $\omega$ be a weight function and let $\Omega$ be an open subset of $\mathbb{R}^{N}$. If $P$ is a hypoelliptic polynomial, then there exists $m_{0}$ such that $m \geq m_{0}$ implies $\mathcal{E}_{P, *\left(t^{\frac{1}{m}}\right)}(\Omega) \subseteq \mathcal{E}_{*\left(t \frac{1}{m h}\right)}(\Omega)$ with continuous inclusion.

Proof. Condition IIb of [33, Theorem 11.1.3] gives two positive constants $C, h>0$ such that $|\xi| \leq C(1+|P(\xi)|)^{h}$. Consider the elliptic polynomial $Q(\xi)=|\xi|^{2}=\xi_{1}^{2}+\ldots+\xi_{N}^{2}$ of degree 2 . Then, $|Q(\xi)|^{2} \leq C^{\prime}\left(1+|P(\xi)|^{2}\right)^{2 h}$ for an other positive constant $C^{\prime}>0$. Theorem 3.1.1 implies the existence of $m_{0}$ such that $\mathcal{E}_{P, *\left(t^{\frac{1}{m}}\right)}(\Omega) \subseteq \mathcal{E}_{Q, *\left(t \frac{1}{m 2 h}\right)}(\Omega)$ if $m \geq m_{0}$. By Theorem 3.1.7, $\mathcal{E}_{Q, *\left(\frac{1}{m 2 h}\right)}(\Omega)=\mathcal{E}_{*\left(t^{\frac{1}{m h}}\right)}(\Omega)$

\subsection{Results on the problem of iterates}

The problem of iterates consists in characterizing the functions in a given class of functions in terms of the behavior of the iterates of a fixed differential operator.

Let $\omega$ be a weight function and let $P$ be a polynomial of order $m$. In this section we want to characterize when $\mathcal{E}_{P, *\left(t^{\frac{1}{m}}\right)}(\Omega)=\mathcal{E}_{*(t)}(\Omega)$ in terms of the polynomial $P$. First, we show that the coincidence between $\mathcal{E}_{P, *\left(t^{\frac{1}{m}}\right)}(\Omega)$ and $\mathcal{E}_{*(t)}(\Omega)$ implies the hypoellipticity of $P$. For weight functions verifying the growth condition B-M-M, we will prove that this equality holds if and only if the polynomial $P$ is elliptic. The assumption that $\omega$ verifies the property B-B-M is needed in order to achieve this result. In fact, in Corollary 3.2.2 we consider the weight function $\omega(t)=\log ^{\beta}(1+t), \beta>1$, which does not have this property and see that the equality $\mathcal{E}_{P, *\left(t^{\frac{1}{m}}\right)}(\Omega)=$ $\mathcal{E}_{*(t)}(\Omega)$ holds if and only if $P$ is hypoelliptic. 
Lemma 3.2.1. If $\mathcal{E}_{P, *\left(t^{\frac{1}{m}}\right)}(\Omega)=\mathcal{E}_{*(t)}(\Omega)$ algebraically, then $P$ is hypoelliptic and the previous equality also holds in the topological sense.

Proof. We will present the proof for $\Omega=\mathbb{R}^{N}$. Otherwise, we can proceed as in Proposition 2.3.3.

Beurling case:

We put $\sigma(t)=\omega\left(t^{\frac{1}{m}}\right)$. Since

$$
\left\{f \in \mathcal{C}^{\infty}\left(\mathbb{R}^{N}\right): P(D) f=0\right\} \subset \mathcal{E}_{P,(\sigma)}\left(\mathbb{R}^{N}\right),
$$

our hypothesis implies that

$$
\left\{f \in \mathcal{C}^{\infty}\left(\mathbb{R}^{N}\right): P(D) f=0\right\}=\left\{f \in \mathcal{E}_{(\omega)}\left(\mathbb{R}^{N}\right): P(D) f=0\right\} .
$$

We fix a compact subset $K \subset \mathbb{R}^{N}$ and $\lambda>0$. From the Open Mapping Theorem we deduce that there are a constant $C>0, m \in \mathbb{N}$ and a compact set $Q$ such that

$$
p_{K, \lambda}(f) \leq C \sup _{|\alpha| \leq m} \sup _{x \in Q}\left|f^{(\alpha)}(x)\right|
$$

whenever $f \in \mathcal{C}^{\infty}\left(\mathbb{R}^{N}\right)$ and $P(D) f=0$. We now assume that $P$ is not hypoelliptic. Then we can apply Theorem 2.1.4 and Proposition 2.1.7 to find a function $f \in \mathcal{C}^{m}\left(\mathbb{R}^{N}\right) \backslash \mathcal{C}^{\infty}\left(\mathbb{R}^{N}\right)$ such that $P(D) f=0$. We take $\left\{\rho_{n}\right\}$ a regularizing sequence. Then $\left\{f * \rho_{n}\right\}$ is a Cauchy sequence in $\mathcal{C}^{m}\left(\mathbb{R}^{N}\right)$ and from inequality (3.12) we conclude that also $\left\{f * \rho_{n}\right\}$ is a Cauchy sequence in $\mathcal{E}_{(\omega)}\left(\mathbb{R}^{N}\right)$. This is a contradiction.

\section{Roumieu case:}

We fix a compact subset $K \subset \mathbb{R}^{N}$. Now, our hypothesis implies that the inclusion

$$
\left\{f \in \mathcal{C}^{\infty}\left(\mathbb{R}^{N}\right): P(D) f=0\right\} \subseteq \operatorname{ind}_{n} \mathcal{E}_{\omega}^{\frac{1}{n}}(K)
$$

holds. Moreover, the restriction map has closed graph. By Closed Graph Theorem and Grothendieck's Factorization Theorem there exists $n_{0} \in \mathbb{N}$ such that the map $T:\left\{f \in \mathcal{C}^{\infty}\left(\mathbb{R}^{N}\right): P(D) f=0\right\} \rightarrow \mathcal{E}_{\omega}^{\frac{1}{n_{0}}}(K)$ given by $T(f)=f \mid K$ is well defined and continuous. This gives an inequality similar to (3.12) and we can conclude as above. 
Corollary 3.2.2. Let $\Omega$ be an open subset of $\mathbb{R}^{N}$. Let $\omega(t)=\log ^{\beta}(1+t)$, $\beta>1$, be given and $P$ a polynomial. Then $\mathcal{E}_{P, *}(\Omega)=\mathcal{E}_{*}(\Omega)$ holds algebraically if, and only if, $P$ is hypoelliptic. In this case, the equality $\mathcal{E}_{P, *}(\Omega)=\mathcal{E}_{*}(\Omega)$ is also topological.

Proof. If $m \geq 1$, observe that the weight $\sigma(t)=\omega\left(t^{\frac{1}{m}}\right)$ is equivalent to the weight $\omega(t)=\log ^{\beta}(1+t)$. Therefore, in view of Remark 1.0.8, the equalities $\mathcal{E}_{\omega}(\Omega)=\mathcal{E}_{\sigma}(\Omega)$ and $\mathcal{E}_{P, \omega}(\Omega)=\mathcal{E}_{P, \sigma}(\Omega)$ hold in the Beurling and Roumieu cases.

Suppose $\mathcal{E}_{P, *}(\Omega)=\mathcal{E}_{*}(\Omega)$, then $P$ is hypoelliptic by Lemma 3.2.1. Conversely, if $P$ is hypoelliptic, Corollary 3.1.9 asserts the existence of $m_{0}$ large enough and $h>0$ such that $\mathcal{E}_{P, *\left(t^{\frac{1}{m_{0}}}\right)}(\Omega) \subseteq \mathcal{E}_{*\left(t^{\frac{1}{m_{0}}}\right)}(\Omega)$ with continuous inclusion, that is, $\mathcal{E}_{P, *}(\Omega) \subseteq \mathcal{E}_{*}(\Omega)$ with continuous inclusion. The other inclusion is a consequence of Theorem 2.3.1.

For weight functions satisfying the growth condition B-M-M, we can proceed as in Theorem 3.1.2 and obtain the following result:

Theorem 3.2.3. Let $\omega$ be a weight function verifying condition B-M-M. Suppose $P$ is a polynomial which degree is $m$. Then $\mathcal{E}_{P, *\left(t^{\frac{1}{m}}\right)}(\Omega)=\mathcal{E}_{*(t)}(\Omega)$ holds algebraically if and only if $P$ is elliptic. In this case the equality $\mathcal{E}_{P, *\left(t \frac{1}{m}\right)}(\Omega)=$ $\mathcal{E}_{*(t)}(\Omega)$ is also topological.

Proof. If $P$ is elliptic then $\mathcal{E}_{P, *\left(t \frac{1}{m}\right)}(\Omega)=\mathcal{E}_{*(t)}(\Omega)$ by theorem 3.1.7. In order to show the converse, we apply once again Theorem 3.1.7 to the elliptic polynomial $Q(\xi)=\xi_{1}^{2}+\ldots+\xi_{N}^{2}$ of order 2 to get

$$
\mathcal{E}_{P, *\left(t^{\frac{1}{m}}\right)}(\Omega)=\mathcal{E}_{*(t)}(\Omega)=\mathcal{E}_{\tilde{\xi}_{1}^{2}+\ldots+\tilde{\xi}_{N}^{2}, *\left(t^{\frac{1}{2}}\right)}(\Omega) .
$$

Moreover, according to Lemma 3.2.1, $P$ is hypoelliptic and we can proceed as in Theorem 3.1.2 to deduce

$$
\left(\xi_{1}^{2}+\ldots+\xi_{N}^{2}\right)^{\frac{1}{2}} \leq C|P(\xi)|^{\frac{1}{m}} \text { if }|\xi| \text { is large enough. }
$$

That is, for some constant $C>0$,

$$
\left(\xi_{1}^{2}+\ldots+\xi_{N}^{2}\right)^{m} \leq C\left(1+|P(\xi)|^{2}\right) .
$$





\section{Chapter 4}

\section{Fréchet spaces invariant under differentiation}

M. Langenbruch and J. Voigt proved in [50] that a Fréchet space of distributions which is stable under differential operators is continuously included in $\mathcal{C}^{\infty}$. They also showed that to guarantee this continuous inclusion it is enough to assume that the Fréchet space is stable under a single hypoelliptic differential operator $P(D)$ and that this property in fact characterizes the hypoellipticity of the operator. Our aim in this chapter is to provide extensions of these results to the ultradifferentiable setting and show their connection with the problem of iterates of differential operators.

Observe that the class of ultradistributions of Roumieu type

$E:=\mathcal{D}_{\{\omega\}}^{\prime}\left(\mathbb{R}^{N}\right)$ is a Fréchet space invariant under differential operators which is not included in $\mathcal{C}^{\infty}\left(\mathbb{R}^{N}\right)$. Nevertheless, a result similar to that of Langenbruch and Voigt can be obtained after imposing the extra assumption that $E$ is stable under suitable differential operators of infinite order, available in any non quasianalytic class other than $\mathcal{C}^{\infty}\left(\mathbb{R}^{N}\right)$. These operators are called ultradifferentiable operators. After some preliminaries on ultradifferential operators we extend in the second section the result of Langenbruch and Voigt using strongly $(\omega)$-hypoelliptic ultradifferential operators. The main result is Theorem 4.2.1.

Each hypoelliptic linear partial differential operator with constant coefficients is also Gevrey hypoelliptic for some Gevrey class depending on the operator, hence it makes sense to study whether Fréchet spaces of distributions invariant under a single hypoelliptic operator and satisfying some extra assumptions should be contained not only in the space of all smooth functions but in a smaller class of ultradifferentiable func- 
tions. This question is related to the problem of iterates. So, in the last section of this chapter we address the question whether a Fréchet space $E \subset \mathcal{D}_{(\omega)}^{\prime}\left(\mathbb{R}^{N}\right)$ which is invariant under the action of a single one elliptic operator $P(D)$ that satisfies some extra assumptions on equicontinuity is necessarily contained in $\mathcal{E}_{(\omega)}\left(\mathbb{R}^{N}\right)$. The cases that $P(D)$ is hypoelliptic or semielliptic are also considered. As a consequence we obtain several results related to the problem of iterates for non-quasianalytic classes. Main results in this section are Theorem 4.3.4 and Corollary 4.3.9.

\subsection{Preliminaries: ultradifferential operators}

We introduce now the ultradifferential operators with constant coefficients. We follow [21].

Lemma 4.1.1. Let $G \in \mathcal{H}\left(\mathbb{C}^{N}\right)$ be an entire function such that $\log |G(z)|=$ $\mathcal{O}(\omega(|z|))$ as $|z|$ tends to infinity. Then, there is a constant $k>0$ such that

$$
\left|G^{(\alpha)}(0)\right| \leq \alpha ! e^{k} e^{-k \varphi^{*}\left(\frac{|\alpha|}{k}\right)}, \quad \forall \alpha \in \mathbb{N}_{0}^{N}
$$

Definition 4.1.2. Let $G \in \mathcal{H}\left(\mathbb{C}^{N}\right)$ be an entire function such that $\log |G(z)|=$ $\mathcal{O}(\omega(|z|))$ as $|z|$ tends to infinity. Then, the previous Lemma allows to show that

$$
T_{G}(\varphi):=\sum_{\alpha \in \mathbb{N}_{0}^{N}}(-i)^{|\alpha|} \frac{G^{(\alpha)}(0)}{\alpha !} \varphi^{(\alpha)}(0)
$$

defines an ultradistribution $T_{G} \in \mathcal{E}_{(\omega)}^{\prime}\left(\mathbb{R}^{N}\right)$ whose support reduces to $\{0\}$. The convolution operator

$$
G(D): \mathcal{D}_{(\omega)}^{\prime}\left(\mathbb{R}^{N}\right) \rightarrow \mathcal{D}_{(\omega)}^{\prime}\left(\mathbb{R}^{N}\right), \quad G(D) v:=T_{G} * v
$$

is called an ultradifferential operator of class $(\omega)$.

If $f \in \mathcal{E}_{(\omega)}\left(\mathbb{R}^{N}\right)$, then $G(D) f \in \mathcal{E}_{(\omega)}\left(\mathbb{R}^{N}\right) . G(D)$ has the important property that its restriction to $\mathcal{E}_{(\omega)}\left(\mathbb{R}^{N}\right)$ can be interpreted as a differential operator of infinite order

$$
G(D): \mathcal{E}_{(\omega)}\left(\mathbb{R}^{N}\right) \rightarrow \mathcal{E}_{(\omega)}\left(\mathbb{R}^{N}\right)
$$

More precisely, for every $f \in \mathcal{E}_{(\omega)}\left(\mathbb{R}^{N}\right)$ one has,

$$
G(D) f(x)=\sum_{\alpha \in \mathbb{N}_{0}^{N}} i^{|\alpha|} \frac{G^{(\alpha)}(0)}{\alpha !} f^{(\alpha)}(x) .
$$


Observe that the Fourier transform of $T_{G}$ is

$$
\begin{aligned}
\widehat{T_{G}}(z) & =T_{G}\left(e^{-i z \cdot}\right) \\
& =\sum_{\alpha \in \mathbb{N}_{0}^{N}}(-i)^{|\alpha|} \frac{G^{(\alpha)}(0)}{\alpha !}(-i z)^{\alpha} \\
& =\sum_{\alpha \in \mathbb{N}_{0}^{N}} \frac{G^{(\alpha)}(0)}{\alpha !}(-z)^{\alpha} \\
& =G(-z) .
\end{aligned}
$$

The ultradifferential operator $G(D)$ is called elliptic if $G(D)^{-1}\left(\mathcal{A}\left(\mathbb{R}^{N}\right)\right) \subset \mathcal{A}\left(\mathbb{R}^{N}\right)$, where $\mathcal{A}\left(\mathbb{R}^{N}\right)$ denotes the class of real analytic functions on $\mathbb{R}^{N}$. It is said to be $(\omega)$-hypoelliptic if $G(D)^{-1}\left(\mathcal{E}_{(\omega)}\left(\mathbb{R}^{N}\right)\right) \subset$ $\mathcal{E}_{(\omega)}\left(\mathbb{R}^{N}\right)$. The ellipticity and the $(\omega)$-hypoellipticity of an ultradifferential operator are characterized in terms of the distribution of zeros and the growth of the entire function $G(z)$ (see [25] and [14]). We will say that the $(\omega)$-hypoelliptic ultradifferential operator $G(D)$ is strongly $(\omega)$-hypoelliptic if there is a constant $C>0$ such that

$$
C \omega(|x|) \leq \log |G(x)|, x \in \mathbb{R}^{N} .
$$

The existence of strongly $(\omega)$-hypoelliptic ultradifferential operators follows from $[21,49]$.

In order to define ultradifferential (of class $\{\omega\}$ ) operators on the class $\mathcal{D}_{\{\omega\}}^{\prime}\left(\mathbb{R}^{N}\right)$ we can proceed as above imposing that $\log |G(z)|=o(\omega(|z|))$ as $|z|$ tends to infinity.

\subsection{Fréchet spaces invariant under ultradifferential operators}

We want to extend the result of Langenbruch and Voigt to the ultradifferentiable setting. As we already have mentioned before, the space of ultradistributions of Roumieu type $E:=\mathcal{D}_{\{\omega\}}^{\prime}\left(\mathbb{R}^{N}\right)$ is a Fréchet space which is stable under differential operators but which is not contained in $\mathcal{E}_{(\omega)}\left(\mathbb{R}^{N}\right)$.

Moreover, following the arguments of [14, Proposition 2.13], one can construct an $(\omega)$-hypoelliptic ultradifferential operator $G(D)$ such that

$$
G(D)\left(\mathcal{D}_{\{\omega\}}^{\prime}\left(\mathbb{R}^{N}\right)\right) \subset \mathcal{D}_{\{\omega\}}^{\prime}\left(\mathbb{R}^{N}\right) \text {. }
$$

That means that a Fréchet space which is invariant under an $(\omega)$-hypoelliptic ultradifferential operator is not necessary formed by ultradifferentiable functions. 
Note that $G(D)$ strongly $(\omega)$-hypoelliptic implies

$$
G(D)\left(\mathcal{D}_{\{\omega\}}^{\prime}\left(\mathbb{R}^{N}\right)\right) \nsubseteq \mathcal{D}_{\{\omega\}}^{\prime}\left(\mathbb{R}^{N}\right) .
$$

Otherwise, $G(D)$ defines a continuous operator $G(D): \mathcal{D}_{\{\omega\}}^{\prime}\left(\mathbb{R}^{N}\right) \rightarrow$ $\mathcal{D}_{\{\omega\}}^{\prime}\left(\mathbb{R}^{N}\right)$ whose transposed operator $G(-D): \mathcal{D}_{\{\omega\}}\left(\mathbb{R}^{N}\right) \rightarrow \mathcal{D}_{\{\omega\}}\left(\mathbb{R}^{N}\right)$ is continuous. Then, $\log |G(z)|=o(\omega(|z|))$ which is a contradiction with the growth condition of strongly $(\omega)$-hypoelliptic operator.

Next result is an extension of [50, Theorem 1] to the ultradifferentiable setting.

Theorem 4.2.1. Let $E$ be a Fréchet space which is continuously included in $\mathcal{D}_{(\omega)}^{\prime}\left(\mathbb{R}^{N}\right)$ and such that $G(D) E \subset E$ for some strongly $(\omega)$-hypoelliptic ultradifferential operator $G(D)$ of class $(\omega)$. Then $E \subset \mathcal{E}_{(\omega)}\left(\mathbb{R}^{N}\right)$ with continuous inclusion.

Proof. We need some preparation. Let $\left(K_{j}\right)$ denote the closed ball centered at the origin and with radius $j$ and

$X_{j}:=\left\{\mu \in \mathcal{E}_{(\omega)}^{\prime}\left(\mathbb{R}^{N}\right) ; \operatorname{supp} \mu \subset K_{j},\|\mu\|_{j}^{*}:=\sup _{z \in \mathbb{C}^{N}}|\widehat{\mu}(z)| e^{-j \omega(|z|)-j|\operatorname{Im} z|}<\infty\right\}$,

where $\widehat{\mu}$ denotes the Fourier-Laplace transform of $\mu$ (see [22] and Theorem 1.0.17). Then $X_{j}$ is a Banach space and

$$
\mathcal{E}_{(\omega)}^{\prime}\left(\mathbb{R}^{N}\right)=\operatorname{ind}_{j \rightarrow} X_{j}
$$

For every $j \in \mathbb{N}$, the bilinear form

$$
B: E \times \mathcal{D}_{(\omega)}\left(K_{j+1}\right) \rightarrow \mathbb{C}, B(h, \varphi):=\langle h, \varphi\rangle,
$$

is separately continuous, hence it is continuous. Consequently, if we fix a fundamental system of seminorms $\left(p_{m}\right)$ of $E$ then there are constants $C_{j}>0$ and $m_{j} \in \mathbb{N}$, such that

$$
|\langle h, \varphi\rangle| \leq C_{j} p_{m_{j}}(h)|\varphi|_{m_{j}} \forall h \in E, \varphi \in \mathcal{D}_{(\omega)}\left(K_{j+1}\right),
$$

where

$$
|\varphi|_{m}:=\sup _{x \in \mathbb{R}^{N}} \sup _{\alpha \in \mathbb{N}_{0}^{N}}\left|\varphi^{(\alpha)}(x)\right| \exp \left(-m \varphi^{*}\left(\frac{|\alpha|}{m}\right)\right) .
$$

According to property $(\alpha)$ of weight function, there is a constant $L \in \mathbb{N}$ such that

$$
\omega(e t) \leq L(1+\omega(t)) \forall t \geq 0 .
$$


By assumption there is a constant $C>0$ with $C \omega(|x|) \leq \log |G(x)|$ for every $x \in \mathbb{R}^{N}$. Now, for each $j$ we define $G_{j}(z)$ as a suitable power of $G(z)$ such that

$$
\left(1+j+2 m_{j} L\right) \omega(|x|) \leq \log \left|G_{j}(x)\right|, x \in \mathbb{R}^{N}
$$

Then $G_{j}(D)$ is an ultradifferential operator of $(\omega)$-class which is strongly ( $\omega)$-hypoelliptic. Let now $\psi_{j} \in \mathcal{D}_{(\omega)}\left(\stackrel{\circ}{K}_{j+1}\right)$ be a test function which is constant $\psi_{j}=1$ on a neighborhood of $K_{j}$. For any $\mu \in X_{j}$ we define

$$
f(x)=\frac{1}{(2 \pi)^{N}} \int_{\mathbb{R}^{N}} \frac{\widehat{\mu}(t)}{G_{j}(t)} e^{i<x, t>} d t .
$$

Then we can decompose

$$
\mu=G_{j}(-D) f=G_{j}(-D)\left(\psi_{j} f\right)+G_{j}(-D)\left(\left(1-\psi_{j}\right) f\right) .
$$

Moreover, we can apply (4.2) and

$$
\left|t^{\alpha}\right| e^{-m \omega(t)} \leq \exp \left(m \varphi^{*}\left(\frac{|\alpha|}{m}\right)\right) \forall m \in \mathbb{N}, \alpha \in \mathbb{N}_{0}^{N}
$$

to conclude that $f \in \mathcal{C}^{\infty}\left(\mathbb{R}^{N}\right)$ and

$$
|f|_{2 L m_{j}}:=\sup _{\alpha \in \mathbb{N}_{0}^{N}} \sup _{x \in \mathbb{R}^{N}}\left|f^{(\alpha)}(x)\right| \exp \left(-2 m_{j} L \varphi^{*}\left(\frac{|\alpha|}{2 m_{j} L}\right)\right) \leq D_{j}|| \mu \|_{j}^{*}
$$

for some constant $D_{j}>0$ which does not depend on $\mu$. Our aim is to prove that each ultradistribution $h \in E$ can be extended to a continuous and linear map

$$
T_{h}: \mathcal{E}_{(\omega)}^{\prime}\left(\mathbb{R}^{N}\right) \rightarrow \mathbb{C} .
$$

First we claim that the linear map

$$
\Phi: X_{j} \rightarrow \mathcal{E}_{(\omega)}\left(\mathbb{R}^{N}\right), \mu \mapsto\left(1-\psi_{j}(x)\right) \frac{1}{(2 \pi)^{N}} \int_{\mathbb{R}^{N}} \frac{\widehat{\mu}(t)}{G_{j}(t)} e^{i<x, t>} d t
$$

is well-defined and continuous. In fact, since $G_{j}(-D)$ is an $(\omega)$-hypoelliptic operator and $G_{j}(-D) f=\mu$ we have, for every $\mu \in X_{j}$,

$$
\operatorname{sing}_{(\omega)} \operatorname{supp} f \subset \operatorname{sing}_{(\omega)} \operatorname{supp} \mu \subset K_{j},
$$

hence

$$
\left(1-\psi_{j}\right) f \in \mathcal{E}_{(\omega)}\left(\mathbb{R}^{N}\right)
$$


On the other hand, from the convexity of $\varphi^{*}$ and (4.1) we get, for every $x \in \mathbb{R}^{N}$ and $\alpha \in \mathbb{N}_{0}^{N}$,

$$
\begin{aligned}
\left|\left(\psi_{j} f\right)^{(\alpha)}(x)\right| & \leq \sum_{\beta \leq \alpha}\left(\begin{array}{c}
\alpha \\
\beta
\end{array}\right)\left|\psi_{j}^{(\beta)}(x)\right|\left|f^{(\alpha-\beta)}(x)\right| \\
& \leq|f|_{2 L m_{j}}\left|\psi_{j}\right|_{2 L m_{j}} \exp \left(\alpha+2 L m_{j} \varphi^{*}\left(\frac{|\alpha|}{2 L m_{j}}\right)\right) \\
& \leq|f|_{2 L m_{j}}\left|\psi_{j}\right|_{2 L m_{j}} \exp \left(2 m_{j} \varphi^{*}\left(\frac{|\alpha|}{2 m_{j}}\right)+m_{j}\right)
\end{aligned}
$$

and, consequently

$$
\left|\psi_{j} f\right|_{2 m_{j}} \leq e^{m_{j}}|f|_{2 L m_{j}}\left|\psi_{j}\right|_{2 L m_{j}} \leq D_{j} e^{m_{j}}\left|\psi_{j}\right|_{2 L m_{j}} \| \mu||_{j}^{*}
$$

Moreover, it follows from the previous estimates that $\Phi: X_{j} \rightarrow \mathcal{D}_{(\omega)}^{\prime}\left(\mathbb{R}^{N}\right)$ is weakly continuous, hence $\Phi: X_{j} \rightarrow \mathcal{E}_{(\omega)}\left(\mathbb{R}^{N}\right)$ is continuous by the closed graph theorem and the claim is proved.

We now fix $h \in E$ and consider a regularizing family $\left(\eta_{\epsilon}\right)_{\epsilon \downarrow 0}, \eta_{\epsilon} \in$ $\mathcal{D}_{(\omega)}\left(\mathbb{R}^{N}\right)$. Define

$$
T_{j}: X_{j} \rightarrow \mathbb{C}
$$

by

$$
T_{j}(\mu)=\lim _{\epsilon \rightarrow 0}\left\langle G_{j}(D) h,\left(\psi_{j} f\right) * \eta_{\epsilon}\right\rangle+\left\langle h, G_{j}(-D)\left(\left(1-\psi_{j}\right) f\right)\right\rangle,
$$

where

$$
f(x)=\frac{1}{(2 \pi)^{N}} \int_{\mathbb{R}^{N}} \frac{\widehat{\mu}(t)}{G_{j}(t)} e^{i<x, t>} d t .
$$

Let us prove that $T_{j}$ is a well-defined linear map. Since $G_{j}(D) h \in E$ and $\left(\psi_{j} f\right) * \eta_{\epsilon} \in \mathcal{D}_{(\omega)}\left(K_{j+1}\right)$ we get

$$
\left|\left\langle G_{j}(D) h,\left(\psi_{j} f\right) *\left(\eta_{\epsilon_{1}}-\eta_{\epsilon_{2}}\right)\right\rangle\right| \leq C_{j} p_{m_{j}}\left(G_{j}(D) h\right)\left|\left(\psi_{j} f\right) *\left(\eta_{\epsilon_{1}}-\eta_{\epsilon_{2}}\right)\right|_{m_{j}} .
$$

On the other hand, using (4.3), we have

$$
\lim _{\epsilon \rightarrow 0}\left|\left(\psi_{j} f\right) * \eta_{\epsilon}-\left(\psi_{j} f\right)\right|_{m_{j}}=0,
$$

from where it follows that

$$
\left(\left\langle G_{j}(D) h,\left(\psi_{j} f\right) * \eta_{\epsilon}\right\rangle\right)_{\epsilon \downarrow 0}
$$

is a Cauchy net. On the other hand,

$$
G_{j}(-D)\left(\left(1-\psi_{j}\right) f\right)=\mu-G_{j}(-D)\left(\psi_{j} f\right)
$$


4.3 Iterates and Fréchet spaces invariant under partial differential operators

is compactly supported, hence

$$
G_{j}(-D)\left(\left(1-\psi_{j}\right) f\right) \in \mathcal{D}_{(\omega)}\left(\mathbb{R}^{N}\right) .
$$

Consequently $T_{j}$ is a well-defined linear map. From (4.3) and the continuity of

$$
G_{j}(-D) \circ \Phi: X_{j} \rightarrow \mathcal{D}_{(\omega)}\left(K_{j+1}\right)
$$

we conclude that there is a positive constant $M_{j}$ such that

$$
\begin{aligned}
\left|T_{j}(\mu)\right| & \leq C_{j} p_{m_{j}}\left(G_{j}(D) h\right)\left|\psi_{j} f\right|_{m_{j}}+\left|\left\langle h,\left(G_{j}(D) \circ \Phi\right) \mu\right\rangle\right| \\
& \leq M_{j}|| \mu \|_{j}^{*},
\end{aligned}
$$

which proves that

$$
T_{j}: X_{j} \rightarrow \mathbb{C}
$$

is a continuous and linear form. Moreover, for $\mu \in X_{j} \cap \mathcal{D}_{(\omega)}\left(\mathbb{R}^{N}\right)$ we have

$$
\begin{aligned}
T_{j}(\mu) & =\lim _{\epsilon \rightarrow 0}\left(\left\langle h, G_{j}(-D)\left(\psi_{j} f\right) * \eta_{\epsilon}\right\rangle\right. \\
& \left.+\left\langle h, G_{j}(-D)\left(\left(1-\psi_{j}\right) f\right) * \eta_{\epsilon}\right\rangle\right) \\
& =\lim _{\epsilon \rightarrow 0}\left\langle h, G_{j}(-D) f * \eta_{\epsilon}\right\rangle \\
& =\lim _{\epsilon \rightarrow 0}\left\langle h, \mu * \eta_{\epsilon}\right\rangle=\langle h, \mu\rangle .
\end{aligned}
$$

Since the restriction of $T_{j+1}$ to $X_{j}$ coincides with $T_{j}$, we finally conclude that there is a continuous and linear form $T: \mathcal{E}_{(\omega)}^{\prime}\left(\mathbb{R}^{N}\right) \rightarrow \mathbb{C}$ with the property that $T(\mu)=\langle h, \mu\rangle$ for all $\mu \in \mathcal{D}_{(\omega)}\left(\mathbb{R}^{N}\right)$. That is, as ultradistributions,

$$
h=T \in \mathcal{E}_{(\omega)}\left(\mathbb{R}^{N}\right) .
$$

\subsection{Iterates and Fréchet spaces invariant under par- tial differential operators}

In this section we are interested in the following question: let $E \subset$ $\mathcal{D}_{(\omega)}^{\prime}\left(\mathbb{R}^{N}\right)$ be a Fréchet space and $P(D)$ a differential operator of finite order such that $P(D) E \subset E$. We want to look for additional conditions in 
order to ensure that $E \subset \mathcal{E}_{(\omega)}\left(\mathbb{R}^{N}\right)$.

This condition consists precisely in involving extra equicontinuity assumptions on the iterates of $P$ acting on the Fréchet space $E$.

Definition 4.3.1. Let $E$ be a Fréchet space such that $E \subset \mathcal{D}_{(\omega)}^{\prime}\left(\mathbb{R}^{N}\right)$ with continuous inclusion and let $P(D)$ be a differential operator of degree $m$. Then $E$ is said to be $(\omega, P(D))$-stable if $P(D) E \subset E$ and, moreover, for every $k \in \mathbb{N}$, the sequence of operators

$$
P^{j}(D) e^{-k \varphi^{*}\left(m \frac{j}{k}\right)}: E \rightarrow E
$$

is equicontinuous, that is, for every $k \in \mathbb{N}$ and every continuous seminorm $r$ on $E$ there is a continuous seminorm $s$ on $E$ with

$$
r\left(P^{j}(D) f\right) \leq e^{k \varphi^{*}\left(m_{k}^{j}\right)} s(f) \forall j \in \mathbb{N}, f \in E .
$$

Example 4.3.2. We put $\omega_{m}(t)=\omega\left(t^{\frac{1}{m}}\right)$. For every hypoelliptic polynomial $P$ and for every $m \in \mathbb{N}$ the Fréchet space $\mathcal{E}_{P,\left(\omega_{m}\right)}\left(\mathbb{R}^{N}\right)$ is $(\omega, P(D))$-stable.

In the limit case $\omega(t)=\log (1+t),(\omega, P(D))$-stability simply means that $P(D) E \subset E$.

Given a differential operator $P(D)$ and an $(\omega, P(D))$-stable Fréchet space $E \subset \mathcal{D}_{(\omega)}^{\prime}\left(\mathbb{R}^{N}\right)$, we want to analyze whether $E$ consists of smooth functions or even $E \subset \mathcal{E}_{(\lambda)}\left(\mathbb{R}^{N}\right)$ for some weight $\lambda$ related to $\omega$. We observe that, for an arbitrary polynomial $P(D)$, the space

$$
E:=\left\{S \in \mathcal{D}_{\{\omega\}}^{\prime}\left(\mathbb{R}^{N}\right): P(D) S=0\right\},
$$

consisting of the ultradistributions of Roumieu type in the Kernel of $P(D)$, is an $(\omega, P(D))$-stable Fréchet space. Hence in order to have a positive answer to the previous question the polynomial $P$ has to be hypoelliptic.

As proved in Theorem 3.1.7, for any elliptic polynomial $P$ of degree $m$ the space $\mathcal{E}_{P,\left(\omega\left(t^{\frac{1}{m}}\right)\right)}\left(\mathbb{R}^{N}\right)$ is contained in $\mathcal{E}_{(\omega)}\left(\mathbb{R}^{N}\right)$. This fact and the result by Langenbruch and Voigt [50, Theorem 1] permit us to prove a similar result for arbitrary $(\omega, P(D))$-stable Fréchet spaces of distributions.

Proposition 4.3.3. Let $P(D)$ be an elliptic differential operator of degree $m$. If the Fréchet space $E \subset \mathcal{D}^{\prime}\left(\mathbb{R}^{N}\right)$ is $(\omega, P(D))$-stable then $E \subset \mathcal{E}_{(\omega)}\left(\mathbb{R}^{N}\right)$ with continuous inclusion. 
4.3 Iterates and Fréchet spaces invariant under partial differential operators

Proof. According to Langenbruch, Voigt [50, Theorem 1], $E$ is continuously included in $\mathcal{C}^{\infty}\left(\mathbb{R}^{N}\right)$. Hence, for any $f \in E, k \in \mathbb{N}$ and $K \subset \mathbb{R}^{N}$,

$$
\sup _{j \in \mathbb{N}_{0}}\left\|P^{j}(D) f\right\|_{2, K} e^{-k \varphi^{*}\left(m \frac{j}{k}\right)}<\infty,
$$

which means that

$$
f \in \mathcal{E}_{P,\left(\omega\left(t^{\frac{1}{m}}\right)\right)}\left(\mathbb{R}^{N}\right) .
$$

Since $P$ is an elliptic operator, we can apply Theorem 3.1.7 to conclude that $f \in \mathcal{E}_{(\omega)}\left(\mathbb{R}^{N}\right)$.

This result can be partially extended to Fréchet spaces of ultradistributions.

Theorem 4.3.4. Let $P(D)$ be an elliptic differential operator of degree $m$ such that its principal part has real coefficients. If the Fréchet space $E \subset \mathcal{D}_{(\omega)}^{\prime}\left(\mathbb{R}^{N}\right)$ is $(\omega, P(D))$-stable then $E \subset \mathcal{E}_{(\omega)}\left(\mathbb{R}^{N}\right)$ with continuous inclusion.

Proof. We will see that $E$ is invariant under the action of a strongly $(\omega)$ hypoelliptic operator and then the conclusion follows applying Theorem 4.2.1. Throughout the proof, in order to simplify the notation, we will put $\sigma:=\omega_{m}$.

According to $[49$, Corollary 1.4] there are an entire function $g \in H(\mathbb{C})$ without zeros on the real line and a conic neighborhood $\Gamma$ of $\mathbb{R} \backslash\{0\}$, defined by $|\operatorname{Im} z|<\epsilon|\operatorname{Re} z|$, such that

$$
|g(z)| \leq A e^{B \sigma(|z|)} \forall z \in \mathbb{C} \text { and }|g(z)| \geq a e^{b \sigma(|z|)} \forall z \in \Gamma .
$$

We now put

$$
P=Q+P_{m}
$$

where $P_{m}$ is the principal part of $P$ and $Q$ is a polynomial of degree at most $m-1$. For each $\xi \in \mathbb{R}^{n}$ we have, for some $d>0$ and for $|\xi|$ large enough,

$$
\begin{aligned}
\left|\frac{\operatorname{Im} P(\xi)}{\operatorname{Re} P(\xi)}\right| & \leq \frac{|Q(\xi)|}{\left|P_{m}(\xi)\right|-|Q(\xi)|} \\
& \leq \frac{|Q(\xi)|}{d|\xi|^{m}-|Q(\xi)|} .
\end{aligned}
$$

Hence, there is $R>0$ such that $P(\xi) \in \Gamma$ whenever $\xi \in \mathbb{R}^{N}$ and $|\xi| \geq R$. We now define $G \in H\left(\mathbb{C}^{N}\right)$ by

$$
G(z):=g\left(\epsilon_{0} P(z)\right)
$$


where $\epsilon_{0}>0$ is such that $G(\xi) \neq 0$ for every $\xi \in \mathbb{R}^{N}$ with $|\xi| \leq R$. Hence $G(\xi) \neq 0$ for every $\xi \in \mathbb{R}^{N}$. Then, for some constants $C, D>0$, we have

$$
\begin{aligned}
|G(z)| & \leq A e^{B \sigma\left(\epsilon_{0}|P(z)|\right)} \leq C e^{D \sigma\left(|z|^{m}\right)} \\
& =C e^{D \omega(|z|)}
\end{aligned}
$$

for every $z \in \mathbb{C}^{N}$. On the other hand, there is $\delta>0$ with $|P(\xi)| \geq \delta|\xi|^{m}$ for $|\xi|$ large enough. Consequently, there is $q \in \mathbb{N}$ such that

$$
\begin{aligned}
|G(\xi)| & \geq a e^{b \sigma\left(\epsilon_{0}|P(\xi)|\right)} \\
& \geq a e^{b \omega\left(2^{-q}|\xi|\right)}
\end{aligned}
$$

for each $\xi \in \mathbb{R}^{N}$ with $|\xi|$ large enough. Since $G$ does not vanish on $\mathbb{R}^{N}$, we finally deduce

$$
|G(\xi)| \geq a^{\prime} e^{b^{\prime} \omega(|\xi|)}
$$

for $|\xi| \in \mathbb{R}^{N}$. To conclude that $G(D)$ is a strongly $(\omega)$-hypoelliptic ultradifferential operator of $(\omega)$-class it is enough to show that (see [14, Theorem 2.1])

$$
\lim _{\substack{|z| \rightarrow \infty \\ G(z)=0}} \frac{|\operatorname{Im} z|}{\omega(|z|)}=\infty
$$

We are going to prove that each $z \in \mathbb{C}^{N}$ with $|\operatorname{Im} z| \leq A \omega(|z|)$ also satisfies $|\operatorname{Im} P(z)|<\varepsilon|\operatorname{Re} P(z)|$ provided that $|z|$ is big enough, and consequently $G(z)=g(P(z)) \neq 0$. Since $\omega(t)=o(t)$ we may assume $|\operatorname{Im} z| \leq$ $A \omega(|\operatorname{Re} z|)$ for some different constant $A$. Clearly,

$$
|\operatorname{Im} Q(z)| \leq|Q(z)| \leq C|z|^{m-1} \leq C(1+A)^{m}|\operatorname{Re} z|^{m-1} .
$$

On the other hand, by Taylor formula

$$
P_{m}(z)=P_{m}(\operatorname{Re} z+i \operatorname{Im} z)=P_{m}(\operatorname{Re} z)+\sum_{\alpha \neq 0} \frac{P_{m}^{(\alpha)}(\operatorname{Re} z)}{\alpha !}(i \operatorname{Im} z)^{\alpha} .
$$

Each term in the sum above is estimated as follows

$$
\left|P_{m}^{(\alpha)}(\operatorname{Re} z)(i \operatorname{Im} z)^{\alpha}\right| \leq|\operatorname{Re} z|^{m-|\alpha|}|\operatorname{Im} z|^{|\alpha|} .
$$

Therefore, for $|z|$ big enough, the whole sum is not bigger than $D|\operatorname{Re} z|^{m-1}|\operatorname{Im} z|$. Since the principal part of $P$ has real coefficients we finally have

$$
|\operatorname{Im} P(z)| \leq D^{\prime}|\operatorname{Re} z|^{m-1}|\operatorname{Im} z| \leq D^{\prime} A|\operatorname{Re} z|^{m-1} \omega(|\operatorname{Re} z|)
$$


4.3 Iterates and Fréchet spaces invariant under partial differential operators

whereas

$$
\begin{aligned}
|\operatorname{Re} P(z)| & \geq\left|P_{m}(\operatorname{Re} z)\right|-D^{\prime} A|\operatorname{Re} z|^{m-1} \omega(|\operatorname{Re} z|) \\
& \geq d|\operatorname{Re} z|^{m}-D^{\prime} A|\operatorname{Re} z|^{m-1} \omega(|\operatorname{Re} z|) \\
& \geq L|\operatorname{Re} z|^{m} .
\end{aligned}
$$

Hence, it is clear that

$$
|\operatorname{Im} P(z)|<\varepsilon|\operatorname{Re} P(z)|
$$

provided that $|z|$ is big enough.

We now check that $G(D) E \subset E$. Since the entire function

$$
g(z)=\sum_{j \in \mathbb{N}_{0}} a_{j} z^{j}
$$

satisfies (4.5), we can use the convexity of $\varphi^{*}$ and Cauchy inequalities to find $k \in \mathbb{N}$ and $C>0$ such that

$$
\left|a_{j}\right| \leq C e^{-k \varphi^{*}\left(m_{k}^{j}\right)} \forall j \in \mathbb{N}_{0} .
$$

Moreover,

$$
G(D)=\sum_{j \in \mathbb{N}_{0}} a_{j} P^{j}(D)
$$

If $r$ is a continuous seminorm on $E$ then there is another continuous seminorm $s$ on $E$ such that, for every $f \in E$,

$$
\begin{aligned}
\sum_{j \in \mathbb{N}_{0}}\left|a_{j}\right| r\left(P^{j}(D) f\right) & \leq C D \sup _{j \in \mathbb{N}_{0}} e^{-2 k \varphi^{*}\left(m \frac{j}{2 k}\right)} r\left(P^{j}(D) f\right) \\
& \leq C D s(f)
\end{aligned}
$$

where

$$
D=\sum_{j \in \mathbb{N}_{0}} \frac{e^{2 k \varphi^{*}\left(m \frac{j}{2 k}\right)}}{e^{k \varphi^{*}\left(m \frac{j}{k}\right)}}<+\infty .
$$

This proves that the series $\sum_{j \in \mathbb{N}_{0}} a_{j} P^{j}(D) f$ converges in the Fréchet space $E$ and $G(D) f \in E$.

Remark 4.3.5. If $E$ is invariant under complex conjugation, the assumption that the principal part of $P$ should be real is redundant. Indeed, $(\omega, P(D))$ stability then implies $(\omega, Q(D))$-stability where $Q(D)=P(D) \overline{P(D)}=$ $P(D) \bar{P}(-D)$ and the principal part of $Q$ has real coefficients. 
According to Condition IIb of [33, II, Theorem 11.1.3], for every hypoelliptic differential operator $P(D)$ of degree $m$, there are $c>0$ and $0<r<1$ such that $|P(\xi)| \geq c|\xi|^{r m}$ and $\left|P^{(\alpha)}(\xi)\right| \leq c^{-1}|P(\xi)||\xi|^{-r|\alpha|}$ if $\xi \in \mathbb{R}^{N}$ and $|\xi|$ is large enough. Let us take a weight function $\omega$ such that $\lim _{t \rightarrow \infty} \frac{\omega(t)}{t^{r}}=$ 0 . In the sequel we will consider $\lambda(t):=\omega\left(t^{r}\right)$.

Proposition 4.3.6. We assume that the hypoelliptic differential operator $P(D)$ of degree $m$ satisfies

$$
\lim _{x \in \mathbb{R}^{N},|x| \rightarrow \infty} \frac{\operatorname{Im} P(x)}{\operatorname{Re} P(x)}=0 .
$$

If the Fréchet space $E \subset \mathcal{D}_{(\lambda)}^{\prime}\left(\mathbb{R}^{N}\right)$ is $(\omega, P(D))$-stable then $E \subset \mathcal{E}_{(\lambda)}\left(\mathbb{R}^{N}\right)$ with continuous inclusion.

Proof. Let $\left(K_{j}\right)$ denote the closed ball centered at the origin and with radius $j$ and

$$
X_{j}:=\left\{\mu \in \mathcal{E}_{(\lambda)}^{\prime}\left(\mathbb{R}^{N}\right) ; \operatorname{supp} \mu \subset K_{j},\|\mu\|_{j}^{*}:=\sup _{z \in \mathbb{C}^{N}}|\widehat{\mu}(z)| e^{-j \lambda(|z|)-j|\operatorname{Im} z|}<\infty\right\} .
$$

Then $X_{j}$ is a Banach space and

$$
\mathcal{E}_{(\lambda)}^{\prime}\left(\mathbb{R}^{N}\right)=\operatorname{ind}_{j \rightarrow} X_{j}
$$

For every $j \in \mathbb{N}$, the bilinear form

$$
B: E \times \mathcal{D}_{(\lambda)}\left(K_{j+1}\right) \rightarrow \mathbb{C}, B(h, \varphi):=\langle h, \varphi\rangle,
$$

is separately continuous, hence it is continuous. Consequently, if we fix a fundamental system of seminorms $\left(p_{m}\right)$ of $E$ there are constants $C_{j}>0$ and $m_{j} \in \mathbb{N}$, such that

$$
|\langle h, \varphi\rangle| \leq C_{j} p_{m_{j}}(h)|\varphi|_{m_{j}} \forall h \in E, \varphi \in \mathcal{D}_{(\lambda)}\left(K_{j+1}\right),
$$

where

$$
|\varphi|_{m}:=\sup _{x \in \mathbb{R}^{N}} \sup _{\alpha \in \mathbb{N}_{0}^{N}}\left|\varphi^{(\alpha)}(x)\right| \exp \left(-m \varphi_{\lambda}^{*}\left(\frac{|\alpha|}{m}\right)\right) .
$$

As in the proof of Theorem 4.3.4, there are an entire function $g \in H(\mathbb{C})$ without zeros on the real line and a conic neighborhood $\Gamma$ of $\mathbb{R} \backslash\{0\}$, defined by $|\operatorname{Im} z|<\epsilon|\operatorname{Re} z|$, such that

$$
|g(z)| \leq A e^{B \omega\left(|z|^{\frac{1}{m}}\right)} \forall z \in \mathbb{C} \text { and }|g(z)| \geq a e^{b \omega\left(|z|^{\frac{1}{m}}\right)} \forall z \in \Gamma .
$$


4.3 Iterates and Fréchet spaces invariant under partial differential operators

We now define $G \in H\left(\mathbb{C}^{N}\right)$ by $G(z):=g(P(z))$ and we check that $G(D)$ is $(\omega)$-hypoelliptic. In fact, for some constants $C, D>0$,

$$
|G(z)| \leq A e^{B \omega\left(|P(z)|^{\frac{1}{m}}\right)} \leq C e^{D \omega(|z|)}
$$

for every $z \in \mathbb{C}^{N}$ and

$$
|G(\xi)| \geq a e^{b \omega\left(|\xi|^{r}\right)}=a e^{b \lambda(|\xi|)}
$$

for some $a, b>0$ and every $\xi \in \mathbb{R}^{N}$. We now check that

$$
\lim _{\substack{|z| \rightarrow \infty \\ G(z)=0}} \frac{|\operatorname{Im} z|}{\omega(|z|)}=\infty
$$

To this end we observe that $G(z)=0$ implies $|\operatorname{Im} P(z)| \geq \epsilon|\operatorname{Re} P(z)|$. Hence, it suffices to prove that, for any $A>0$, the inequality $|\operatorname{Im} z|<$ $A \omega(|z|)$ implies $|\operatorname{Im} P(z)|<\epsilon|\operatorname{Re} P(z)|$ whenever $|z|$ is large enough. Since $\omega(t)=o(t)$ we may assume $|\operatorname{Im} z| \leq A \omega(|\operatorname{Re} z|)$ for some different constant $A$. Using Taylor's formula we have, for $z=x+i y, x, y \in \mathbb{R}^{N}$,

$$
P(z)=P(x)+\sum_{\alpha \neq 0} \frac{P^{(\alpha)}(x)}{\alpha !}(i y)^{(\alpha)} .
$$

Now, since $P$ is hypoelliptic get, for some positive constant $C$ (which depends on $A$ ),

$$
\left|\sum_{\alpha \neq 0} \frac{P^{(\alpha)}(x)}{\alpha !}(i y)^{(\alpha)}\right| \leq C|P(x)| \sum_{\alpha \neq 0} \frac{1}{\alpha !}\left(\frac{\omega(|x|)}{|x|^{r}}\right)^{|\alpha|} .
$$

Therefore, using $\lim _{t \rightarrow \infty} \frac{\omega(t)}{t^{r}}=0$ and $\lim _{x \in \mathbb{R}^{N},|x| \rightarrow \infty} \frac{\operatorname{Im} P(x)}{\operatorname{Re} P(x)}=0$ we deduce that

$$
\left|\sum_{\alpha \neq 0} \frac{P^{(\alpha)}(x)}{\alpha !}(i y)^{(\alpha)}\right|<\min \left(\frac{\epsilon}{2}, \frac{1}{2}\right)|\operatorname{Re} P(x)|
$$

for $|x|$ large enough (equivalently, for $|z|$ large enough, since $|y| \leq A \omega(|x|)$ and $\omega(|x|)=o(|x|)$ as $|x|$ tends to $\infty)$. Therefore, for $|z|$ large enough,

$$
|\operatorname{Im} P(z)|<\frac{\epsilon}{2}|\operatorname{Re} P(x)|
$$

while

$$
|\operatorname{Re} P(z)| \geq|\operatorname{Re} P(x)|-\left|\sum_{\alpha \neq 0} \frac{P^{(\alpha)}(x)}{\alpha !}(i y)^{(\alpha)}\right| \geq \frac{1}{2}|\operatorname{Re} P(x)| .
$$


Hence,

$$
|\operatorname{Im} P(z)|<\epsilon|\operatorname{Re} P(z)|,
$$

as we wanted to check. Consequently, $G(D)$ is $(\omega)$-hypoelliptic [14, Theorem 2.1]. Proceeding as in Theorem 4.3.4 we conclude that $G(D) E \subset E$ and $G(D): E \rightarrow E$ is continuous and linear map. To finish the proof, let $\mu \in X_{j}$ be given and take $l_{j} \in \mathbb{N}$ with

$$
b l_{j}-\bar{m}_{j}>j+1
$$

(where $\bar{m}_{j}$ is as large as needed later) and define $G_{j}(D)=G^{l}(D)$ and

$$
f_{j}(x):=\frac{1}{(2 \pi)^{N}} \int_{\mathbb{R}^{N}} \frac{\widehat{\mu}(t)}{G_{j}(t)} e^{i<x, t>} d t .
$$

Since, for every $\alpha \in \mathbb{N}_{0}^{N}$,

$$
\begin{aligned}
\left|\frac{t^{\alpha} \widehat{\mu}(-t)}{G_{j}(t)}\right| & \leq \frac{\|\mu\|_{j}^{*}}{a} e^{\left(-b l_{j}+j\right) \lambda(t)+|\alpha| \log (t)} \\
& \leq \frac{\|\mu\|_{j}^{*}}{a} e^{-\lambda(t)} e^{\bar{m}_{j} \varphi_{\lambda}^{*}\left(\frac{|\alpha|}{\bar{m}_{j}}\right)},
\end{aligned}
$$

then

$$
f_{j} \in \mathcal{C}^{\infty}\left(\mathbb{R}^{N}\right) \subset \mathcal{D}_{(\omega)}^{\prime}\left(\mathbb{R}^{N}\right)
$$

and

$$
\sup _{x \in \mathbb{R}^{N}} \sup _{\alpha \in \mathbb{N}_{0}^{N}}\left|f_{j}^{(\alpha)}(x)\right| e^{-\bar{m}_{j} \varphi_{\lambda}^{*}\left(\frac{|\alpha|}{\bar{m}_{j}}\right)}<+\infty .
$$

Moreover

$$
G_{j}(-D) f_{j}=\mu
$$

in $\mathcal{D}_{(\omega)}^{\prime}\left(\mathbb{R}^{N}\right)$. Let $\psi_{j} \in \mathcal{D}_{(\omega)}\left(\stackrel{\circ}{K}_{j+1}\right)$ be such that $\psi_{j}=1$ on a neighborhood of $K_{j}$. As in Theorem 4.3.4 we will prove that each ultradistribution $h \in E$ can be extended to a continuous and linear map

$$
T_{h}: \mathcal{E}_{(\lambda)}^{\prime}\left(\mathbb{R}^{N}\right) \rightarrow \mathbb{C}
$$

Since $G_{j}(-D)$ is $(\omega)$-hypoelliptic then

$$
\operatorname{sing}_{(\omega)} \operatorname{supp} f_{j} \subset \operatorname{conv}\left(\operatorname{sing}_{(\omega)} \operatorname{supp} f_{j}\right) \subset K_{j}
$$

and the mapping

$$
X_{j} \rightarrow \mathcal{E}_{(\omega)}\left(\mathbb{R}^{N}\right), \mu \mapsto\left(1-\psi_{j}(x)\right) \frac{1}{(2 \pi)^{N}} \int_{\mathbb{R}^{N}} \frac{\widehat{\mu}(t)}{G_{j}(t)} e^{i<x, t>} d t
$$


4.3 Iterates and Fréchet spaces invariant under partial differential operators

is well-defined. Moreover, as in the proof of Theorem 4.2.1, the mapping $X_{j} \rightarrow \mathcal{E}_{(\lambda)}\left(\mathbb{R}^{N}\right)$ is continuous. Hence, by the closed graph theorem, also

$$
X_{j} \rightarrow \mathcal{E}_{(\omega)}\left(\mathbb{R}^{N}\right)
$$

is continuous. We now fix $h \in E$ and consider a regularizing family $\left(\eta_{\epsilon}\right)$ of test functions in $\mathcal{D}_{(\omega)}\left(\mathbb{R}^{N}\right)$. If $\bar{m}_{j}$ is big enough, we may guarantee that

$$
\lim _{\epsilon \rightarrow 0}\left|\left(\psi_{j} f_{j}\right) * \eta_{\epsilon}-\left(\psi_{j} f_{j}\right)\right|_{m_{j}}=0,
$$

from where it follows that

$$
\left(\left\langle G_{j}(D) h,\left(\psi_{j} f_{j}\right) * \eta_{\epsilon}\right\rangle\right)_{\epsilon \downarrow 0}
$$

is a Cauchy net. Define

$$
T_{j}: X_{j} \rightarrow \mathbb{C}
$$

by

$$
T_{j}(\mu)=\lim _{\epsilon \rightarrow 0}\left\langle G_{j}(D) h,\left(\psi_{j} f_{j}\right) * \eta_{\epsilon}\right\rangle+\left\langle h, G_{j}(-D)\left(\left(1-\psi_{j}\right) f_{j}\right)\right\rangle .
$$

The same argument as in Theorem 4.2.1 gives that $T_{j}$ is a continuous linear form, the restriction of $T_{j+1}$ to $X_{j}$ coincides with $T_{j}$ and $T_{j}(\mu)=\langle h, \mu\rangle$ for every $\mu \in X_{j} \cap \mathcal{D}_{(\omega)}\left(\mathbb{R}^{N}\right)$. That is, there is $f \in \mathcal{E}_{(\lambda)}\left(\mathbb{R}^{N}\right)$ such that

$$
\langle f, \mu\rangle=\langle h, \mu\rangle
$$

for every $\mu \in \mathcal{D}_{(\omega)}\left(\mathbb{R}^{N}\right)$. Since $\mathcal{D}_{(\omega)}\left(\mathbb{R}^{N}\right)$ is dense in $\mathcal{D}_{(\lambda)}\left(\mathbb{R}^{N}\right)$ then $h=$ $f \in \mathcal{E}_{(\lambda)}\left(\mathbb{R}^{N}\right)$.

Now, we introduce a more general class of hypoelliptic operators. Let $m=\left(m_{1}, \ldots, m_{N}\right) \in \mathbb{N}^{N}, \alpha=\left(\alpha_{1}, \ldots, \alpha_{N}\right) \in \mathbb{N}_{0}^{N}$ be given and set

$$
|\alpha: m|=\sum_{k=1}^{N} \frac{\alpha_{k}}{m_{k}} .
$$

Let $P(D)=\sum_{\alpha} a_{\alpha} D^{\alpha}$ a partial differential operator. Suppose that $|\alpha: m| \leq$ 1 for all the terms in $P(D)$, then

$$
P(D)=\sum_{|\alpha: m| \leq 1} a_{\alpha} D^{\alpha}
$$

We set $P^{0}(D)=\sum_{|\alpha: m|=1} a_{\alpha} D^{\alpha}$ and we call $P^{0}(D)$ the generalized principal part of $P(D)$ with respect to $m$. 
Definition 4.3.7. If $P^{0}(\xi) \neq 0$ for $0 \neq \xi \in \mathbb{R}^{N}$ the operator $P(D)$ is called semi-elliptic.

According to [33, II, Theorem 11.1.11], every semi-elliptic polynomial is hypoelliptic.

Example 4.3.8. Note that when $m_{k}=m$ for every $k$, the semi-elliptic operators are just the elliptic operators of order $m$. The heat operator and the $p$-parabolic operators of Petrowsky are semi-elliptic.

Corollary 4.3.9. We assume that the differential operator $P(D)$ of degree $m$ satisfies

1. $P(D)$ is hypoelliptic and has real coefficients

or

2. $P(D)$ is semi-elliptic with real generalized principal part.

If the Fréchet space $E \subset \mathcal{D}_{(\lambda)}^{\prime}\left(\mathbb{R}^{N}\right)$ is $(\omega, P(D))$-stable then $E \subset \mathcal{E}_{(\lambda)}\left(\mathbb{R}^{N}\right)$ with continuous inclusion.

Proof. It is straightforward to check that in both cases

$$
\lim _{x \in \mathbb{R}^{N},|x| \rightarrow \infty} \frac{\operatorname{Im} P(x)}{\operatorname{Re} P(x)}=0
$$

Then, Proposition 4.3.6 applies. For the semi-elliptic case use the inequality

$$
\left|\xi^{\alpha}\right| \leq\left(\sum_{k=1}^{N}\left|\xi_{k}\right|^{m_{k}}\right)^{|\alpha: m|}
$$

which is a consequence of $\left|\xi_{k}\right|^{m_{k}} \leq \sum_{k=1}^{N}\left|\xi_{k}\right|^{m_{k}}$.

A weight $\omega$ is called a strong weight if it satisfies the additional condition

$(\epsilon)$ there exists $C \geq 1$ such that for all $y>0$,

$$
\int_{1}^{\infty} \frac{\omega(t y)}{t^{2}} d t \leq C \omega(y)+C
$$

Examples of weight functions with and without property $(\epsilon)$ can be found in [52]. 
4.3 Iterates and Fréchet spaces invariant under partial differential operators

Corollary 4.3.10. Let $\omega$ be a strong weight and $P(D)$ a ( $\omega)$-hypoelliptic differential operator of degree $m$ such that

$$
\lim _{x \in \mathbb{R}^{N},|x| \rightarrow \infty} \frac{\operatorname{Im} P(x)}{\operatorname{Re} P(x)}=0 .
$$

Then $\mathcal{E}_{P,\left(\omega\left(t^{\frac{1}{m}}\right)\right)}\left(\mathbb{R}^{N}\right)$ is continuously contained in $\mathcal{E}_{(\lambda)}\left(\mathbb{R}^{N}\right)$ for some non-quasianalytic weight $\lambda$.

Proof. According to [26,3.7], there is $0<r<1$ such that $P(D)$ is $\left\{t^{r}\right\}$-hypoelliptic and $\lim _{t \rightarrow \infty} \frac{\omega(t)}{t^{r}}=0$. Then Proposition 4.3.6 implies that

$E=\mathcal{E}_{P,\left(\omega\left(t^{\frac{1}{m}}\right)\right)}\left(\mathbb{R}^{N}\right)$ is continuously contained in $\mathcal{E}_{(\lambda)}\left(\mathbb{R}^{N}\right)$ for $\lambda(t):=$ $\omega\left(t^{r}\right)$.

To finish this chapter, we give an alternative proof of Corollary 3.2.2 in the Beurling case.

Lemma 4.3.11. Let $P(D)$ and $Q(D)$ be partial differential operators of order $m_{1}$ and $m_{2}$, respectively. Suppose that $E$ is a Fréchet space which is $(\omega, P(D))$-stable and $(\omega, Q(D))$-stable, then $E$ is also $(\omega, P(D) \circ Q(D))$-stable.

Proof. Note that the order of $(P \circ Q)(D)$ is $m_{1}+m_{2}$. Given $k \in \mathbb{N}$ and given $r$ a continuous seminorm on $E$, the $(\omega, P(D))$-stability gives a seminorm $s$ on $E$ with

$$
r\left((P \circ Q)^{j}(D) f\right) \leq e^{k \varphi^{*}\left(\frac{m_{1} j}{k}\right)} s\left(Q^{j}(D) f\right) \forall j \in \mathbb{N}, f \in E .
$$

Now, since $E$ is $(\omega, Q(D))$-stable, take a seminorm $t$ on $E$ such that

$$
r\left((P \circ Q)^{j}(D) f\right) \leq e^{k \varphi^{*}\left(\frac{m_{1} j}{k}\right)} e^{k \varphi^{*}\left(\frac{m_{2} j}{k}\right)} t(f) \forall j \in \mathbb{N}, f \in E .
$$

Since, $\varphi$ is convex we can deduce the inequality

$$
k \varphi^{*}\left(\frac{m_{1} j}{k}\right)+k \varphi^{*}\left(\frac{m_{2} j}{k}\right) \leq k \varphi^{*}\left(\frac{\left(m_{1}+m_{2}\right) j}{k}\right)
$$

to conclude that $E$ is $(\omega, P(D) \circ Q(D))$-stable.

Corollary 4.3.12. Let $\omega(t)=\log ^{\beta}(1+t), \beta>1$, be given and $P(D)$ a differential operator of degree $m$. Then $\mathcal{E}_{P,(\omega)}\left(\mathbb{R}^{N}\right)=\mathcal{E}_{(\omega)}\left(\mathbb{R}^{N}\right)$ if, and only if, $P$ is hypoelliptic. 
Proof. First, recall that the weight $\omega_{m}(t)=\omega\left(t^{\frac{1}{m}}\right), m \geq 1$, is equivalent to the weight $\omega(t)=\log ^{\beta}(1+t)$. It follows from Corollary 3.2.1 that the hypoellipticity of $P$ is a necessary condition in order to get the identity $\mathcal{E}_{P,(\omega)}\left(\mathbb{R}^{N}\right)=\mathcal{E}_{(\omega)}\left(\mathbb{R}^{N}\right)$. We now assume that $P$ is hypoelliptic and denote $\bar{P}(\xi):=\overline{P(\xi)}$ the conjugate of $P$. The polynomials $P$ and $\bar{P}$ are equally strong, hence we deduce from Corollary 3.1.5 that $\mathcal{E}_{P,(\omega)}\left(\mathbb{R}^{N}\right)=$ $\mathcal{E}_{\bar{P},(\omega)}\left(\mathbb{R}^{N}\right)$. In particular, using the previous Lemma, we have $\mathcal{E}_{P,(\omega)}\left(\mathbb{R}^{N}\right)$ is an $(\omega, P(D) \circ \bar{P}(D))$-stable Fréchet space. Since the hypoelliptic polynomial (of degree $2 m$ ) $P \bar{P}$ has real coefficients we can apply Proposition 4.3.6 to finally conclude that $\mathcal{E}_{P,(\omega)}\left(\mathbb{R}^{N}\right)$ is contained in $\mathcal{E}_{(\omega)}\left(\mathbb{R}^{N}\right)$. The converse inclusion always holds in view of Theorem 2.3.1. 


\section{Chapter 5}

\section{Vector valued}

\section{ultradifferentiable functions of Roumieu type in Fréchet \\ spaces}

In this chapter, vector valued $\omega$-ultradifferentiable functions $f: \Omega \rightarrow$ $E$ in the setting of non-quasianalytic classes in the sense of Braun, Meise and Taylor with values in a locally convex space $E$ are introduced. As in the cases of real analytic or non-quasianalytic curves treated by Kriegl, Michor and Rainer, three possible notions of vector valued ultradifferentiable function are given. We will define weakly ultradifferentiable function and strongly ultradifferentiable function and also the notion of vector valued ultradifferentiable function in a bornologically sense. Our aim is to characterize when these notions coincide in case $E$ is a Fréchet or an (LB)-space.

Like in the case of real analytic functions [9], the topological invariant (DN) of Vogt ([54]) plays an important role. More particularly, we prove in Theorem 5.2.4 that the three definitions above coincide in a Fréchet space $E$ if and only if $E$ satisfies (DN). However, the case of an (LB)-space $E$ behaves different from the real analytic case. This is the content of Theorem 5.2.6.

Our main tools are tensor products [41], the characterizations of the identity $L(E, F)=L B(E, F)[7,63]$ and the modern theory of Fréchet spaces [54].

\subsection{Definitions and properties}


Vector valued ultradifferentiable functions of Roumieu type in Fréchet 78

spaces

From now on, $E$ denotes a sequentially complete locally convex space and $\omega$ a non-quasianalytic weight function. A treatment of $\mathcal{C}^{\infty}$-functions with values in a locally convex space can be seen in Jarchow [34].

Definition 5.1.1. A function $f: \Omega \rightarrow E$ is weakly $\omega$-ultradifferentiable (of Roumieu type), and we denote $f \in \mathcal{E}_{\{\omega\}}(\Omega, E)$, if for all $u \in E^{\prime}, u \circ f \in$ $\mathcal{E}_{\{\omega\}}(\Omega)$, i.e., $\forall u \in E^{\prime}$ and $\forall K \subset \subset \Omega, u \circ f \in \mathcal{C}^{\infty}(\Omega)$ and there exists $m$ such that

$$
\sup _{x \in K} \sup _{\alpha \in \mathbb{N}_{0}}\left|(u \circ f)^{(\alpha)}(x)\right| \exp \left(-\frac{1}{m} \varphi^{*}(|\alpha| m)\right)<\infty .
$$

According to a classical result of Grothendieck [31], a function $f: \Omega \rightarrow$ $E$ belongs to $\mathcal{C}^{\infty}(\Omega, E)$ in a vector sense if and only if for all $u \in E^{\prime}$, $u \circ f \in \mathcal{C}^{\infty}(\Omega)$. As a consequence, it follows $\mathcal{E}_{\{\omega\}}(\Omega, E) \subset \mathcal{C}^{\infty}(\Omega, E)$.

Let $\omega$ be a non-quasianalytic weight function. Denote

$\mathcal{S}_{\omega}:=\left\{\sigma: \sigma\right.$ is a weight function satisfying $\sigma=o(\omega)$, i.e., $\left.\lim _{t \rightarrow \infty} \frac{\sigma(t)}{\omega(t)}=0\right\}$.

By [53, Corollary 3.3], the family of seminorms defined by

$$
\|f\|_{K, \sigma}:=\sup _{x \in K} \sup _{\alpha \in \mathbb{N}_{0}^{N}}\left|f^{(\alpha)}(x)\right| \exp \left(\varphi_{\sigma}^{*}(|\alpha|)\right), f \in \mathcal{E}_{\{\omega\}}(\Omega),
$$

where $K$ is compact subset in $\Omega$ and $\sigma \in \mathcal{S}_{\omega}$, is a fundamental system of seminorms of $\mathcal{E}_{\{\omega\}}(\Omega)$. This result also holds in the quasianalytic case for an open convex subset $\Omega$ by a result due to Bonet, Galbis and Momm (see [16, Lemma 13]).

We recall a regularity condition in inductive limits due to Makarov [51].

Definition 5.1.2. An inductive limit $X=\operatorname{ind}_{n} X_{n}$ is regular if each bounded subset of the inductive limit is contained and bounded in some step $X_{n}$.

A complete LF-space is regular by the Grothendieck Factorization Theorem.

Proposition 5.1.3. Let $E$ be a sequentially complete locally convex space and let $f: \Omega \rightarrow E$. Then, $f \in \mathcal{E}_{\{\omega\}}(\Omega, E)$ if and only if $f \in \mathcal{C}^{\infty}(\Omega, E)$ and for each continuous seminorm $q$ of $E$ and $K$ compact subset in $\Omega$ there exists $m \in \mathbb{N}$ such that

$$
\sup _{x \in K} \sup _{\alpha \in \mathbb{N}_{0}^{N}} q\left(f^{(\alpha)}(x)\right) \exp \left(-\frac{1}{m} \varphi^{*}(|\alpha| m)\right)<\infty .
$$


Proof. Assume $f \in \mathcal{E}_{\{\omega\}}(\Omega, E)$. By the result of Grothendieck, $f \in \mathcal{C}^{\infty}(\Omega, E)$. On the other hand, for all $u \in E^{\prime}, u \circ f \in \mathcal{E}_{\{\omega\}}(\Omega)$. For every weight $\sigma \in \mathcal{S}_{\omega}$, every compact subset $K$ and for all $u \in E^{\prime}$ we have

$$
\sup _{x \in K} \sup _{\alpha \in \mathbb{N}_{0}^{N}}\left|u\left(f^{(\alpha)}(x)\right)\right| \exp \left(-\varphi_{\sigma}^{*}(|\alpha|)\right)<\infty .
$$

Then, the set $\left\{f^{(\alpha)}(x) \exp \left(-\varphi_{\sigma}^{*}(|\alpha|)\right): x \in K, \alpha \in \mathbb{N}_{0}\right\}$ is weakly bounded and hence bounded in $E$. As a consequence, given a continuous seminorm $q$ on $E$, it is clear that the subset of $\mathcal{E}_{\{\omega\}}(\Omega)$ given by

$$
B_{q}:=\left\{u \circ f:|u| \leq q, u \in E^{\prime}\right\}
$$

is bounded in $\mathcal{E}_{\{\omega\}}(\Omega)$. For each compact subset $K$ in $\Omega, B_{q}$ is bounded in $\mathcal{E}_{\{\omega\}}(K)$ which is complete and regular, hence there is $m \in \mathbb{N}$ such that $B_{q}$ is contained and bounded in $\mathcal{E}_{\omega}^{\frac{1}{m}}(K)$, i.e.,

$$
\sup _{g \in B_{q}} p_{K, \frac{1}{m}}(g)<\infty
$$

This and Hahn-Banach's Theorem imply

$$
\sup _{x \in K} \sup _{\alpha \in \mathbb{N}_{0}^{N}} q\left(f^{(\alpha)}(x)\right) \exp \left(-\frac{1}{m} \varphi^{*}(|\alpha| m)\right)=\sup _{g \in B_{q}} p_{K, \frac{1}{m}}(g)<\infty .
$$

We emphasize that in Proposition 5.1.3 the natural number $m$ depends on the seminorm $q$ and the compact set $K$. Compare with the definition below.

Definition 5.1.4. A smooth function $f: \Omega \rightarrow E$ is topologically or strongly $\omega$-ultradifferentiable (of Roumieu type), and we denote $f \in \mathcal{E}_{\{\omega\}}^{t}(\Omega, E)$, if $\forall K \subset \subset \Omega$ there exists $m$ such that $\forall q$ continuous seminorm of $E$

$$
\left.\sup _{x \in K} \sup _{\alpha \in \mathbb{N}_{0}^{N}} q\left(f^{(\alpha)}(x)\right)\right) \exp \left(-\frac{1}{m} \varphi^{*}(m|\alpha|)\right)<\infty .
$$

Remark 5.1.5. In view of Proposition 5.1.3, $\mathcal{E}_{\{\omega\}}^{t}(\Omega, E) \subset \mathcal{E}_{\{\omega\}}(\Omega, E)$.

The Definitions 5.1.1 and 5.1.4 should be compared with the notions of $C^{M}$-curve or strongly $C^{M}$-curve in [44, Definition 3.1] given by Kriegl, Michor and Rainer. These authors consider increasing weight sequences $M=\left(M_{p}\right)_{p \in \mathbb{N}_{0}}$ satisfying (M1) and (M2') as in Definition 1.0.18 and define 
Vector valued ultradifferentiable functions of Roumieu type in Fréchet 80

spaces

ultradifferentiable curves with values in a locally convex space, $c: \mathbb{R} \rightarrow E$, using the approach of Carleman and Komatsu [38] (see also Definition 1.0.19). A curve $c: \mathbb{R} \rightarrow E$ is called $\mathcal{C}^{M}$ if for all $u \in E^{\prime}, u \circ c \in \mathcal{E}_{\left\{M_{p}\right\}}(\mathbb{R})$. The curve $c$ is called strongly $\mathcal{C}^{M}$ if for all compact subset $K \subset \mathbb{R}$ there is $h>0$ such that the set

$$
\left\{\frac{c^{(p)}(x)}{h^{p} M_{p}}: \quad x \in K, \quad p \in \mathbb{N}_{0}\right\}
$$

is bounded in $E$.

We introduce now bornologically $\omega$-ultradifferentiable functions. First, we recall the notion of Banach Disk. Let $B$ be an absolutely convex bounded subset in $E$. Denote by $\|\cdot\|_{B}$ the Minkowski functional of $B$, that is, for each $f \in E$

$$
\|f\|_{B}=\inf \{t>0: \quad f \in t B\} .
$$

We set $E_{B}=\operatorname{span} E=\bigcup_{t} t B . E_{B}$ is a normed space endowed with the Minkowski functional of $B$. In case $E$ is sequentially complete one can prove that $E_{B}$ is a Banach space and the inclusion $E_{B} \hookrightarrow E$ is always contiunuous.

An absolutely convex bounded subset $B$ in a locally convex space $E$ is called Banach disk if the space $E_{B}$ is Banach.

Definition 5.1.6. A function $f: \Omega \rightarrow E$ is bornologically $\omega$-ultradifferentiable (of Roumieu type), and we denote $f \in \mathcal{E}_{\{\omega\}}^{b}(\Omega, E)$, if there exists a closed absolutely convex bounded subset $B$ (a Banach disk since $E$ is sequentially complete) such that

$$
f \in \mathcal{E}_{\{\omega\}}\left(\Omega, E_{B}\right) .
$$

Our purpose is to study the equalities

$$
\mathcal{E}_{\{\omega\}}^{t}(\Omega, E)=\mathcal{E}_{\{\omega\}}(\Omega, E) \text { and } \mathcal{E}_{\{\omega\}}^{t}(\Omega, E)=\mathcal{E}_{\{\omega\}}^{b}(\Omega, E)
$$

when $E$ is a Fréchet or an (LB)-space, thus answering a question by Kriegl and Michor. To do this, following an idea of Bonet and Domanski [9], we must represent $\mathcal{E}_{\{\omega\}}(\Omega, E)$ as a tensor product. Recall that given locally convex spaces $X$ and $Y$, their Schwartz's e-product is $X \varepsilon Y:=L_{e}\left(X_{c o}^{\prime}, Y\right)$. It is endowed with the topology of uniform convergence on equicontinuous sets in $X^{\prime}$. Here $X_{c o}^{\prime}$ is the topological dual of $X$ with the topology of uniform convergence on convex compact subsets in $X$ and $X_{\beta}^{\prime}$ the topological dual with the topology of uniform convergence on bounded subsets. See [41, II]. Of course, if $X$ is Montel then $X \varepsilon Y=L_{\beta}\left(X_{\beta}^{\prime}, Y\right)$. A linear map 
$f: X \rightarrow Y$ is called bounded if there is a 0 -neighborhood $U$ in $X$ such that $f(U)$ is bounded in $Y$, or equivalently, there is a bounded disk $B$ in $Y$ such that $f: X \rightarrow Y_{B}$ is continuous. In this case, we write $f \in L B(X, Y)$.

Lemma 5.1.7. The linear $H$ span of $\left\{\delta_{x}: x \in \Omega\right\}$ is a sequentially dense subset of $\mathcal{E}_{\{\omega\}}(\Omega)_{\beta}^{\prime}$.

Proof. Fix $u \in \mathcal{E}_{\{\omega\}}(\Omega)_{\beta}^{\prime}$. There is a compact subset $K$ such that $u \in \mathcal{E}_{\{\omega\}}(K)^{\prime}$. The $(L B)$-space $\mathcal{E}_{\{\omega\}}(K)$ is reflexive and if $f \in \mathcal{E}_{\{\omega\}}(K)$ verifies $\left\langle\delta_{x}, f\right\rangle=0$ for all $x \in K$, then $f=0$. A Hahn-Banach argument implies that $H$ is weak-star dense in the Fréchet space $\mathcal{E}_{\{\omega\}}(K)_{\beta}^{\prime}$. Therefore, there exists a sequence $\left\{u_{j}\right\}_{j \in \mathbb{N}_{0}} \subset H$ such that $u_{j}$ tends to $u$ in $\mathcal{E}_{\{\omega\}}(K)_{\beta}^{\prime}$ and hence, $u_{j}$ tends to $u$ in $\mathcal{E}_{\{\omega\}}(\Omega)_{\beta}^{\prime}$.

Theorem 5.1.8. Let E be a sequentially complete locally convex space. The spaces $\mathcal{E}_{\{\omega\}}(\Omega, E)$ and $L\left(\mathcal{E}_{\{\omega\}}(\Omega)_{\beta}^{\prime}, E\right)$ are algebraically isomorphic. Moreover, this isomorphism maps $\mathcal{E}_{\{\omega\}}^{b}(\Omega, E)$ onto $\operatorname{LB}\left(\mathcal{E}_{\{\omega\}}(\Omega)_{\beta}^{\prime}, E\right)$.

Proof. We define $\Delta: \Omega \rightarrow \mathcal{E}_{\{\omega\}}(\Omega)_{\beta}^{\prime}$ by $\Delta(x)=\delta_{x}$. The map

$$
\Phi: L\left(\mathcal{E}_{\{\omega\}}(\Omega)_{\beta}^{\prime}, E\right) \rightarrow \mathcal{E}_{\{\omega\}}(\Omega, E), \Phi(W)=W \circ \Delta,
$$

is well defined and linear. In fact, for every $u \in E^{\prime}$ we have $u \circ W \in$ $\mathcal{E}_{\{\omega\}}(\Omega)^{\prime \prime}$, hence, by reflexivity, there is $\varphi \in \mathcal{E}_{\{\omega\}}(\Omega)$ such that

$$
\varphi(x)=\delta_{x}(\varphi)=(u \circ W)\left(\delta_{x}\right)=u(\Phi(W))(x),
$$

which proves that $u \circ \Phi(W)=\varphi \in \mathcal{E}_{\{\omega\}}(\Omega)$. Now we define $H:=$ $\operatorname{span}\left\{\delta_{x}: x \in \Omega\right\}$ and we endow it with the topology induced by $\mathcal{E}_{\{\omega\}}(\Omega)_{\beta}^{\prime}$. If $f \in \mathcal{E}_{\{\omega\}}(\Omega, E)$ we denote by $\Psi(f): H \rightarrow E$ the linear extension of the map $\Psi(f)\left(\delta_{x}\right):=f(x), x \in \Omega$. We check that $\Psi(f)$ is continuous. Fix a continuous seminorm $q$ on $E$ and consider the bounded set $B_{q}$ in $\mathcal{E}_{\{\omega\}}(\Omega)$ defined by $B_{q}:=\left\{u \circ f:|u| \leq q, u \in E^{\prime}\right\}$. If $y \in H$ belongs to $B_{q}^{\circ}$ then

$$
q(\Psi(f)(y))=\sup _{|u| \leq q}|u(\Psi(f)(y))| \leq 1
$$

By Lemma 5.1.7, $H$ is sequentially dense in $\mathcal{E}_{\{\omega\}}(\Omega)_{\beta}^{\prime}$ and there is a unique continuous and linear extension $\Psi(f): \mathcal{E}_{\{\omega\}}(\Omega)_{\beta}^{\prime} \rightarrow E$. That is, $\Psi(f) \in$ $L\left(\mathcal{E}_{\{\omega\}}(\Omega)_{\beta}^{\prime}, E\right)$ and the map

$$
\Psi: \mathcal{E}_{\{\omega\}}(\Omega, E) \rightarrow L\left(\mathcal{E}_{\{\omega\}}(\Omega)_{\beta}^{\prime}, E\right)
$$


Vector valued ultradifferentiable functions of Roumieu type in Fréchet 82

spaces

is well defined and linear. Since $\Phi \circ \Psi$ and $\Psi \circ \Phi$ coincide with the identity, both $\Phi$ and $\Psi$ are linear isomorphisms. Finally we see that the isomorphism $\Psi$ maps $\mathcal{E}_{\{\omega\}}^{b}(\Omega, E)$ onto $L B\left(\mathcal{E}_{\{\omega\}}(\Omega)_{\beta}^{\prime}, E\right)$. Let $f \in \mathcal{E}_{\{\omega\}}^{b}(\Omega, E)$ be given. There is a Banach disk $B$ such that $f \in \mathcal{E}_{\{\omega\}}\left(\Omega, E_{B}\right)$ and

$$
\Psi(f) \in L\left(\mathcal{E}_{\{\omega\}}(\Omega)_{\beta^{\prime}}^{\prime} E_{B}\right) \subseteq L B\left(\mathcal{E}_{\{\omega\}}(\Omega)_{\beta}^{\prime}, E\right) .
$$

Conversely, if $W \in \operatorname{LB}\left(\mathcal{E}_{\{\omega\}}(\Omega)_{\beta}^{\prime}, E\right)$ there is a Banach disk $B$ such that $W \in L\left(\mathcal{E}_{\{\omega\}}(\mathbb{R})_{\beta}^{\prime}, E_{B}\right)$. Then, $\Phi(W) \in \mathcal{E}_{\{\omega\}}\left(\mathbb{R}, E_{B}\right) \subseteq \mathcal{E}_{\{\omega\}}^{b}(\mathbb{R}, E)$.

Lemma 5.1.9. Let $E$ be a Fréchet space If $f \in \mathcal{C}^{\infty}(\Omega, E)$, then there is a closed Banach disc $B$ in $E$ such that $f \in \mathcal{C}^{\infty}\left(\Omega, E_{B}\right)$.

Proof. Let $\left\{K_{n}\right\}_{n \in \mathbb{N}}$ be a compact exhaustion of $\Omega$ and, for each $n \in \mathbb{N}$, we consider the compact set

$$
B_{n}:=\bigcup\left\{f^{(\alpha)}\left(K_{n}\right):|\alpha| \leq n\right\}
$$

According to a result due to Mackey (see [41, I, 29.1 (5)]) there are $\lambda_{n}>0$ such that $C:=\bigcup_{n \in \mathbb{N}} \lambda_{n} B_{n}$ is a bounded set in $E$. We denote by $B$ the closure of the absolute convex hull of $C$ and prove that $f \in \mathcal{C}^{\infty}\left(\Omega, E_{B}\right)$. It is easy to see that $f \in \mathcal{C}\left(\Omega, E_{B}\right)$. Proceeding by induction, let us assume that $f \in \mathcal{C}^{m}\left(\Omega, E_{B}\right)$ and fix a compact subset $K \subset \Omega$, a multi-index $\alpha$ with $|\alpha|=m$ and $1 \leq j \leq N$. Take $n \in \mathbb{N}$ such that $K$ is contained in the interior of $K_{n}$ and find $\mu>0$ with $f^{(\beta)}\left(K_{n}\right) \subset \mu B$ whenever $|\beta| \leq m+2$. Then, for every $x \in K, u \in B^{\circ}$ and $t \neq 0$ small enough we have, after applying Taylor' Theorem to the real-valued function $u \circ f$,

$$
\left\langle\frac{f^{(\alpha)}\left(x+t e_{j}\right)-f^{(\alpha)}(x)}{t}-\frac{\partial}{\partial x_{j}} f^{(\alpha)}(x), u\right\rangle=\frac{t}{2}\left\langle\frac{\partial^{2}}{\partial x_{j}^{2}} f^{(\alpha)}\left(x+\xi e_{j}\right), u\right\rangle
$$

for some $\xi$ such that $|\xi| \leq|t|$. Hence

$$
\sup _{x \in K}\left\|\frac{f^{(\alpha)}\left(x+t e_{j}\right)-f^{(\alpha)}(x)}{t}-\frac{\partial}{\partial x_{j}} f^{(\alpha)}(x)\right\|_{E_{B}} \leq \mu|t|,
$$

which proves that $f \in \mathcal{C}^{m+1}\left(\Omega, E_{B}\right)$.

Theorem 5.1.10. If $E$ is a Fréchet space, then, $\mathcal{E}_{\{\omega\}}^{t}(\Omega, E)=\mathcal{E}_{\{\omega\}}^{b}(\Omega, E)$. 
Proof. Let $f \in \mathcal{E}_{\{\omega\}}^{b}(\Omega, E)$ be given. By the previous lemma there is a Banach disk $B$ such that $f \in \mathcal{E}_{\{\omega\}}\left(\Omega, E_{B}\right)$. Moreover, according to Proposition 5.1.3, for all compact subset $K$ there is $m$ such that

$$
\sup _{x \in K} \sup _{\alpha \in \mathbb{N}_{0}^{N}}\left\|f^{(\alpha)}(x)\right\|_{E_{B}} \exp \left(-\frac{1}{m} \varphi^{*}(|\alpha| m)\right)<\infty .
$$

Since the inclusion $E_{B} \hookrightarrow E$ is continuous it easily follows that $f \in \mathcal{E}_{\{\omega\}}^{t}(\Omega, E)$.

Conversely, let us assume that $f \in \mathcal{E}_{\{\omega\}}^{t}(\Omega, E)$ and fix a compact exhaustion $\left\{K_{n}\right\}_{n \in \mathbb{N}}$ of $\Omega$. For each $n$ there is $m_{n} \in \mathbb{N}$ such that the set

$$
B_{n}:=\left\{f^{(\alpha)}(x) \exp \left(-\frac{1}{m_{n}} \varphi^{*}\left(|\alpha| m_{n}\right)\right): x \in K_{n}, \alpha \in \mathbb{N}_{0}^{N}\right\}
$$

is bounded in $E$. From (the proof of) Lemma 5.1.9 there are $\lambda_{n}>0$ such that the closed absolutely convex hull $B$ of $\bigcup_{n \in \mathbb{N}} \lambda_{n} B_{n}$ is a Banach disc and $f \in \mathcal{C}^{\infty}\left(\Omega, E_{B}\right)$. Moreover, from the definition of $B_{n}$ we have that

$$
\sup _{x \in K_{n}} \sup _{\alpha \in \mathbb{N}_{0}^{N}}\left\|f^{(\alpha)}\right\|_{E_{B}} \exp \left(-\frac{1}{m_{n}} \varphi^{*}\left(|\alpha| m_{n}\right)\right) \leq \lambda_{n}^{-1},
$$

from where we conclude that $f \in \mathcal{E}_{\{\omega\}}^{b}(\Omega, E)$.

The next theorem is proved in [44, Lemma 5.2] for functions $f: \mathbb{R}^{\mathbb{N}} \rightarrow$ $E$ in the setting of Denjoy-Carlemann classes with a different proof.

Theorem 5.1.11. Let $E$ be a sequentially complete locally convex space such that $E_{\beta}^{\prime}$ is a Baire space. Then, $\mathcal{E}_{\{\omega\}}(\Omega, E)=\mathcal{E}_{\{\omega\}}^{t}(\Omega, E)$.

Proof. In view of Proposition 5.1.3 it is clear that $\mathcal{E}_{\{\omega\}}^{t}(\Omega, E) \subseteq \mathcal{E}_{\{\omega\}}(\Omega, E)$. In order to show the other inclusion, let $f \in \mathcal{E}_{\{\omega\}}(\Omega, E)$ fixed and $K$ compact subset. The map $T: E_{\beta}^{\prime} \rightarrow \mathcal{E}_{\{\omega\}}(K)$ given by $T(u)=\left.(u \circ f)\right|_{K}$ has closed graph and we can apply a Grothendieck's Factorization Theorem $\left(\left[58,1.2 .20\right.\right.$ (i)]) to conclude that there is $m \in \mathbb{N}$ such that $T: E_{\beta}^{\prime} \rightarrow \mathcal{E}_{\{\omega\}}^{\frac{1}{m}}(K)$ is well defined and continuous. Therefore, the set

$$
\left\{f^{(\alpha)}(x) \exp \left(-\frac{1}{m} \varphi^{*}(|\alpha| m)\right): x \in K, \alpha \in \mathbb{N}_{0}^{N}\right\}
$$

is weakly bounded in $E$, hence it is bounded. 
Vector valued ultradifferentiable functions of Roumieu type in Fréchet 84

spaces

\subsection{Main results}

Let $\omega$ be a non-quasianalytic weight function. We characterize when the three definitions of vector valued $\{\omega\}$-ultradifferentiable function given in the previous section coincide if $E$ is a Fréchet space or a complete LBspace. We start with the following example due to Kriegl, Michor and Rainer [44].

Example 5.2.1. Let $E$ be the countable product of copies of the scalar field, endowed with the product topology, which is a nuclear Fréchet space. Let $\omega(t)=t^{1 / \alpha}, s:=1 / \alpha>1$, be a Gevrey weight. There are curves in $\mathcal{E}_{\{\omega\}}(\mathbb{R}, E)$ which are not in $\mathcal{E}_{\{\omega\}}^{t}(\mathbb{R}, E)$ by [44, Example 3.2]. This fact is based on the existence of $f \in \mathcal{E}_{\{\omega\}}(\mathbb{R})$ such that

$$
\left\{f^{(\alpha)}(x) \exp \left(-\lambda \varphi^{*}\left(\frac{|\alpha|}{\lambda}\right)\right): \alpha \in \mathbb{N}_{0}, x \in K\right\}
$$

is unbounded for any compact subset $K \subset \mathbb{R}$ containing 0 and a suitable $\lambda$.

We recall the definition of topological invariant (DN) of Vogt [54].

Definition 5.2.2. A Fréchet space $E$ with a fundamental system of seminorms $\left(\|\cdot\|_{n}\right)_{n \in \mathbb{N}}$ is said to satisfy the property (DN) of Vogt if

$$
\exists n \forall m \geq n \exists l \geq m, C<\infty \forall x \in E:\|x\|_{m}^{2} \leq C\|x\|_{n}\|x\|_{l} .
$$

In fact, $\|\cdot\|_{n}$ is a norm which is called dominating norm. Every power series spaces on infinite type has the property (DN) and a nuclear Fréchet space has the property (DN) if and only if it is isomorphic to a subspace of the space $s$ of rapidly decreasing sequences. See [54, Chapter 29] for more details.

Power series spaces of order one are defined as follows. Let $\alpha=$ $\left(\alpha_{j}\right)_{j \in \mathbb{N}}$ be a monotonically increasing sequence in $\left[0, \infty\left[\right.\right.$ with $\lim _{j} \alpha_{j}=\infty$, we set

$$
\Lambda_{1}(\alpha)=\left\{x \in \mathbb{C}^{N}:\|x\|_{r}:=\sum_{j \in \mathbb{N}}\left|x_{j}\right| r^{\alpha_{j}}<\infty \text { for all } 0<r<1\right\} .
$$

$\Lambda_{1}(\alpha)$ is a reflexive Fréchet space equipped with the fundamental system of norms

$$
\left\{\|\cdot\|_{r}\right\}_{0<r<1} \cdot
$$

In view of [63, Theorem 2.1], for positive increasing sequences $\alpha=\left(\alpha_{j}\right)$ verifying $\sup _{j} \frac{\alpha_{j+1}}{\alpha_{j}}<\infty$, a Fréchet space $E$ has (DN) property if and only if $L\left(\Lambda_{1}(\alpha), E\right)=L B\left(\Lambda_{1}(\alpha), E\right)$. The next theorem is a consequence of [22, Proposition 4.9]. 
Theorem 5.2.3. Let $\omega$ be a non-quasianalytic weight, $\Omega$ an open subset in $\mathbb{R}^{N}$ and $\alpha(\omega, N):=\left\{\omega\left(j^{\frac{1}{N}}\right)\right\}_{j \in \mathbb{N}}$. Then,

$$
\mathcal{E}_{\{\omega\}}(\Omega)^{\prime}=\bigoplus_{\mathbb{N}} \Lambda_{1}(\alpha(\omega, N)) .
$$

Theorem 5.2.4. Let $\omega$ be a non-quasianalytic weight and let $E$ be a Fréchet space. Then $\mathcal{E}_{\{\omega\}}(\Omega, E)=\mathcal{E}_{\{\omega\}}^{t}(\Omega, E)$ if and only if $E$ has the property $(D N)$.

Proof. We first suppose $\mathcal{E}_{\{\omega\}}(\Omega, E)=\mathcal{E}_{\{\omega\}}^{t}(\Omega, E)$. In view of Theorems 5.1.8 and 5.1.10 the identity

$$
L\left(\mathcal{E}_{\{\omega\}}(\Omega)_{\beta}^{\prime}, E\right)=L B\left(\mathcal{E}_{\{\omega\}}(\Omega)_{\beta}^{\prime}, E\right)
$$

holds and from the fact that $\Lambda_{1}\left(\left\{\omega\left(j^{\frac{1}{N}}\right)\right\}_{j \in \mathbb{N}}\right)$ is a complemented subspace of $\mathcal{E}_{\{\omega\}}(\Omega)^{\prime}$ it easily follows that also

$$
L\left(\Lambda_{1}\left(\left\{\omega\left(j^{\frac{1}{N}}\right)\right\}_{j \in \mathbb{N}}\right), E\right)=L B\left(\Lambda_{1}\left(\left\{\omega\left(j^{\frac{1}{N}}\right)\right\}_{j \in \mathbb{N}}\right), E\right) .
$$

On the other hand, condition $(\alpha)$ of weight function implies $\sup _{j} \frac{\omega\left((j+1)^{\frac{1}{N}}\right)}{\omega\left(j \frac{1}{N}\right)}<$ $\infty$. Hence we can apply [63, Theorem 2.1] to conclude that $E$ satisfies (DN).

Conversely, suppose that $E$ has the (DN) property. Then,

$$
L\left(\Lambda_{1}\left(\left\{\omega\left(j^{\frac{1}{N}}\right)\right\}_{j \in \mathbb{N}}\right), E\right)=L B\left(\Lambda_{1}\left(\left\{\omega\left(j^{\frac{1}{N}}\right)\right\}_{j \in \mathbb{N}}\right), E\right) .
$$

Now, it is enough to apply Theorem 5.2.3, [54, Proposition 24.3] and [1, Proposition 1] to get that also $L\left(\mathcal{E}_{\{\omega\}}(\Omega)_{\beta}^{\prime}, E\right)=L B\left(\mathcal{E}_{\{\omega\}}(\Omega)_{\beta}^{\prime}, E\right)$. Hence, Theorem 5.1.8 permits to conclude that $\mathcal{E}_{\{\omega\}}(\Omega, E)=\mathcal{E}_{\{\omega\}}^{t}(\Omega, E)$.

Bonet, Meise and Melikhov characterize in [17, Theorem 14] those weight sequences $\left(M_{p}\right)_{p \in \mathbb{N}_{0}}$ for which there exits a weight function $\omega$ such that the corresponding class of ultradifferentiable functions defined by $\left(M_{p}\right)_{p \in \mathbb{N}_{0}}$ coincide with the class of ultradifferentiable functions in the sense of Braun, Meise and Taylor defined by $\omega$. To do this, these authors introduced the following conditions on the weight sequence $\left(M_{p}\right)_{p \in \mathbb{N}_{0}}$.

We say that $\left(M_{p}\right)_{p \in \mathbb{N}_{0}}$ verifies (M2) if it has the following property stronger than (M2)':

there are $A, H>1$ such that $M_{p} \leq A H^{p} \min _{0 \leq p \leq q} M_{p} M_{p-q}, \quad p \in \mathbb{N}_{0}$.

We set $m_{p}:=\frac{M_{p}}{M_{p-1}}$. We say that $\left(M_{p}\right)_{p \in \mathbb{N}_{0}}$ verifies B-M-M* if there exists $Q \in \mathbb{N}$ with $\lim \inf _{j \rightarrow \infty} \frac{m_{Q j}}{m_{j}}>1$. 
Vector valued ultradifferentiable functions of Roumieu type in Fréchet 86

spaces

Corollary 5.2.5. Let $\left(M_{p}\right)_{p \in \mathbb{N}_{0}}$ be a sequence of positive numbers satisfying (M0), (M1), (M2), (M3) and condition B-M-M*. A Fréchet space E satisfies $(D N)$ if and only if every $C^{M}$ curve with values in $E$ is a strong $C^{M}$ curve.

Proof. By [17, Theorem 14], $C^{M}$ curves in E correspond to elements in $\mathcal{E}_{\{\omega\}}(] a, b[, E)$ and strong $C^{M}$ curves in $E$ correspond to elements in $\mathcal{E}_{\{\omega\}}^{t}(] a, b[, E)$. The conclusion follows by Theorem 5.2.4.

Gevrey classes satisfy all the assumptions of Corollary 3.5.

In the real analytic case, the property which characterizes those Fréchet spaces $E$ for which $\mathcal{A}(\Omega, E)=\mathcal{A}_{t}(\Omega, E)$ holds is the condition (DN) (see [9, Theorem 18] and [10, Theorem 3]). The property which characterizes those (LB)-spaces for which $\mathcal{A}(\Omega, E)=\mathcal{A}_{b}(\Omega, E)$ holds is that $E_{\beta}^{\prime}$ has the property $(\overline{\bar{\Omega}})$ of Vogt (see [9, Theorem 21] and [10, Theorem 5]). However, in the ultradifferentiable setting we get the following result.

Theorem 5.2.6. Let $\omega$ be a non-quasianalytic weight and let $E=\operatorname{ind}_{n} E_{n}$ be a complete LB-space. Then $\mathcal{E}_{\{\omega\}}(\Omega, E)=\mathcal{E}_{\{\omega\}}^{b}(\Omega, E)$ if and only if $E$ is a Banach space.

Proof. Let us assume that $\mathcal{E}_{\{\omega\}}(\Omega, E)=\mathcal{E}_{\{\omega\}}^{b}(\Omega, E)$ and $E$ is not normable. From Theorems 5.1.8 and 5.2.3 we get

$$
L\left(\bigoplus_{\mathbb{N}} \Lambda_{1}(\alpha(\omega, N)), E\right)=L B\left(\bigoplus_{\mathbb{N}} \Lambda_{1}(\alpha(\omega, N)), E\right) .
$$

Let $\varphi$ denote the countable direct sum of copies of the scalar field. We take $x \in \Lambda_{1}(\alpha(\omega, N))$ and $u \in \Lambda_{1}(\alpha(\omega, N))^{\prime}$ such that $u(x)=1$, define $\pi: \oplus_{\mathbb{N}} \Lambda_{1}(\alpha(\omega, N)) \rightarrow \varphi$ and $i: \varphi \rightarrow \oplus_{\mathbb{N}} \Lambda_{1}(\alpha(\omega, N))$ by $\pi\left(\left\{z_{n}\right\}_{n}\right)=$ $\left\{u\left(z_{n}\right)\right\}_{n}$ and $i\left(\left\{\alpha_{n}\right\}_{n}\right)=\left\{\alpha_{n} x\right\}$. It is clear that $\pi \circ i=I d_{\varphi}$, hence $\varphi$ is a complemented subspace of $\bigoplus_{\mathbb{N}} \Lambda_{1}(\alpha(\omega, N)$. It follows that $L(\varphi, E)=$ $\operatorname{LB}(\varphi, E)$. This is a contradiction. Indeed, since $E$ is not normable we can find a subsequence $\left(n_{k}\right)_{k} \subset \mathbb{N}$ and $x_{k} \in E_{n_{k+1}} \backslash E_{x_{k}}$. We can apply a result by Bonet ([7, Proposition 11]) to find $\left.\left(C_{k}\right)_{k \in \mathbb{N}} \subset\right] 0 . \infty\left[\right.$ such that $\left(C_{k} x_{k}\right)_{k \in \mathbb{N}}$ is bounded in $E$. This is impossible because every complete LB-space is regular and $\left(C_{k} x_{k}\right)_{k \in \mathbb{N}}$ is not included in any step. 


\section{Bibliography}

[1] A. A. Albanesse; Bounded linear maps between (LF)-spaces, RACSAM 97(1) (2003), 13-31.

[2] A. Beurling; Quasianalyticity and general distributions. Lecture 4 and 5, AMS Summer Institute. Standford (1961).

[3] G. Björck; Linear partial differential operators and generalized distributions, Ark. Mat. 6 (1965), 351-407.

[4] P. Bolley, J. Camus; Regularité Gevrey et itérés pour une classe d'opérateurs hypoelliptiques, Comm. Partial Differential Equations 10(6) (1981), 1057-1110.

[5] P. Bolley, J. Camus, C. Mattera; Analyticité microlocale et itérés d'opérateurs, Séminaire Goulaouic-Schwartz (1978/1979), Exp. No. 13, 9 pp., École Polytech., Palaiseau.

[6] P. Bolley, J. Camus, L. Rodino; Analytic Gevrey hypoellipticity and iterates of operators, Rend. Sem. Mat. Univ. Politec. Torino 45(3) (1987), $1-61$.

[7] J. Bonet; On the identity $L(E, F)=L B(E, F)$ for pairs of locally convex spaces $E$ and F, Proc. Amer. Math. Soc. 99(2) (1987), 249-255.

[8] J. Bonet; El impacto del análisis funcional en algunos problemas del análisis. Discurso leído en el acto de su recepción como académico de número por el Excmo. Sr. D. José Bonet Solves y contestación del Excmo. Sr. D. Manuel Valdivia Ureña. Madrid, 23 de abril de 2008. ISBN 978-848363-253-6.

[9] J. Bonet, P. Domański; Real analytic curves in Fréchet spaces and their duals, Mh. Math. 126 (1998), 13-36.

[10] J. Bonet, P. Domański; Parameter dependence of the solutions partial differential operators in spaces of real analytic functions, Proc. Amer. Math. Soc. 129(2) (2001), 495-503. 
[11] J. Bonet, P. Domański; Parameter dependence of solutions of differential equations on spaces of distributions and the splitting of short exact sequences, J. Funct. Anal. 230(2) (2006), 329-381.

[12] J. Bonet, P. Domański; The splitting of exact sequences of PLS-spaces and smooth dependence of solutions of linear partial differential equations, Adv. Math. 217 (2008), 561-585.

[13] J. Bonet, C. Fernández and R. Meise; Operators of solution for convolution equations, Note Mat. 17 (1997), 1-12.

[14] J. Bonet, C. Fernández, R. Meise; Characterization of the w-hypoelliptic convolution operators on ultradistributions, Ann. Acad. Sci. Fenn. Math. 25 (2000), 261-284.

[15] J. Bonet, A. Galbis and R. Meise; On the range of convolution operators on non-quasianalytic ultradifferentiable functions, Studia Math. 126 (1997), 171-198.

[16] J. Bonet, A. Galbis, S. Momm; Nonradial Hörmander algebras of several variables and convolution operators, Trans. Amer. Math. Soc. 353(6) (2001), 2275-2291.

[17] J. Bonet, R. Meise, S.N.Melikhov; A comparison of two different ways of define classes of ultradifferentiable functions, Bull. Belg. Math. Soc. Simon Stevin 14 (2007), 425-444.

[18] C. Bouzar, R. Chaili; Une généralisation du problème des itérés, Arch. Math. (Basel) 76 (1) (2001), 57-66.

[19] C. Bouzar, R. Chaili; Gevrey vectors of multi-quasi-elliptic systems, Proc. Amer. Math. Soc. 5 (131) (2003), 1565-1572.

[20] C. Bouzar, L. Chaili; A Gevrey microlocal analysis of multi-anisotropic differential operators, Rend. Semin. Mat. Univ. Politec. Torino 64(3) (2006), 305-317.

[21] R.W. Braun; An extension of Komatsu's second structure theorem for ultradistributions, J. Fac. Sci. Univ. Tokyo, Sect. IA Math. 40 (1993), 411-417.

[22] R.W. Braun, R. Meise, B.A. Taylor; Ultradifferentiable functions and Fourier analysis, Result. Math. 17 (1990), 206-237.

[23] D. Calvo, G. H. Hakobyan; Multi-anisotropic Gevrey regularity and iterates of operators with constant coefficients, Bull. Belg. Math. Soc. Simon Stevin 12 (3) (2005), 461-474. 
[24] D. Calvo, L. Rodino; Iterates of operators and Gelfand-Shilov classes, to appear in Integral Transforms and Special Functions.

[25] C. Fernández, A. Galbis, M.C. Gómez-Collado; Elliptic convolution operators on non-quasianalytic classes, Arch. Math. 76 (2001), 133-140.

[26] C. Fernández, A. Galbis, D. Jornet; $\omega$-hypoelliptic differential operators of constant strength, J. Math. Anal. Appl. 297 (2004), 561-576.

[27] C. Fernández, A. Galbis, D. Jornet; Pseudodifferential operators on nonquasianalytic classes of Beurling type, Studia Math. 167(2) (2005), 99-131.

[28] C. Fernández, A. Galbis, J. Juan-Huguet; Fréchet spaces invariant under differential operators. Accepted in Functiones et Approximatio, Commentarii Mathematici.

[29] L. Frerick, J. Wengenroth; Convolution equations for ultradifferentiable functions and ultradistributions, J. Math. Anal. Appl. 297 (2004), 506517.

[30] M. Gevrey; Sur la nature analytique des solutions des équations aux dérivées partielles. Premier mémoire, Ann. Sci. École Norm. Sup. (3) 35 (1918), 129-190.

[31] A. Grothendieck; Sur certains espaces de fonctions holomorphes. I, II, J. reine angew. Math. 192 (1953), 35-64, 77-95.

[32] L. Hörmander; On interior regularity of the solutions of partial differential equations, Comm. Pure Appl. Math. 11(1958), 197-218.

[33] L. Hörmander; The Analysis of Linear Partial Differential Operators I and II. Springer-Verlag, Berlin (1990).

[34] H. Jarchow; Locally Convex Spaces. Teubner, Sttuttgart (1981).

[35] J. Juan-Hughet; Iterates and hypoellipticity of differential operators on non quasi analytic classes, Integr. Equ. Oper. Theory 68(2) (2010), 263-286.

[36] J. Juan-Huguet; Non quasi analytic curves in Fréchet spaces. Accepted in Monatshefte für Mathematik.

[37] H. Komatsu; A characterization of real analytic functions, Proc. Japan Acad. 36(1960), 90-93.

[38] H. Komatsu; Ultradistributions I. Structure theorems and a characterization, J. Fac. Sci. Tokyo Sec.IA Math 20 (1973), 25-105. 
[39] H. Komatsu; Ultradistributions III. Vector valued ultradistributions and the theory of kernels, J. Fac. Sci. Tokyo Sec.IA Math 29 (1982) 653-717.

[40] T. Kotake, M. Narasimhan; Regularity theorems for fractional powers of a linear elliptic operator, Bull. Soc. Math. France 90 (1962),449-471.

[41] G. Köthe; Topological Vector Spaces I and II. Springer-Verlag, Berlin $(1969,1979)$.

[42] A. Kriegl, P. W. Michor; The convenient setting for real analytic mappings, Acta Math. 165 (1990), 105-159.

[43] A. Kriegl, P.W. Michor; The convenient setting of global analysis. Mathematical Surveys and Monographs 53. American Mathematical Society, Providence, RI, (1997).

[44] A. Kriegl, P. W. Michor, A. Rainer; The convenient setting for nonquasianalytic Denjoy- Carleman differentiable mappings, J. Funct. Anal. 256 (2009), 3510-3544.

[45] M. Langenbruch; P-Funktionale und Randwerte zu hypoelliptischen Differentialoperatoren, Math. Ann. 239(1) (1979), 55-74.

[46] M. Langenbruch; Fortsetzung von Randwerten zu hypoelliptischen Differentialoperatoren und partielle Differentialgleichungen, J. reine angew. Math. 311/312 (1979), 57-79.

[47] M. Langenbruch; On the functional dimension of solution spaces of hypoelliptic partial differential operators, Math. Ann. 272 (1985), 217-229.

[48] M. Langenbruch; Bases in solution sheaves of systems of partial differential equations, J. reine angew. Math. 373 (1987), 1-36.

[49] M. Langenbruch; Continuation of Gevrey regularity for solutions of partial differential operators, In: "Functional Analysis" (Proceedings of the First International Workshop held at Trier University 1994), Walter de Gruyter (1996), 249-280.

[50] M. Langenbruch, J. Voigt; On Banach spaces invariant under differentiation, Bull. Soc. Roy. Sci. Liège 69 (2000), 387-393.

[51] B. M. Makarov; Some pathological properties of inductive limits of Bspaces, Uspehi Mat. Nauk 18(3) (1963), 171-178.

[52] R. Meise, B.A. Taylor; Whitney's extension theorem for ultradifferentiable functions of Beurling type, Ark. Mat. 26 (1988), 265-287. 
[53] R. Meise, B.A. Taylor, D. Vogt; Continuous linear right inverses for partial differential operators on non-quasianalytic classes and ultradistributions, Math. Nachr. 180 (1996), 213-242.

[54] R. Meise, D. Vogt; Introduction to Functional Analysis. Clarendon Press, Oxford (1997).

[55] G. Métivier; Propiété des itérés et ellipticité, Comm. Partial Differential Equations 39 (1978), 827-876.

[56] J. Mujica; A completeness criterion for inductive limits of Banach spaces, Functional Analysis, Holomorphy and Approximation Theory II (ed.: G.I. Zapata). North-Holland Math Studies (1984).

[57] E. Newberger, Z. Zielezny; The growth of hypoelliptic polynomials and Gevrey clases, Proc. Amer. Math. Soc. 39(3) (1973), 547-552.

[58] P. Pérez-Carreras, J. Bonet; Barrelled Locally Convex Spaces. NothHolland Mathematics Studies 131 (1987).

[59] W. Rudin. Real and Complex Analysis (Third Edition). WCB/McGrawHill Book Company, New York (1973).

[60] L. Schwartz; Théorie des distributions à valeurs vectorielles I, Ann. Inst. Fourier 7 (1957), 1-141.

[61] L. Schwartz; Théorie des distributions à valeurs vectorielles II, Ann. Inst. Fourier 8 (1958), 1-209.

[62] E.M. Stein, G.Weiss. Introduction to Fourier Analysis on Euclidean Spaces. Princeton University Press. (1971)

[63] D. Vogt. Frécheträume, zwischen denen jede stetige lineare Abbildung beschränkt ist, J. reine angew. Math 345 (1983), 182-200.

[64] J. Wengenroth; Derived Functors in Funcional Analysis. SpringerVerlag. Lecture Notes in Mathematics 1810, Berlin (2003).

[65] L.Zanghirati; Iterates of a class of hypoelliptic operators and generalized Gevrey classes, Boll. Un. Mat. Ital. Suppl. 1 (1980), 177-195.

[66] L. Zanghirati; Iterates of quasielliptic operators and Gevrey classes, Boll. U.M.I. 18-B (1981), 411-428.

[67] L. Zanghirati; Complements to the theorem of quasielliptic iterates, Boll. Un. Mat. Ital. A 1 (6) (1982), 137-143. (Reviewer: Gianfranco Bottaro) 35J99 
[68] L. Zanghirati; Iterati di operatori e regolarità Gevrey microlocale anisotropa, Rend. Sem. Mat. Univ. Padova, 67 (1982), 85-104. 